\title{
Mimics of Pramanicin derived from Pyroglutamic acid and their Antibacterial Activity
}

Song Wei Benjamin Tan ${ }^{\mathrm{a}, \mathrm{b}}$, Christina L.L. Chai ${ }^{\mathrm{b}, \mathrm{c} *}$, Mark G. Moloney ${ }^{\mathrm{a} *}$

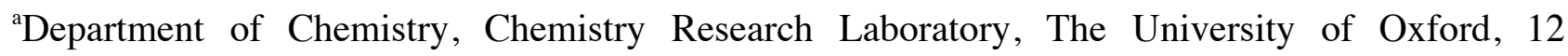
Mansfield Road, Oxford. OX1 3TA.

${ }^{\mathrm{b}}$ Institute of Chemical and Engineering Sciences (ICES), 8 Biomedical Grove, Neuros Building, \#07-01/02/03, Singapore 138665;

${ }^{\mathrm{c}}$ Department of Pharmacy, National University of Singapore, 18 Science Drive 4, Singapore, 117543

mark.moloney@chem.ox.ac.uk

christina_chai@ices.a-star.edu.sg

\begin{abstract}
Mono and dihydroxypyrrolidinones are readily available by direct oxygenation of a pyroglutamate-derived bicyclic lactam with high diastereoselectivity, and these may be manipulated further in protected or unprotected form by Grignard addition to a pendant Weinreb amide to give acylhydroxypyrrolidinones, which are analogues of the natural product, pramanicin. Preliminary bioassay against $S$. aureus and E. coli indicated that some compounds exhibit selective Gramnegative antibacterial activity, and may offer promise for the development of novel systems suitable for antibacterial drug development.
\end{abstract}

The critical importance of natural products in the development of pharmaceutically active compounds has been thoroughly documented, and although popularity of this approach has waned in recent years in favour of combinatorial and rational design, there have been strong calls for its reinvigoration..$^{1-4}$ These calls are particularly relevant for antibacterials, for which there is a serious 
deficit of new candidates in the drug pipeline, ${ }^{5-8}$ at a time when there is considerable urgency to expand therapeutics as a result of the rapid emergence of resistant bacterial strains. ${ }^{9-16}$ The challenges peculiar to antibacterial drug discovery ${ }^{17-25}$ mean that natural products often provide biologically validated start points suitable for immediate elaboration in the quest for new pharmaceutically useful agents. We have recently shown that small molecule mimics of streptolydigin $^{26}$ and kibdelomycin ${ }^{27}$ offer promise in that regard, ${ }^{28-30}$ and we became interested in the exploration of pramanicin as a model start point using a similar strategy. Pramanicin $\mathbf{1}$ was isolated in 1994 and shown to exhibit antifungal activity and antibacterial activity, ${ }^{31}$ but with significant human cytotoxicity. ${ }^{32}$ We recently reported that epoxy analogues were readily available by elaboration of a pyroglutamate substrate, and that the derived compound libraries exhibited antibacterial activity against both Gram-positive and -negative bacteria. ${ }^{33}$ We report here an extension of this work to include monohydroxy and dihydroxy derivatives, which complements the direct synthesis of the cis-dihydroxyl unit which has recently been reported, ${ }^{34}$ providing access to libraries which exhibit antibacterial activity against S. aureus and/or E. coli.

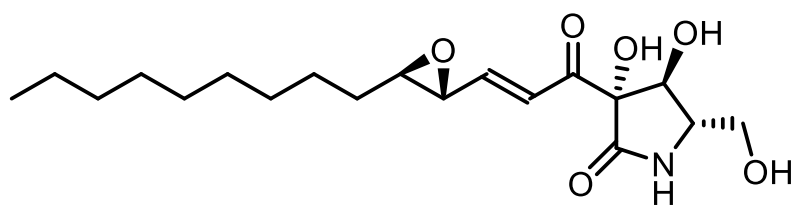

Pramanicin 1

\section{Results and Discussion}

Our approach for access to such hydroxyl systems began with ester $\mathbf{2}$, itself readily derived from pyroglutamic acid, ${ }^{33}$ and the two diastereomers of the hydroxyl product 3a were obtained using the procedure of Christoffers ${ }^{35}$ and isolated separately in yields of $13 \%(7 S)$ and $28 \%(7 R)$. The stereochemistry of the hydroxyl-ester (7R)-3a was determined using nOeSY (Figure 1), and the (7S)-3a isomer was assigned via the analogous chemical shifts pattern of the H-6 protons known to be distinctive for such lactam scaffolds. ${ }^{36}$ In addition, $\alpha$-chlorinated $\mathbf{3 b}$ was isolated in lower yield from this reaction as a mixture of inseparable diastereomers. Given the low isolated yield of the desired product $\mathbf{3 a}$, an alternative hydroxylation approach was required. 

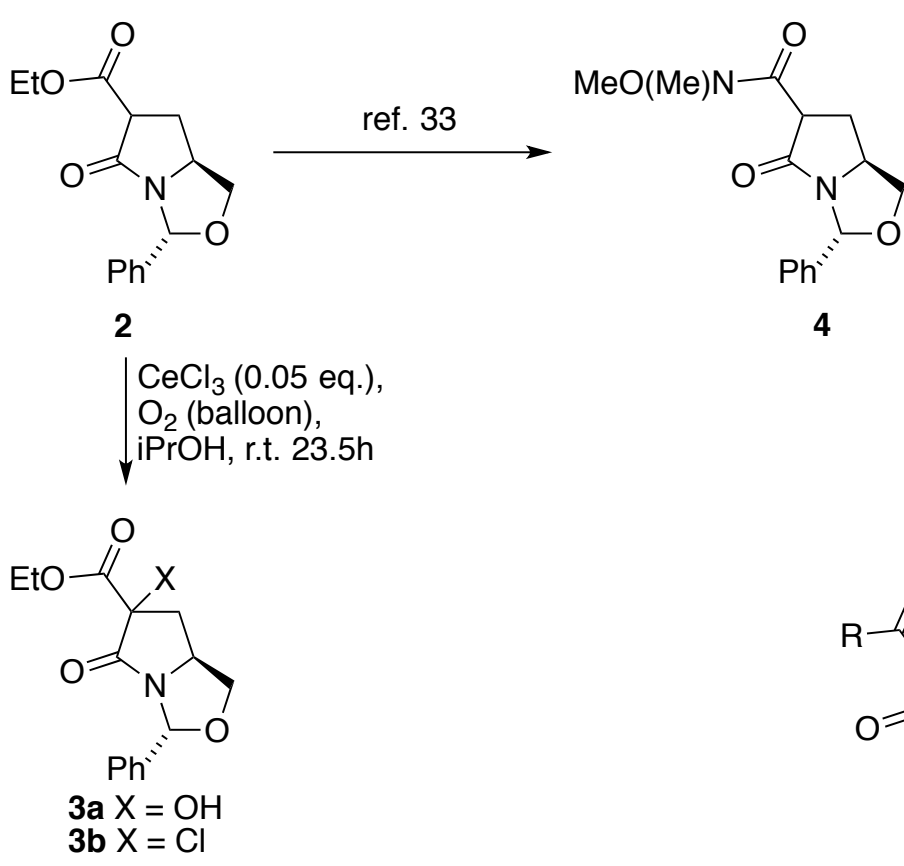

KHMDS (1.3 eq), Oxaziridine $5(1.0 \mathrm{eq}), \mathrm{MeO}(\mathrm{Me}) \mathrm{N}-\mathrm{CH}_{7} \mathrm{OH}$
$\mathrm{THF},-78^{\circ} \mathrm{C}$

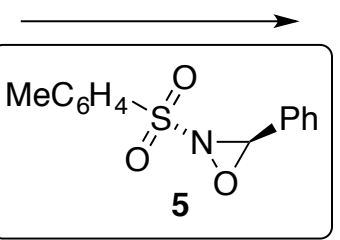

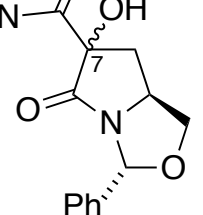

6<smiles>[R]C(=O)[C@]1(O)C[C@@H](CO)NC1=O</smiles>

8a-g

Scheme 1

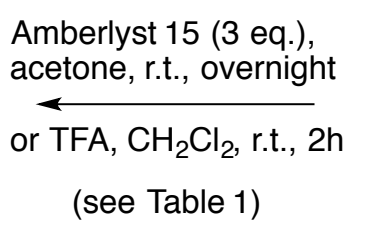

(see Table 1)
$\operatorname{RMgBr}(2.2$ eq.) $\mathrm{THF}, 0^{\circ} \mathrm{C}$

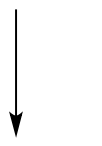

7a-g

We therefore turned to the reported Weinreb amide $\mathbf{4}$, readily prepared from ester $\mathbf{2},{ }^{33}$ which was deprotonated with KHMDS and oxygenated with oxaziridine 5 to give hydroxyl derivative $\mathbf{6}$ in $57 \%$ yield and a diastereomeric ratio of 5.6:1 in favour of the $7 R$ exo diastereomer (Scheme 1), for which the stereochemistry was again assigned from the chemical shift and splitting of the two H-6 signals in the ${ }^{1} \mathrm{H}$ NMR spectrum. ${ }^{33,36}$ Amide 6 was successfully reacted with 2.2 eq. Grignard reagent to give the respective ketones $\mathbf{7 a - g}$ in variable yields, even in the presence of the unprotected tertiary alcohol (Scheme 1 and Table 1); the selectivity of this process in such a functionally dense substrate is remarkable, and offers an alternative approach for the introduction of acyl residues to our recently published protocol based upon carbodiimide coupling of tetramates with carboxylates. ${ }^{37}$ The efficiency of this conversion was found to be dependent upon the stereochemistry of lactam $\mathbf{6}$ at C-7, with (7S)-6 being less efficient than (7R)-6 (note that the $R, S$ assignment changes due to the priority rule change upon conversion to the ketone 7) probably as a result of greater steric hindrance in this endo- system. Both diastereomers of each of 7a-g could be separated by chromatography, and the relative stereochemistry of $\mathbf{7 b}$ was determined via NOESY analysis (Figure 1). As has been previously observed with this class of bicyclic lactam, ${ }^{33,36,38}$ the chemical shift difference between the two H-6 protons also allows ready distinction of the 
diastereomers, since when the tertiary C-7 hydroxyl group is at the endo position of the bicyclic system, the chemical shift difference between the two H-6 protons is significantly greater than the chemical shift difference between the two H-6 protons when the C-7 hydroxyl group is in the exo position. Although it has been noted that the bicyclic system generally exhibits exostereoselectivity in its reactions on the less hindered convex face as seen in this case and as might be expected, ${ }^{39}$ it is nonetheless possible to favour endo-selectivity in the alkylations of the lactam enolates by use of acetophenone protected systems. ${ }^{40,41}$ Overall, conversion of (7S)-6 is best with alkynyl Grignard reagents, followed by aryl and alkyl Grignard reagents, with the bulky isobutyl Grignard reagent giving the lowest conversion, suggesting that the steric requirements in the vicinity of the endo-Weinreb amide dictate the course of the reaction, as might be expected. In order to probe this behaviour further, a competition reaction of $\mathbf{6}$ using phenylethnyl magnesium bromide and isobutyl magnesium bromide added simultaneously was conducted. No isobutyl product was obtained, and the ratio of $(7 S)-7 \mathbf{f}($ exo-OH) to $(7 R)-7 \mathbf{f}($ endo-OH) was 5:1, while the ratio of total ynone $\mathbf{7 f}$ to recovered starting $\mathbf{6}$ was 10:1, suggesting good overall conversion to the ketone product. This outcome confirms that bulky Grignard reagents react more slowly with Weinreb amide 6.

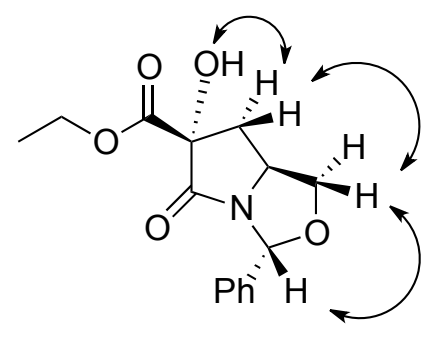

$(7 R)-3 \mathbf{a}$

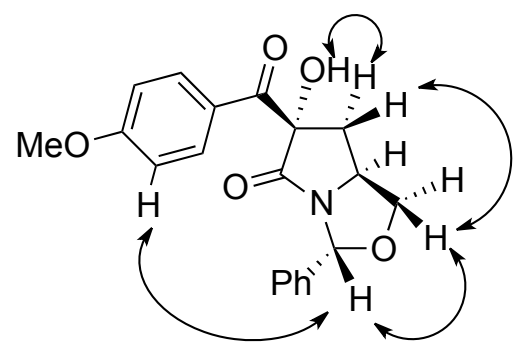

(7S)-7b

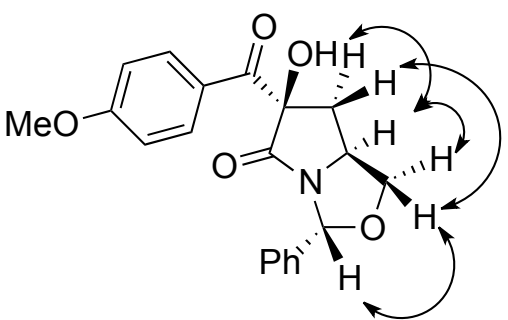

$(7 R)-7 \mathrm{~b}$

Figure 1

Products (7S)-7a-g could be deprotected under mildly acidic conditions (with Amberlyst 15) to give high yields of pyroglutaminols (2S)-8a-g, and alternative hydrolysis conditions (TFA 20 eq., $\mathrm{CH}_{2} \mathrm{Cl}_{2}$, r.t., $2 \mathrm{~h}$ ) also gave good yields of (2S)-8f,g. However, of interest was that hydrolysis of the alternative diastereomer (7R)-7a with Amberlyst 15 (3 eq.) at r.t. overnight gave no conversion and starting material was recovered quantitatively, while hydrolysis under standard conditions with 
excess TFA in $\mathrm{THF} / \mathrm{H}_{2} \mathrm{O}$ gave a complex crude mixture. Overall, the Weinreb amide provided ready access to libraries of monohydroxyl pyroglutaminols.

Table 1: Yields for the Grignard reaction of lactam $\mathbf{6}$ giving products $\mathbf{7}$ and $\mathbf{8}$.

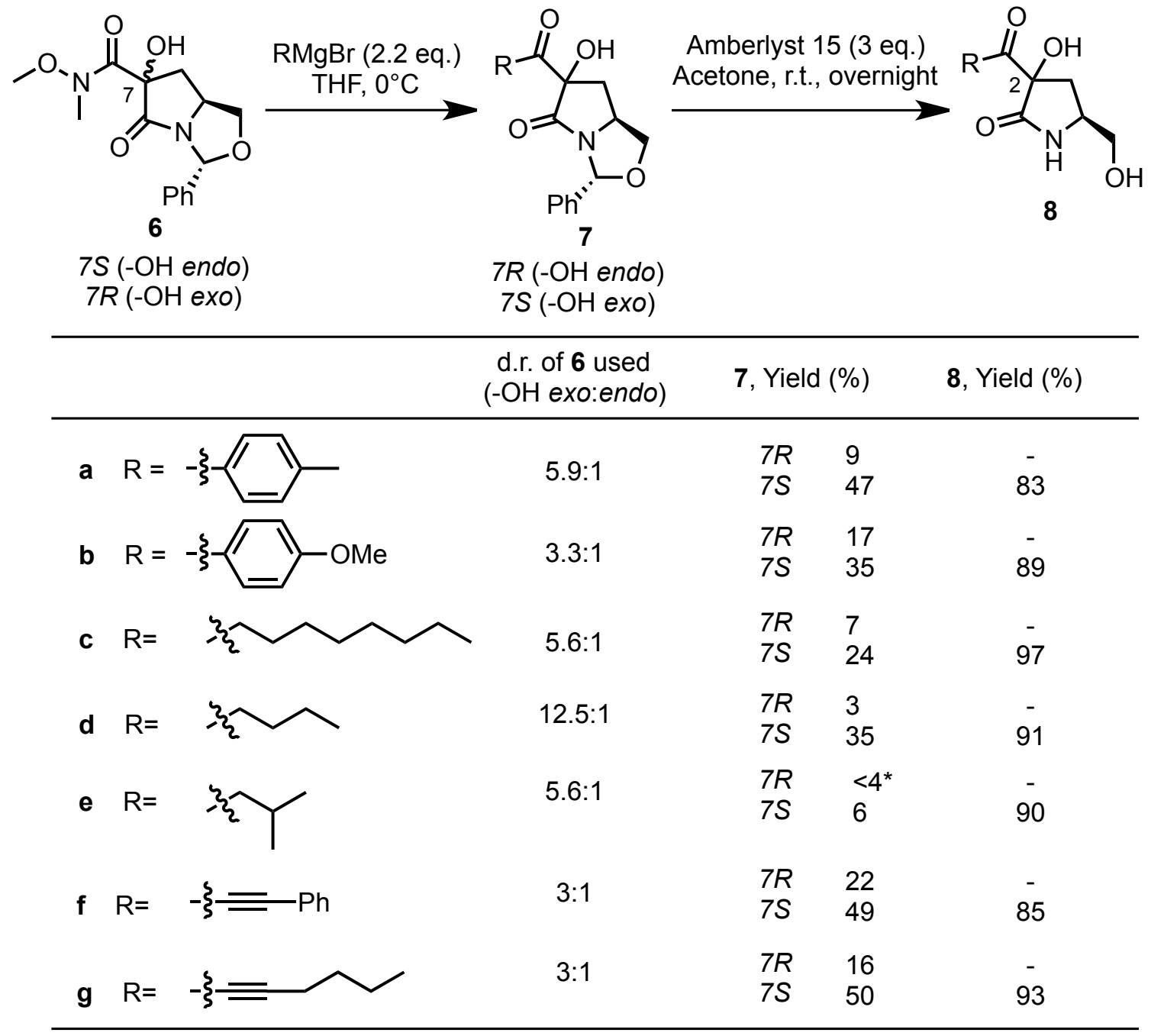

*Isolated with impurities.

Lactam 4 may also be selenylated and eliminated to give the unsaturated derivative $9 ;^{33}$ since such $\alpha, \beta$-unsaturated lactams ${ }^{42}$ are quite electrophilic and suitable for a variety of nucleophilic addition processes, ${ }^{43,}{ }^{44}$ and our earlier work had demonstrated efficient epoxidation of these systems, ${ }^{33}$ an obvious question was its applicability to dihydroxylation processes, which in any case would provide a closer set of mimics to pramanicin 1. However, although an earlier examination of a closely related ester had indicated successful dihydroxylation using osmium tetroxide or potassium permanganate was possible but that product isolation was problematic, application of the 
ruthenium-mediated "flash dihydroxylation" procedure reported by Shing and co-workers ${ }^{45}$ to 9 gave the desired cis-exo-diol 10 with an isolated yield of $69 \%$ (along with some cis-endo-diol 10, d.r. of 5:1, as an inseparable mixture) as determined from the ${ }^{1} \mathrm{H}$ NMR spectrum (based on the corresponding H-2 resonances) (Scheme 2). A NOESY experiment suggested that the exo-diol was formed (Figure 2), and from nOe correlations between the lactam -OMe group with $\mathrm{H}-2$ and $\mathrm{H}$ $4 e n d o$; that the Weinreb amide moiety was on the endo face of the bicycle was confirmed by X-ray crystal structural analysis (Figure 3). ${ }^{46}$ This outcome was in agreement with the stereoselectivity observed with such bicyclic [3.3.0] scaffolds, ${ }^{43,44,47}$ although noteworthy is a recent report indicating that endo- addition may be favoured in certain $\mathrm{N}, \mathrm{O}$-hemiaminal ether systems. ${ }^{48}$ In addition, a single diastereomer of tetramic acid $\mathbf{1 1}$ (assigned by single crystal analysis, Figure 3) was also isolated, presumably arising from over-oxidation of $\mathbf{1 0}$ under the reaction conditions. ${ }^{45}$ 


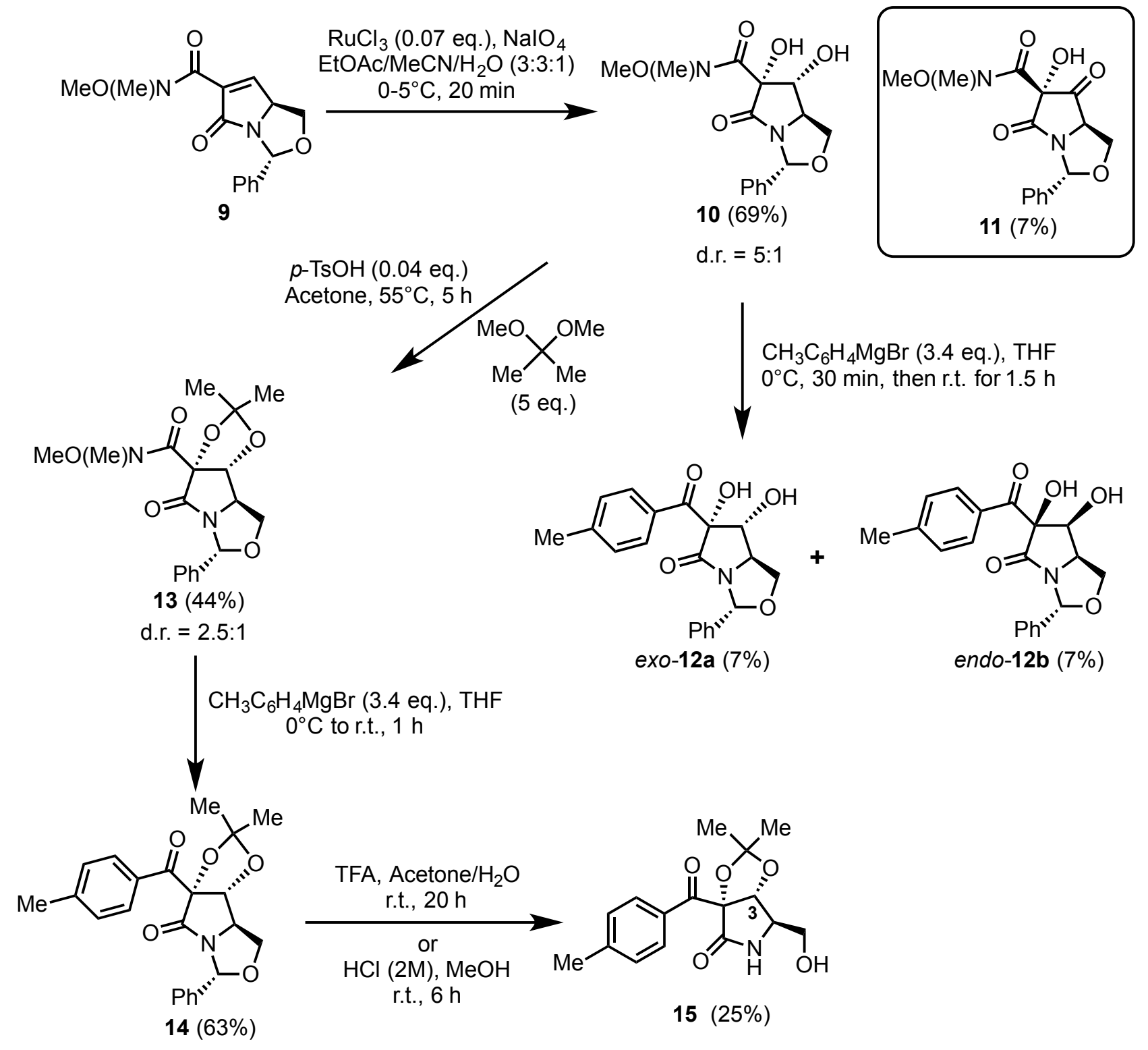

Scheme 2

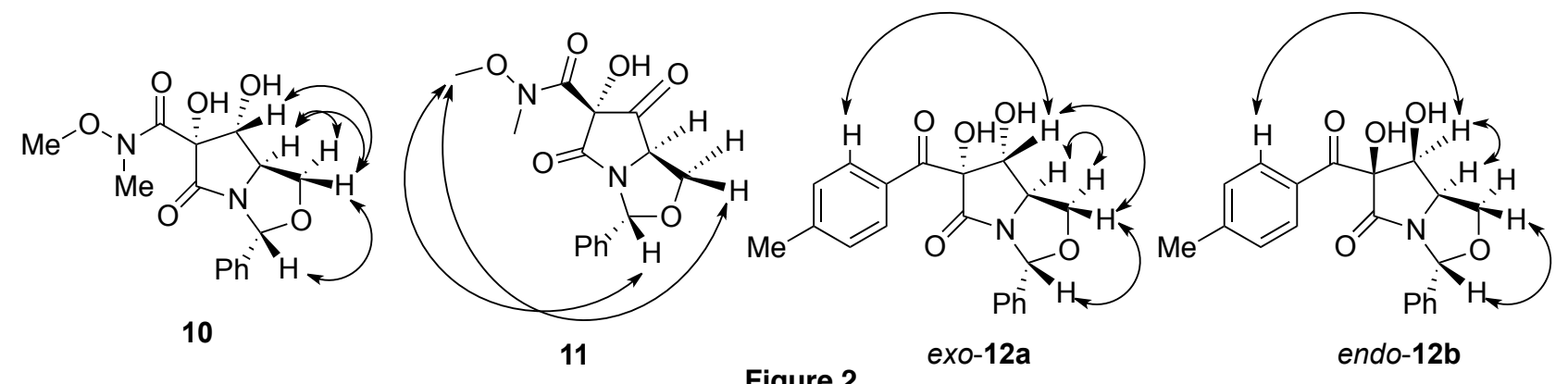

Figure 2 

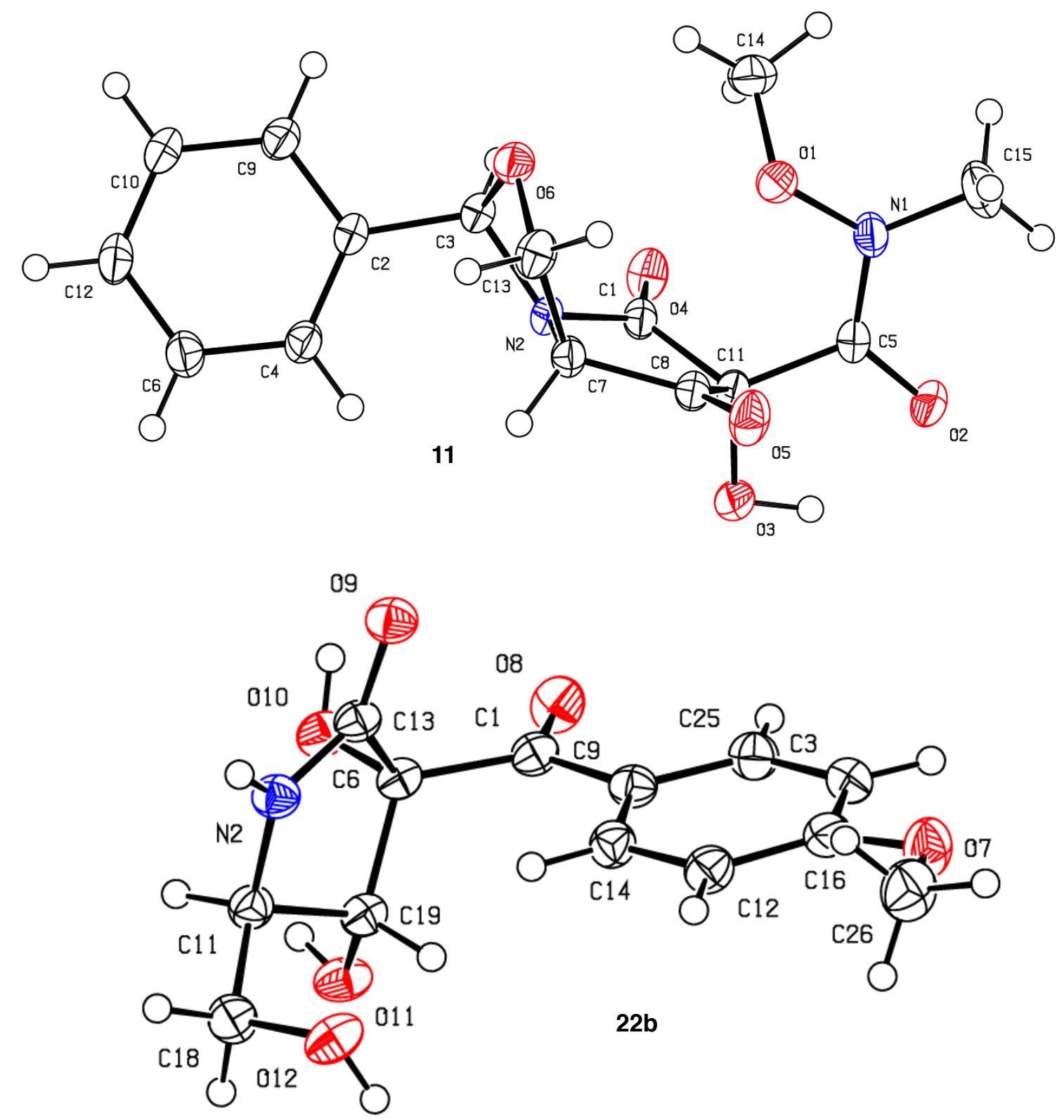

Figure 3: ORTEP plots for structures 11 and 22b

When the Grignard reaction with the Weinreb function using tolylmagnesium bromide was attempted with the unprotected diol 10, a very low conversion to the desired products $\mathbf{1 2 a}, \mathbf{b}$ was obtained (which further confirmed the existence of $\mathbf{1 0}$ as a mixture of cis-exo and cis-endo isomers), in contrast to the reaction of lactam $\mathbf{6}$, and their very similar chromatographic retention factors made purification difficult (Scheme 2); stereochemistry was assigned by NOESY experiment (Figure 2). In order to improve the chemical yield by removing the acidic hydroxylic functionality, the diol was protected as acetonide 13 (44\% yield, major cis-exo and minor cis-endo) under standard conditions, which when followed by Grignard reaction as above gave ketone $\mathbf{1 4}$ in a much 
improved yield of $63 \%$ yield as a mixture of diastereomers (exo diol: endo diol 2.5:1). Although a single spot was observed by TLC analysis of $\mathbf{1 4}$, its ${ }^{1} \mathrm{H}$ NMR spectrum contained two sets of H-6 signals, with some of the other signals being broad. A variable temperature ${ }^{1} \mathrm{H}$ and ${ }^{13} \mathrm{C}$ NMR experiment at $65^{\circ} \mathrm{C}(338 \mathrm{~K})$ improved the resolution of the broad peaks, but the ratio of these two forms remained essentially unchanged, suggesting their identity as diastereomers and not rotamers, thereby confirming the formation of cis-exo and cis-endo products, but as an inseparable mixture. However, various acidic hydrolysis conditions failed to simultaneously deprotect both the oxazolidine and the acetonide functions, and strong acidic conditions (2 $\mathrm{M}$ aq. $\mathrm{HCl}$ (4 eq.)) $\mathrm{MeOH}$, r.t., $6 \mathrm{~h}$ gave the cis-exo diastereomer of $\mathbf{1 5}$ in $25 \%$ yield.

Given this difficult deprotection, other more acid-labile diol protecting groups were investigated, beginning with a benzylideneacetal protecting group (Scheme 3), notwithstanding the possible complications resulting from the new stereogenic centre. Acetal $\mathbf{1 6}$ (as a mixture of diastereomers) was readily prepared in good yield and elaborated to ketone $\mathbf{1 7}$ as before. However, again, on attempted hydrolysis with TFA, only the oxazolidine was hydrolysed (Scheme 3) and after purification, both 18 and unreacted $\mathbf{1 7}$ were isolated each as a single diastereomer, in 13 and $16 \%$ yield respectively. The relative stereochemistry of recovered $\mathbf{1 7}$ was readily determined via NOESY (Figure 4). For 18, NOESY gave correlations between H6-NH, H6-H4, H3-H4 but this data was insufficient to assign the stereochemistry of the protected diol relative to the pyrrolidinone. In order to establish this, MM2-minimised molecular modelling was used to generate the most stable conformers of all four possible diastereomers of 18, and this suggested that only one diastereomer could have the H-6 oriented in close proximity to both $-\mathrm{NH}-$ and $\mathrm{H}-4$, enabling its assignment as shown in Scheme 3 and Figure 4.

Given the stability of the benzylidene acetal towards Bronsted acid, deprotection under reductive conditions was investigated. When a mixture of diastereomers of $\mathbf{1 8}$ was treated with hydrogen gas in the presence of $\mathrm{Pd} / \mathrm{C}$ catalyst, reduction of the ketone to the hydroxyl occurred, rather than hydrogenolysis, giving pyroglutaminol 19 in $31 \%$ yield as a single diastereomer. The 
stereochemistry of the protected diol, benzylidene and newly formed hydroxyl group were assigned using nOe correlations (Figure 4), and this outcome would appear to arise from least hindered attack by hydrogen, again as might be expected.
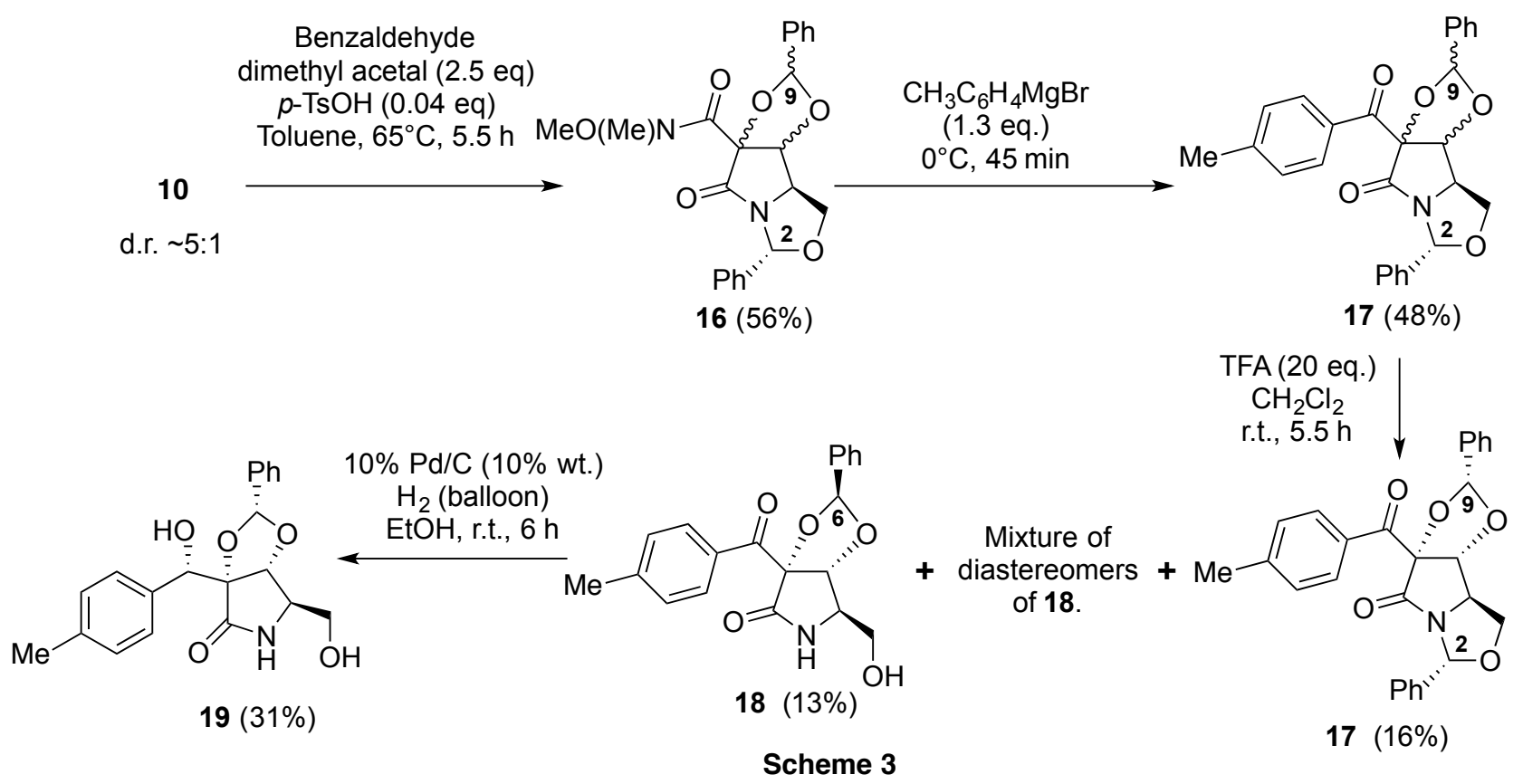

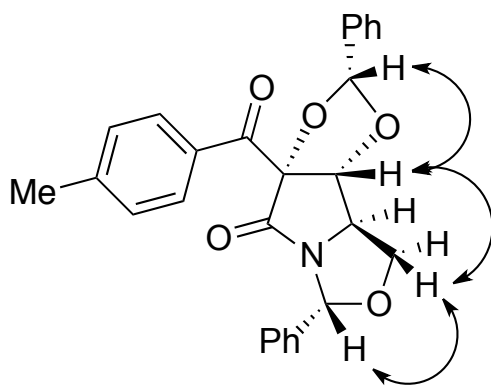

17

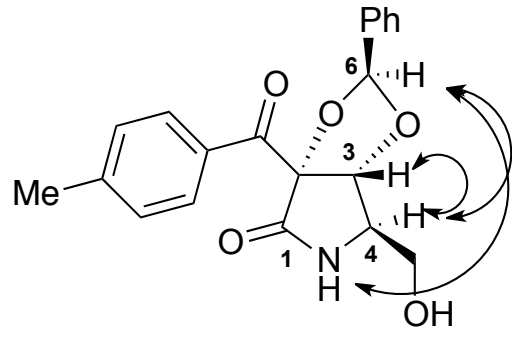

18

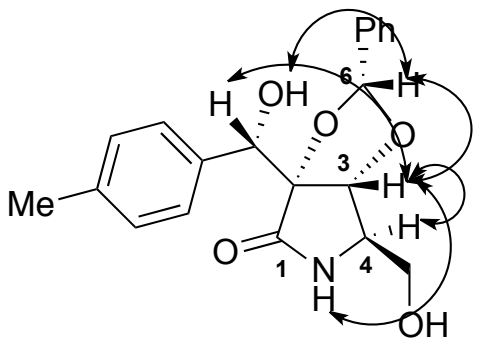

19

Figure 4

Taken together, these results seemed to indicate that acetals in this system might be more stable to acidic hydrolysis than expected. For this reason, the more acid-labile $p$-methoxyphenyl

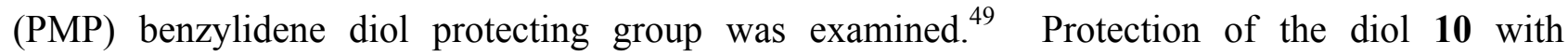
anisaldehyde dimethylacetal gave $\mathbf{2 0}$ in $67 \%$ yield, again as a mixture of diastereomers (Scheme 4). When 20 was reacted with the respective Grignard reagent, the ketones 21a-g were obtained in modest yield (Table 2); this may again be attributed to the steric bulk in the vicinity of the Weinreb amide due to the PMP benzylidene acetal. Treatment with TFA/ $\mathrm{CH}_{2} \mathrm{Cl}_{2}$ gave global deprotection and the desired 2,3-dihydroxypyrrolidinones 22a-g were obtained in good yields (Table 2). Some 
of these derivatives were isolated as a mixture of diastereomers (about the diol), and it was observed that the minor diastereomer had a larger $J_{\mathrm{H} 3-\mathrm{H} 4}$ value and that the $\mathrm{H}-3$ signal resonated further downfield compared to the major diastereomer. On the basis of earlier reactions, it was assumed that the major diastereomer was the exo-diol, and in order to confirm this, exo-12a which had been earlier prepared and isolated as a single diastereomer (see Scheme 2) was subjected to standard acidic hydrolysis conditions; the product thus obtained had the same $J_{\mathrm{H} 3-\mathrm{H} 4}$ value of $6.4 \mathrm{~Hz}$ as that of 22a obtained via the PMP protected intermediate, confirming their identity. In addition, X-ray crystallography of $\mathbf{2 2 b}$ prepared via the PMP-protected intermediate confirmed its exostereochemistry (Figure 3). ${ }^{46}$

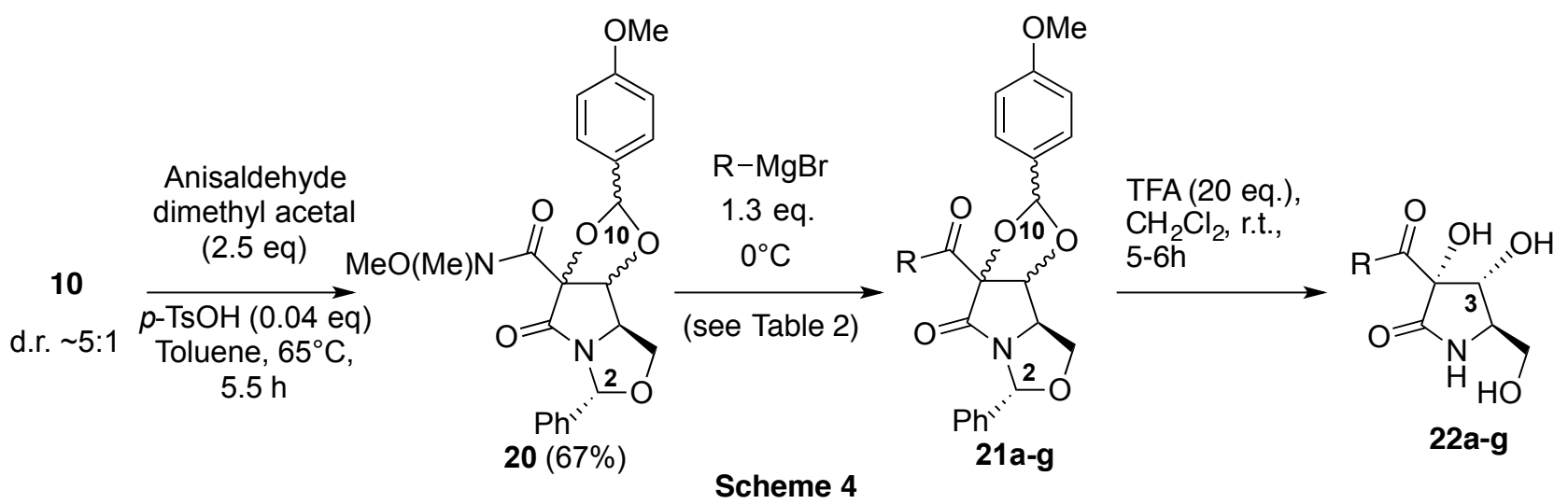


Table 2: Yields of ketones 21 and triols 22.

\begin{tabular}{|c|c|c|c|c|c|}
\hline & & & \multirow[b]{2}{*}{ 21, Yield (\%) } & \multicolumn{2}{|c|}{22} \\
\hline & & & & Yield (\%) & $J_{\mathrm{H} 3-\mathrm{H} 4}$ \\
\hline $\mathbf{a}$ & $R=$ & & 45 & 48 & $6.4 \mathrm{~Hz}$ \\
\hline b & $\mathrm{R}=$ & $-\xi-<$ & 44 & 52 & $6.4 \mathrm{~Hz}$ \\
\hline c & $R=$ & & 30 & 41 ; d.r. $5: 1$ & $\begin{array}{l}6.1 \mathrm{~Hz} \text { (Major) } \\
6.7 \mathrm{~Hz} \text { (Minor) }\end{array}$ \\
\hline d & $R=$ & & 19 & 58; d.r. 2:1 & $\begin{array}{l}6.1 \mathrm{~Hz} \text { (major) } \\
6.8 \mathrm{~Hz} \text { (minor) }\end{array}$ \\
\hline e & $R=$ & & 27 & 31 & $6.1 \mathrm{~Hz}$ \\
\hline f & $R=$ & $-\xi \equiv-P h$ & 51 & 48; d.r. 3.3:1 & $\begin{array}{l}\text { 6.7 Hz (major) } \\
7.0 \mathrm{~Hz} \text { (minor) }\end{array}$ \\
\hline & $R=$ & & $34^{*}$ & $72 ;$ d.r. $1: 1$ & $\begin{array}{l}6.8 \mathrm{~Hz} \text { (major) } \\
7.2 \mathrm{~Hz} \text { (minor) }\end{array}$ \\
\hline
\end{tabular}

${ }^{*}$ OeSY shows exo-diol but $\sim 1: 1$ epimeric mixture about $\mathrm{H}-10$

With the ynones $\mathbf{8 f}, \mathbf{g}$ and $\mathbf{2 2 f}, \mathbf{g}$ in hand, of interest was the synthesis of the corresponding enones; it was expected that a sequence using Lindlar's catalyst/hydrogen for the partial reduction of ynones followed by isomerisation of the cis-enones in the presence of catalytic amount of iodine would provide the trans-enones. ${ }^{50}$ In the event (Scheme 5), the reduction was found to proceed with poor $(E) /(Z)$ selectivity, but isomerization with iodine cleanly gave the $(E)$ isomer with none of the $(Z)$ isomer detectable by NMR analysis, although in low chemical yield over the two steps. No deoxygenated or over-reduced by-products were isolated from this sequence.<smiles>[R]C(=O)[C@]1(O)C(=O)NC(CO)[C@H]1[X]</smiles>

8f $\mathrm{X}=\mathrm{H}, \mathrm{R}=\mathrm{CCPh}$

$8 \mathbf{g X}=\mathrm{H}, \mathrm{R}=\mathrm{CCCH}_{2} \mathrm{CH}_{2} \mathrm{CH}_{2} \mathrm{CH}_{3}$ $22 \mathrm{X}=\mathrm{OH}, \mathrm{R}=\mathrm{CCPh}$ 22g X $=\mathrm{OH}, \mathrm{R}=\mathrm{CCCH}_{2} \mathrm{CH}_{2} \mathrm{CH}_{2} \mathrm{CH}_{3}$ (i) Lindlar's Catalyst, $\mathrm{H}_{2}$

(ii) $I_{2}$ (cat.), EtOAc ambient temperature,<smiles>[R]C(=O)[C@]1(O)C(=O)N[C@@H](CO)[C@H]1[X]</smiles>

23a $\mathrm{X}=\mathrm{H}, \mathrm{R}=(E)-\mathrm{CH}=\mathrm{CHPh}(35 \%)$

23b $\mathrm{X}=\mathrm{H}, \mathrm{R}=(E)-\mathrm{CH}=\mathrm{CHCH}_{2} \mathrm{CH}_{2} \mathrm{CH}_{2} \mathrm{CH}_{3}$ (38\%)

23c $\mathrm{X}=\mathrm{H}, \mathrm{R}=\mathrm{CH}_{2} \mathrm{CH}_{2} \mathrm{CH}_{2} \mathrm{CH}_{2} \mathrm{CH}_{2} \mathrm{CH}_{3}(12 \%)$

23d $\mathrm{X}=\mathrm{OH}, \mathrm{R}=(E)-\mathrm{CH}=\mathrm{CHPh}(16 \%)$

23e $\mathrm{X}=\mathrm{OH}, \mathrm{R}=(E)-\mathrm{CH}=\mathrm{CHCH}_{2} \mathrm{CH}_{2} \mathrm{CH}_{2} \mathrm{CH}_{3}(17 \%)$

\section{Scheme 5}




\section{Antibacterial Activity}

In order to assess whether any members of the above chemical libraries, which might be considered to be mimics of pramanicin 1, exhibited antibacterial activity, assays were performed using the hole-plate method against either Staphylococcus aureus DS267 (Gram-positive) or Escherichia coli X580 (Gram-negative), using Cephalosporin C (CephC) as a standard (Table 3 and Figure 4). ${ }^{51}$ It was found that 2-hydroxypyrrolidinones with a C-7 carboxamido substituent 6 or a C-7 carboxylate substituent 3a did not exhibit antibacterial activity, while 2-chloro-carboxylpyrrolidinone 3b exhibited some activity with E. coli. By contrast, 2-hydroxy-ketopyrrolidinones 7a-f, 8a-i,k generally exhibited antibacterial activity, and with high selectivity towards E.coli over S.aureus. However, lower selectivity was observed for derivatives with Michael acceptor functionalities (for example ynones or enones) on their side-chain, due to their better activity with S.aureus. For some derivatives, the minor hydroxyl diastereomer $((7 R)-7 \mathbf{a}-\mathbf{c},(7 R)-7 \mathbf{f})$ exhibited greater activity towards E.coli as compared to the major hydroxyl diastereomer ((7S)-7a-c,,$(7 R)-\mathbf{7 f})$.

Table 3: Antibacterial Bioactivity and Physicochemical Parameters for Selected Compounds. a,

\begin{tabular}{|c|c|c|c|c|c|c|c|c|}
\hline \multirow[t]{2}{*}{ Compound } & \multirow{2}{*}{$\begin{array}{c}\text { Conc. } \\
(\mathrm{mg} / \mathrm{mL})\end{array}$} & \multicolumn{2}{|c|}{ Zone Size/mm } & \multirow[t]{2}{*}{ MW } & \multirow[t]{2}{*}{$\operatorname{clog} \mathrm{D}_{7.4}$} & \multirow[t]{2}{*}{ PSA } & \multirow[t]{2}{*}{ MSA } & \multirow[t]{2}{*}{$\% \mathrm{PSA}$} \\
\hline & & S. Aureus & E. Coli & & & & & \\
\hline \multicolumn{9}{|c|}{ Monohydroxypyroglutaminols } \\
\hline$(7 S)-3 a$ & 2 & 10 & 10 & 291.3 & 0.95 & 76.07 & 415.03 & 18.3 \\
\hline$(7 R)-3 a$ & 2 & 10 & 10 & 291.3 & 0.95 & 76.07 & 415.03 & 18.3 \\
\hline $\mathbf{3 b}$ & 2 & 10 & $13(\mathrm{H})$ & 309.75 & 2.31 & 55.84 & 419.69 & 13.3 \\
\hline 6 & 2 & 10 & 10 & 306.32 & 0.24 & 79.31 & 434.74 & 18.2 \\
\hline$(7 S)-7 \mathrm{a}$ & 2 & 10 & 15 & 337.38 & 2.72 & 66.84 & 473.47 & 14.1 \\
\hline$(7 R)-7 a$ & 2 & 10 & 17 & 337.38 & 2.72 & 66.84 & 473.47 & 14.1 \\
\hline$(7 S)-8 a$ & 2 & 10 & 10 & 249.27 & -0.03 & 86.63 & 356.52 & 24.3 \\
\hline$(7 S)-7 b$ & 2 & 10 & $13(\mathrm{H})$ & 353.37 & 2.05 & 76.07 & 489.55 & 15.5 \\
\hline$(7 R)-7 b$ & 2 & 10 & $14(\mathrm{H})$ & 353.37 & 2.05 & 76.07 & 489.55 & 15.5 \\
\hline$(7 S)-8 \mathrm{~b}$ & 2 & 10 & $13(\mathrm{H})$ & 265.27 & -0.7 & 95.86 & 372.33 & 25.7 \\
\hline$(7 S)-7 c$ & 2 & 10 & 14 & 359.47 & 4.15 & 66.84 & 581.86 & 11.5 \\
\hline$(7 R)-7 \mathrm{c}$ & 2 & 10 & 17 & 359.47 & 4.15 & 66.84 & 581.86 & 11.5 \\
\hline$(7 S)-8 \mathrm{c}$ & 2 & 10 & 17 & 271.36 & 1.4 & 86.63 & 465.38 & 18.6 \\
\hline$(7 S)-7 \mathrm{~d}$ & 2 & 10 & $13(\mathrm{H})$ & 303.36 & 2.37 & 66.84 & 459.34 & 14.6 \\
\hline$(7 R)-7 d$ & 2 & 10 & 13 & 303.36 & 2.37 & 66.84 & 459.34 & 14.6 \\
\hline$(7 S)-8 \mathrm{~d}$ & 2 & 10 & 10 & 215.25 & -0.38 & 86.63 & 343.14 & 25.2 \\
\hline$(7 S)-7 \mathrm{e}$ & 2 & 10 & $13(\mathrm{H})$ & 303.36 & 2.21 & 66.84 & 458.66 & 14.6 \\
\hline$(7 S)-7 f$ & 2 & 10 & $15(\mathrm{H})$ & 347.37 & 3.16 & 66.84 & 460.85 & 14.5 \\
\hline
\end{tabular}




\begin{tabular}{|c|c|c|c|c|c|c|c|c|}
\hline$(7 R)-7 f$ & 2 & $14(\mathrm{H})$ & 17 & 347.37 & 3.16 & 66.84 & 460.85 & 14.5 \\
\hline$(7 S)-8 f$ & 2 & $15(\mathrm{H})$ & 23 & 259.26 & 0.41 & 86.63 & 344.82 & 25.1 \\
\hline$(7 S)-7 \mathrm{~g}$ & 2 & $13(\mathrm{H})$ & $19(\mathrm{H})$ & 327.38 & 3.35 & 66.84 & 477.76 & 14.0 \\
\hline$(7 R)-7 \mathrm{~g}$ & 2 & $13(\mathrm{H})$ & 16 & 327.38 & 3.35 & 66.84 & 477.76 & 14.0 \\
\hline$(7 S)-8 \mathrm{~g}$ & 2 & $13(\mathrm{H})$ & $23(\mathrm{H})$ & 239.27 & 0.61 & 86.63 & 361.88 & 23.9 \\
\hline$(7 S)-8 \mathrm{~h}$ & 2 & 15 & $20(\mathrm{H})$ & 261.28 & 0.39 & 86.63 & 357.08 & 24.3 \\
\hline$(7 S)-23 b$ & 2 & 15 & 19 & 241.29 & 0.51 & 86.63 & 374.28 & 23.1 \\
\hline$(7 S)-23 \mathrm{c}$ & 2 & 10 & 10 & 243.3 & 0.51 & 86.63 & 404.93 & 21.4 \\
\hline \multicolumn{9}{|c|}{ Dihydroxypyroglutaminols } \\
\hline 10 & 2 & 10 & 10 & 322.32 & -0.45 & 99.54 & 442.29 & 22.5 \\
\hline 13 & 2 & 10 & 10 & 362.38 & 1.25 & 77.54 & 524.64 & 14.8 \\
\hline 14 & 2 & 10 & 10 & 393.44 & 3.73 & 65.07 & 563.65 & 11.5 \\
\hline 15 & 2 & 10 & 10 & 305.33 & 0.98 & 84.86 & 447.05 & 19.0 \\
\hline exo-12a & 2 & $14(\mathrm{H})$ & $20(\mathrm{H})$ & 353.37 & 2.03 & 87.07 & 481.29 & 18.1 \\
\hline endo-12a & 2 & $15(\mathrm{H})$ & $19(\mathrm{H})$ & 353.37 & 2.03 & 87.07 & 481.29 & 18.1 \\
\hline 17 & 2 & 10 & 10 & 441.48 & 5.19 & 65.07 & 601.88 & 10.8 \\
\hline 18 & 2 & 10 & 17 & 353.37 & 2.45 & 84.86 & 484.25 & 17.5 \\
\hline 19 & 1 & 10 & 10 & 355.39 & 2.45 & 84.86 & 485.7 & 17.5 \\
\hline $22 a$ & 2 & 10 & 10 & 265.27 & -0.72 & 106.86 & 364.45 & 29.3 \\
\hline $22 b$ & 2 & 10 & 10 & 281.26 & -1.39 & 116.09 & 380.84 & 30.5 \\
\hline $22 c$ & 2 & 10 & 22 & 287.36 & 0.71 & 106.86 & 474 & 22.5 \\
\hline 22d & 2 & 10 & 10 & 231.25 & -1.07 & 106.86 & 350.48 & 30.5 \\
\hline $22 e$ & 2 & 10 & 10 & 231.25 & -1.22 & 106.86 & 348.4 & 30.7 \\
\hline $22 f$ & 2 & 10 & $13(\mathrm{H})$ & 275.26 & -0.28 & 106.86 & 352.34 & 30.3 \\
\hline $22 \mathrm{~g}$ & 2 & 10 & 10 & 255.27 & -0.08 & 106.86 & 368.82 & 29.0 \\
\hline 23d & 2 & 10 & $14(\mathrm{H})$ & 277.28 & -0.3 & 106.86 & 364.8 & 29.3 \\
\hline $23 e$ & 2 & 15 & 15 & 257.29 & -0.18 & 106.86 & 382.12 & 28.0 \\
\hline
\end{tabular}

a $(\mathrm{H})$ - represents that the zone exists as a "halo", where an area of reduced bacteria density was observed instead of a distinct clear zone; ${ }^{b}$ For a concise summary of structures relevant to this table, see Figure (ESI).

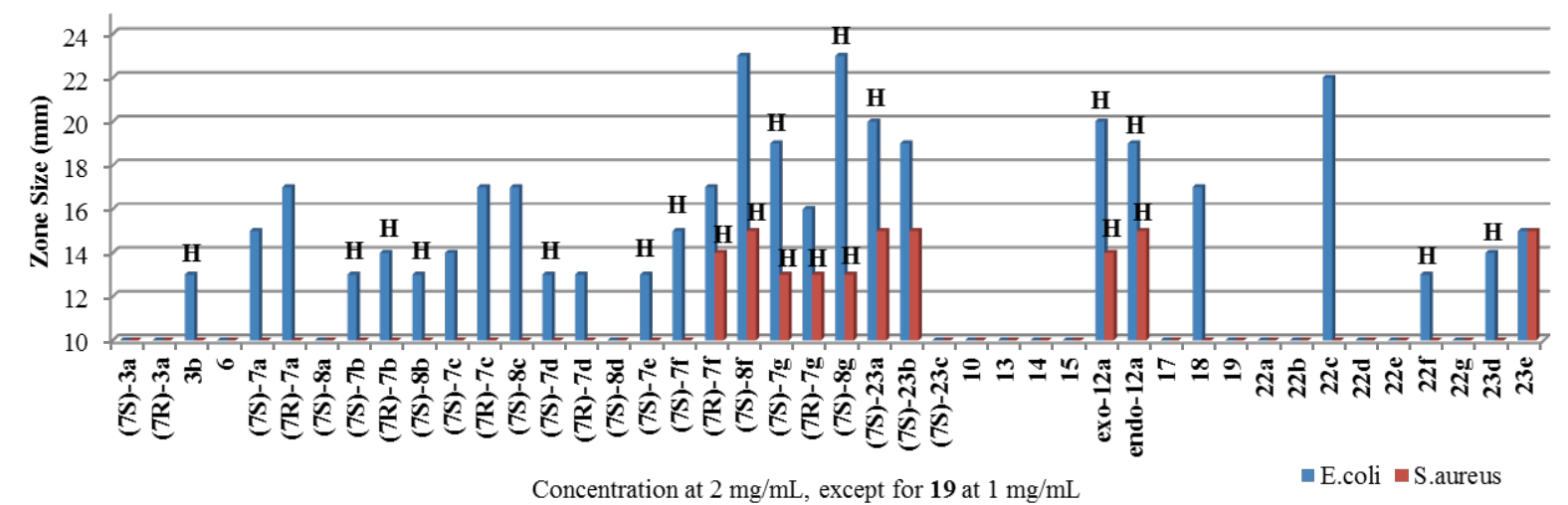

Figure 5: Bioactivity of monohydroxy and dihydroxypyroglutaminols

Analysis of the bioactivity of protected 2,3-dihydroxypyrrolidinones suggested that the exocyclic ketone at C-7, together with the presence of the free hydroxyl groups at C-6 and C- 7, were important for activity (Figure 4 and Table 5). Absence of the oxazolidine group (compare 15 with 14) did not alter 
the antibacterial activity, but absence of the acetonide (compare 14 with 12a) resulted in a significant increase in antibacterial activity. From a comparison of 15 with 14, 17 might have been expected to have similar antibacterial activity after oxazolidine hydrolysis to $\mathbf{1 8}$. However, $\mathbf{1 8}$ was observed to have significant activity with $E$. coli, perhaps due to the different stereochemistry of the benzylidene group. Interestingly, 2,3-dihydroxypyrrolidinones 22a-i, all possessing a scaffold that is most structurally similar to pramanicin, did not show strong antibacterial effects. Only four out of the nine derivatives exhibited any antibacterial activity, with good antibacterial activity towards $E$. coli being observed for 22c. These results suggest that either a long hydrophobic side-chain (22c) or one that contains a Michael acceptor (22f, 22g-h) plays an important role in the observation of antibacterial activity.

Optimal bioactivity for the different molecular scaffolds correlated with their respective clogP and \%PSA ranges, and the data are summarised in Table 4 and Figure $5 .{ }^{52}$ It was observed that compounds active towards $E$. coli only are more polar than compounds active towards $S$. aureus only, as shown by lower clogD7.4 or higher \%PSA values, while compounds active towards both E. coli and S. aureus generally have intermediate clogD7.4 and \%PSA values, and this is likely to be related to their ability to penetrate the bacterial cell wall. ${ }^{53}$ Antibacterial drugs are generally more polar than other drugs, and a certain degree of polarity is required for bactericidal molecules as the cell walls of both Gram-positive and Gram-negative bacteria are charged. ${ }^{54,55}$ This contrasts with Lipinski's "rule of five" (good absorption predicted if $\mathrm{H}$-bond donors $<5$; $\mathrm{H}$-bond acceptors $<10$; molecular weight $<500$; $\operatorname{clogP}<5$ ) which is applicable for the prediction of good oral absorption in mammalian systems. ${ }^{56}$

Table 4: cLogP and \%PSA ranges of compounds with optimal bioactivity against S. aureus and E. coli.

\begin{tabular}{|c|c|c|c|c|c|c|}
\hline Molecular Scaffold & \multicolumn{3}{|c|}{ S. aureus activity } & \multicolumn{3}{c|}{ E. coli activity } \\
\cline { 2 - 7 } & $\begin{array}{c}\text { Zone size } \\
\text { range/mm }\end{array}$ & $\begin{array}{c}\text { clogP } \\
\text { range }\end{array}$ & $\begin{array}{c}\text { \%PSA } \\
\text { range }\end{array}$ & $\begin{array}{c}\text { Zone size } \\
\text { range }\end{array}$ & $\begin{array}{c}\text { clogP } \\
\text { range }\end{array}$ & $\begin{array}{c}\text { \%PSA } \\
\text { range }\end{array}$ \\
\hline 2-Hydroxypyrrolidinones 7 & 13 & 3.16 & 14.5 & $13-16$ & $2.05-4.15$ & $11.5-15.5$ \\
\hline $\begin{array}{c}\text { 2-Hydroxypyrrolidinones 8 } \\
\text { 2,3-Dihydroxypyrrolidinones } \\
\mathbf{2 2} \text { and 23 }\end{array}$ & Nil & - & - & $14-20$ & $0.41-1.4$ & $18.6-25.1$ \\
\hline
\end{tabular}




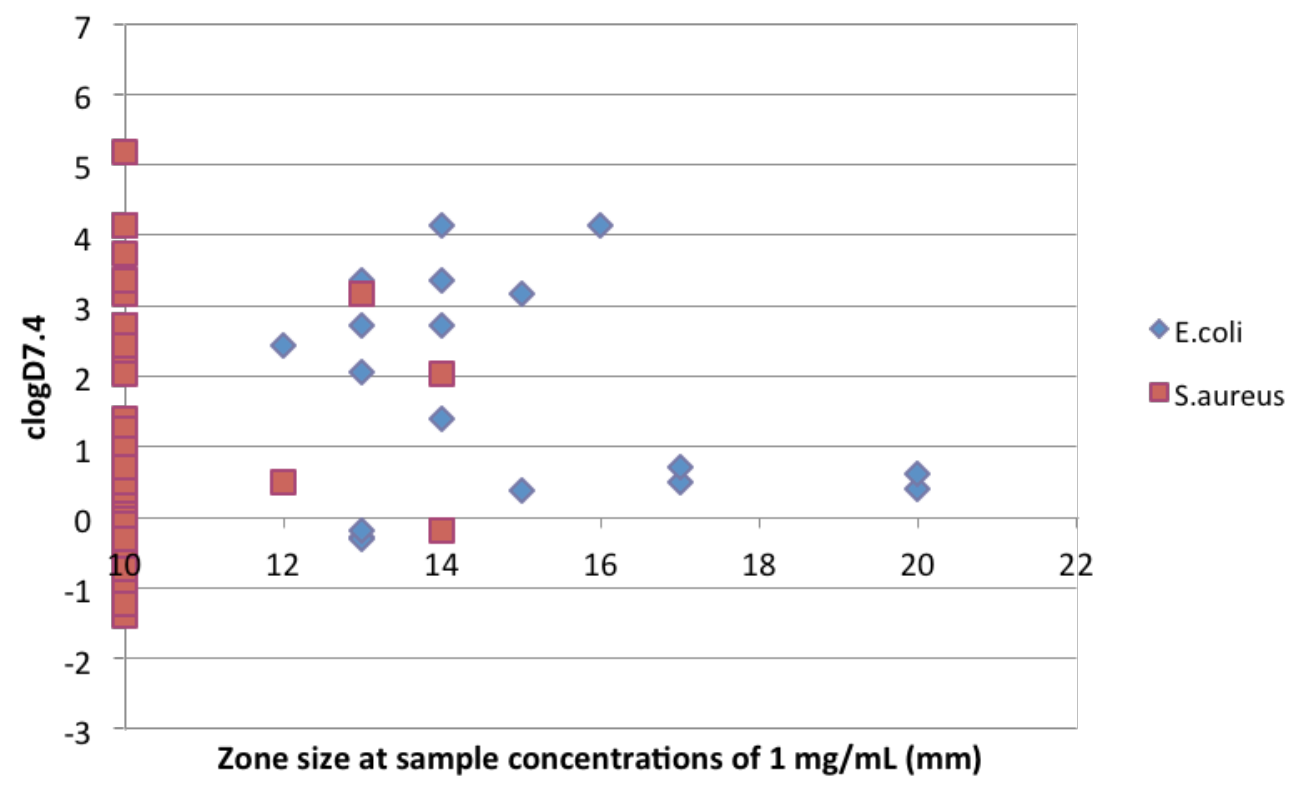

Figure 5. Correlation of $\operatorname{cog} \mathrm{D}_{7.4}$ (polarity) with the zone sizes (antibacterial activity) of pyroglutamate-derived.

\section{Conclusion}

Mild and facile hydroxylation conditions have been identified, which along with a protocol exploiting the Grignard addition to a Weinreb amide, permits the direct and rapid construction of mono- and dihydroxypyrrolidinones from a pyroglutamate scaffold. An assessment of their antibacterial properties showed that the compounds most active against $E$. coli are those with higher polarity (cLogP values $<1$ and $\% \mathrm{PSA}>22)$ probably as a result of their greater bacterial cell wall permeability. ${ }^{57}$ However, it is clear that appropriate ring functionalisation is critically important for antibacterial activity, especially since unsubstituted systems show little activity. ${ }^{58}$ This work shows that pyroglutamates can provide useful structurally well-defined 3D building blocks with large Fsp3 values, ${ }^{59,60}$ generating novel structures suitable for inclusion in the antibacterial drug discovery process. $^{61-63}$

\section{Experimental}

\section{Ce(III)-mediated $\alpha$-hydroxylation of 2}


Lactam 2 (50 mg, $0.182 \mathrm{mmol}, 1.0$ eq.) was added to a solution of $\mathrm{CeCl}_{3} .7 \mathrm{H}_{2} \mathrm{O}(3.4 \mathrm{mg}, 0.009$ mmol, 0.05 eq.) in ${ }^{i} \mathrm{PrOH}(1 \mathrm{~mL})$. The reaction mixture was first bubbled through with $\mathrm{O}_{2}$ (balloon) to saturate the solvent, and left to stir with $\mathrm{O}_{2}$ balloon attached. The reaction was stirred at r.t. for $25 \mathrm{~h}$, and filtered over a pad of silica gel followed by washing with EtOAc. The solvent was evaporated to give the crude which was purified on via silica gel flash column chromatography (Eluent: $10 \%$ to $20 \% \mathrm{EtOAc} / \mathrm{PE}$ ) to give the following:

$(2 R, 5 S, 7 R)$-7-Ethoxycarbonyl-7-hydroxy-8-oxo-2-phenyl-1-aza-3-oxa-bicyclo[3.3.0]octane, (7R)-3a

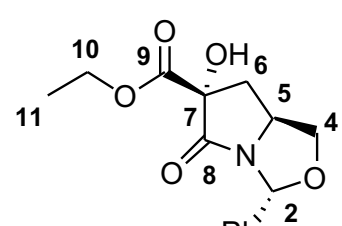

$\mathrm{R}_{f}=0.35$ (1:1 EtOAc:PE); colourless oil; $[\alpha]_{\mathrm{D}}^{23}=+128.8(c=0.5$ in $\mathrm{MeCN})$; $v_{\max } / \mathrm{cm}^{-1}\left(\right.$ film) $3422(\mathrm{br}),, 1744(\mathrm{~s}, \mathrm{C}=\mathrm{O}), 1711(\mathrm{~s}, \mathrm{C}=\mathrm{O}) ; \delta_{\mathrm{H}}(400 \mathrm{MHz}$, $\left.\mathrm{Ph}^{2} \mathrm{CDCl}_{3}\right)$ 7.45-7.48 (m, 2H, PhH), 7.33-7.40 (m, 3H, PhH), 6.29 (s, 1H, H-2), 4.34 (q, $J=7.1 \mathrm{~Hz}, 2 \mathrm{H}, \mathrm{H}-10), 4.27-4.33$ (m, 2H, H-5 and H-4), 3.64-3.70 (m, 1H, H-4'), 2.55 (dd, $J=4.8,14.2 \mathrm{~Hz}, 1 \mathrm{H}, \mathrm{H}-6), 2.47$ (dd, $J=7.2,14.2 \mathrm{~Hz}, 1 \mathrm{H}, \mathrm{H}-6$ '), 1.35 (t, $J=7.1 \mathrm{~Hz}, 3 \mathrm{H}, \mathrm{H}-11$ ); $\delta_{\mathrm{C}}$ (100 MHz, $\left.\mathrm{CDCl}_{3}\right) 172.62$ (C-8), 171.03 (C-9), 137.83, 128.82, 128.50, 125.95 (PhC), 87.09 (C-2), 82.23 (C-7), 71.36 (C-4), 63.31 (C-10), 55.94 (C-5), 35.20 (C-6), 14.03 (C-11); m/z (ESI+) 314.25 $\left([\mathrm{M}+\mathrm{Na}]^{+}, 40 \%\right) ;$ HRMS (ESI+) calculated for $\mathrm{C}_{15} \mathrm{H}_{17} \mathrm{NNaO}_{5}\left([\mathrm{M}+\mathrm{Na}]^{+}\right)$314.0999, found 314.1000 .

$(2 R, 5 S, 7 S)$-7-Ethoxycarbonyl-7-hydroxy-8-oxo-2-phenyl-1-aza-3-oxa-bicyclo[3.3.0] octane, (7S)-3a

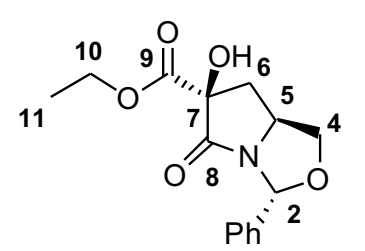

$\mathrm{R}_{f}=0.28\left(1: 1\right.$ EtOAc:PE); colourless oil; $[\alpha]_{\mathrm{D}}^{23}=+98.8(c=0.80$ in $\mathrm{MeCN})$; $v_{\max } / \mathrm{cm}^{-1}\left(\right.$ film) $3423(\mathrm{br}),, 1745(\mathrm{~s}, \mathrm{C}=\mathrm{O}), 1712(\mathrm{~s}, \mathrm{C}=\mathrm{O}) ; \delta_{\mathrm{H}}(400 \mathrm{MHz}$, $\left.\mathrm{CDCl}_{3}\right)$ 7.35-7.44 (m, 5H, PhH), 6.33 (s, 1H, H-2), 4.24-4.35 (m, 4H, H-10, H-5 and H-4), 3.63 (t, $J=8.0 \mathrm{~Hz}, 1 \mathrm{H}, \mathrm{H}-4$ '), 2.94 (dd, $J=6.7,13.3 \mathrm{~Hz}, 1 \mathrm{H}, \mathrm{H}-6), 2.12$ (dd, $J=7.0$, $13.3 \mathrm{~Hz}, 1 \mathrm{H}, \mathrm{H}-6$ '), $1.30(\mathrm{t}, J=7.1 \mathrm{~Hz}, 3 \mathrm{H}, \mathrm{H}-11)$; $\delta_{\mathrm{C}}\left(100 \mathrm{MHz}, \mathrm{CDCl}_{3}\right) 171.25(\mathrm{C}-8), 170.57(\mathrm{C}-$ 9), 137.58, 128.94, 128.55, 126.02 (PhC), 86.99 (C-2), 82.10 (C-7), 72.26 (C-4), 63.01 (C-10), 
55.28 (C-5), 37.45 (C-6), $14.01(\mathrm{C}-11) ; \quad m / z(\mathrm{ESI}+) 314.22\left([\mathrm{M}+\mathrm{Na}]^{+}, 70 \%\right) ;$ HRMS (ESI+) calculated for $\mathrm{C}_{15} \mathrm{H}_{17} \mathrm{NNaO}_{5}\left([\mathrm{M}+\mathrm{Na}]^{+}\right)$314.0999, found 314.1005.

$(2 R, 5 S, 7 R S)$-7-Chloro-7-ethoxycarbonyl-8-oxo-2-phenyl-1-aza-3-oxa-bicyclo[3.3.0] octane, 3b

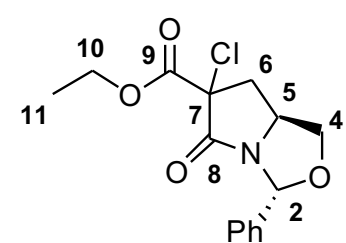

$\mathrm{R}_{f}=0.63\left(1: 1\right.$ EtOAc:PE); courless oil; $v_{\max } / \mathrm{cm}^{-1}$ (film) $1758(\mathrm{~s}, \mathrm{C}=\mathrm{O})$, $1722(\mathrm{~s}, \mathrm{C}=\mathrm{O}) ; \delta_{\mathrm{H}}\left(400 \mathrm{MHz}, \mathrm{CDCl}_{3}\right)$ 7.33-7.47 (m, 5H, PhH), $6.38(\mathrm{~s}, 0.5 \mathrm{H}$, H-2a), 6.32 (s, 0.5H, H-2b), 4.27-4.40 (m, 4H, H-4a, H-4b, H-5b, H-10a, H10b), 4.13-4.20 (m, 0.5H, H-5a), 3.66-3.76 (m, 1H, H-4'a, H-4'b), 3.37 (dd, J=6.5, 13.4 Hz, 0.5H, H-6a), $2.93(\mathrm{dd}, J=6.7,14.1 \mathrm{~Hz}, 0.5 \mathrm{H}, \mathrm{H}-6 \mathrm{~b}), 2.75$ (dd, $J=6.2,14.1 \mathrm{~Hz}, 0.5 \mathrm{H}, \mathrm{H}-6$ 'b), 2.37 (dd, $J$ $=7.0,13.4 \mathrm{~Hz}, 0.5 \mathrm{H}, \mathrm{H}-6$ 'a), 1.37 (t, $J=7.1 \mathrm{~Hz}, 1.5 \mathrm{H}, \mathrm{H}-11 \mathrm{~b}), 1.31$ (t, $J=7.1 \mathrm{~Hz}, 1.5 \mathrm{H}, \mathrm{H}-11 \mathrm{a}) ; \delta_{\mathrm{C}}$ (100 MHz, $\left.\mathrm{CDCl}_{3}\right) 169.09$ (C-8b), 167.86 (C-8a), 166.48 (C-9a), 166.14 (C-9b), 137.25, 137.23, 129.00, 128.98, 128.61, 128.56, 125.98 (PhC), 87.74 (C-2a), 87.10 (C-2b), 71.68 (C-4a), 71.00 (C4b), 70.76 (C-7b), 70.43 (C-7a), 63.70, 63.63 (C-10), 56.12 (C-5b), 55.51 (C-5a), 41.17 (C-6a), 40.75 (C-6b), 14.00, $13.88(\mathrm{C}-11) ; \mathrm{m} / z(\mathrm{ESI}+) 332.19\left([\mathrm{M}+\mathrm{Na}]^{+}, 90 \%\right)$; HRMS (ESI+) calculated for $\mathrm{C}_{15} \mathrm{H}_{16} \mathrm{ClNNaO}_{4}\left([\mathrm{M}+\mathrm{Na}]^{+}\right)$332.0660, found 332.0677 .

\section{3-Phenyl-2-p-tolylsulfonyloxaziridine, 5}

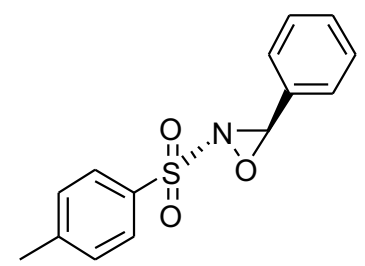

(E)- $N$-Benzylidene-4-methylbenzensulfonamide ( $1 \mathrm{~g}, 3.856 \mathrm{mmol}, 1.0$ eq.) in $\mathrm{CH}_{2} \mathrm{Cl}_{2}(10 \mathrm{~mL})$ was added to the white suspension of $77 \% \mathrm{~m}$-CPBA (950.6 mg, $4.242 \mathrm{mmol}, 1.1$ eq.) and powdered $\mathrm{KOH}$ (757.3 mg, 13.50 mmol, 13.5 eq.) in $10 \mathrm{~mL} \mathrm{CH}_{2} \mathrm{Cl}_{2}$. (prepared at $0^{\circ} \mathrm{C}$ on adding $m$-CPBA to $\mathrm{KOH}$ and warmed to r.t. prior use). After $15 \mathrm{~min}$, the reaction crude is then filtered over Celite and the solvent evaporated to give the pure oxaziridine $(824 \mathrm{mg}, 78 \%) \cdot \mathrm{R}_{f}=0.40(20 \% \mathrm{EtOAc} / \mathrm{PE})$; white solid, $\mathrm{mp}=89-91{ }^{\circ} \mathrm{C}$ [lit. $\left.{ }^{64} 87-89^{\circ} \mathrm{C}\right] ; v_{\max } / \mathrm{cm}^{-1}$ (film) $3068,2930,1350,1168 ; \delta_{\mathrm{H}}\left(400 \mathrm{MHz}, \mathrm{CDCl}_{3}\right) 7.93(\mathrm{~d}, J=8.3$ $\mathrm{Hz}, 2 \mathrm{H}, \mathrm{ArH}), 7.40-7.47(\mathrm{~m}, 7 \mathrm{H}, \mathrm{ArH}), 5.45(\mathrm{~s}, 1 \mathrm{H}), 2.50(\mathrm{~s}, 3 \mathrm{H}) ; \delta_{\mathrm{C}}\left(100 \mathrm{MHz}, \mathrm{CDCl}_{3}\right) 146.36$, 
$131.60,131.35,130.60,130.03,129.42,128.71,128.22,76.33,21.81 ; \mathrm{m} / z$ (ESI-) 274.26 ([M-H]', $65 \%)$.

$(2 R, 5 S, 7 S)$ and $(2 R, 5 S, 7 R)-1-A z a-7-h y d r o x y-7-(N, O$-dimethylcarbamoyl)-3-oxa-8-oxo-2phenyl-bicyclo[3.3.0]octane, 6

To a $1 \mathrm{M}$ THF solution of KHMDS (1.36 mL, $1.357 \mathrm{mmol}, 1.3$ eq.) at $-78^{\circ} \mathrm{C}$ was added 73 (303 $\mathrm{mg}, 1.044 \mathrm{mmol}, 1.0$ eq.) in THF (4 mL). The solution was stirred at $-78^{\circ} \mathrm{C}$ for $40 \mathrm{~min}$, after which oxaziridine 5 (287.4 mg, $1.044 \mathrm{mmol}, 1.0$ eq.) in THF ( $3 \mathrm{~mL})$ was added. After stirring at $-78^{\circ} \mathrm{C}$ for $35 \mathrm{~min}$, the reaction was quenched with sat. aq. $\mathrm{NH}_{4} \mathrm{Cl}$, extracted with EtOAc. The combined organic layers dried with $\mathrm{MgSO}_{4}$, filtered and evaporated to give the crude, which was purified on silica gel (Eluent $0 \%$ to $10 \%$ EtOAc/ $/ \mathrm{CH}_{2} \mathrm{Cl}_{2}$ ) to give the desired product $(182 \mathrm{mg}, 57 \%$, d.r. $=$ $1: 0.2)$

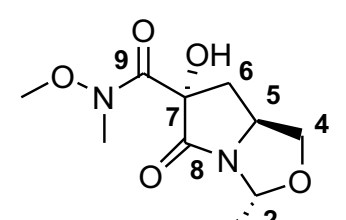

$\mathrm{R}_{f}=0.54\left(1: 1 \mathrm{EtOAc}: \mathrm{CH}_{2} \mathrm{Cl}_{2}\right)$; colourless solid; $v_{\max } / \mathrm{cm}^{-1}$ (film) 3404 (br, -

$\mathrm{OH}), 1710(\mathrm{~s}, \mathrm{C}=\mathrm{O}), 1655(\mathrm{~s}, \mathrm{C}=\mathrm{O}) ; \delta_{\mathrm{H}}\left(400 \mathrm{MHz}, \mathrm{MeCN}_{-} \mathrm{d}_{3}\right)$ 7.36-7.49 (m, $\left.\mathrm{Ph}^{\circ} 5 \mathrm{H}, \mathrm{PhH}\right), 6.20,6.19(\mathrm{~s}, 1 \mathrm{H}, \mathrm{H}-2), 4.84,4.82(\mathrm{~s}, 1 \mathrm{H},-\mathrm{OH}), 4.33-4.40(\mathrm{~m}$, 0.75H, H-5), 4.28-4.32 (m, 1H, H-4), 4.06-4.13 (m, 0.25H, H-5), 3.68, 3.44 (s, 3H, -NMe), 3.54 (t, $J=8.2 \mathrm{~Hz}, 1 \mathrm{H}, \mathrm{H}-4), 3.28,3.23$ (s, 3H, $-\mathrm{OMe}), 2.86$ (dd, $J=7.9,14.3 \mathrm{~Hz}, 0.6 \mathrm{H}, \mathrm{H}-6 \mathrm{~b}), 2.50$ (dd, $J$ $=5.5,14.1 \mathrm{~Hz}, 0.4 \mathrm{H}, \mathrm{H}-6 \mathrm{a}), 2.22$ (dd, $\left.J=7.6,14.0 \mathrm{~Hz}, 0.4 \mathrm{H}, \mathrm{H}-6 \mathrm{a}^{\prime}\right), 2.00$ (dd, $J=3.8,14.3 \mathrm{~Hz}$, 0.6H, H-6b'); $\delta_{\mathrm{C}}\left(100 \mathrm{MHz}, \mathrm{MeCN}-\mathrm{d}_{3}\right)$ 175.12, 173.31 (C-8), 171.28, 171.00 (C-9), 140.26, 139.87, $129.84,129.75,129.70,129.66,129.59,129.51,129.49,129.48,127.17,127.09(\mathrm{PhC}), 89.57$, 87.84 (C-2), 83.19, 81.94 (C-7), $73.01,72.74$ (C-4), 61.58, 61.08 (-NMe), 57.36, 56.78 (C-5), 37.32, $36.20(\mathrm{C}-6), 33.97,33.61(-\mathrm{OMe}) ; \mathrm{m} / \mathrm{z}(\mathrm{ESI}+) 329.24\left([\mathrm{M}+\mathrm{Na}]^{+}, 95 \%\right)$; HRMS (ESI+) calculated for $\mathrm{C}_{15} \mathrm{H}_{18} \mathrm{~N}_{2} \mathrm{NaO}_{5}\left([\mathrm{M}+\mathrm{Na}]^{+}\right)$329.1108, found 329.1121 .

\section{General procedure for Grignard reaction of hydroxy Weinreb amide 6}


The Grignard reagent in THF (2.2 eq. based on amount of 6 used) was added to 6 (1.0 eq.) in THF at $0^{\circ} \mathrm{C}$. The reaction was stirred at $0^{\circ} \mathrm{C}$ to r.t. over a period of $3-4 \mathrm{~h}$, after which it was quenched with sat. aq. $\mathrm{NH}_{4} \mathrm{Cl}$ and extracted with EtOAc. The combined organic layers were dried with anhydrous $\mathrm{MgSO}_{4}$, filtered and the solvents removed in vacuo to give the crude product. Purification via silica gel flash column chromatography (Eluent: EtOAc/PE) gave the respective product as two separate diastereomers about C-7.

$(2 R, \quad 5 S, \quad 7 S)-1-A z a-7-h y d r o x y-7-(4$ 'methylphenylcarbonyl)-3-oxa-8-oxo-2-phenylbicyclo[3.3.0]octane, $(7 S)-7$ a

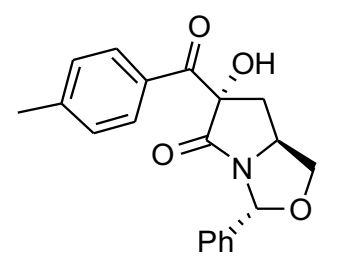

Scale of reaction: $87 \mathrm{mg}(0.284 \mathrm{mmol})$, product $45 \mathrm{mg}, 47 \%$; $\mathrm{R}_{f}=0.51(1: 1$ EtOAc:PE $)$; colourless crystals, $\mathrm{mp}=145-146^{\circ} \mathrm{C} ;[\alpha]_{\mathrm{D}}^{23}=+143.0(c=0.93$ in

$\left.\mathrm{CH}_{2} \mathrm{Cl}_{2}\right) ; v_{\max } / \mathrm{cm}^{-1}$ (film) $3405(\mathrm{br},-\mathrm{OH}), 1709(\mathrm{~s}, \mathrm{C}=\mathrm{O}), 1673(\mathrm{~s}, \mathrm{C}=\mathrm{O}) ; \delta_{\mathrm{H}}$ (400 MHz, MeCN-d $)_{3} 7.85$ (d, J=8.3 Hz, 2H, ArH), 7.48-7.53 (m, 2H, PhH), 7.38-7.47 (m, 3H, PhH), 7.36 (d, J=8.0 Hz, 2H, ArH), 6.30 (s, 1H, H-2), 5.03 (s, 1H, -OH), 4.48 (ddt, J = 6.1, 6.9, $8.5 \mathrm{~Hz}, 1 \mathrm{H}, \mathrm{H}-5), 4.33$ (dd, $J=6.2,8.4 \mathrm{~Hz}, 1 \mathrm{H}, \mathrm{H}-4$ exo), 3.69 (t, $J=8.5 \mathrm{~Hz}, 1 \mathrm{H}, \mathrm{H}-4$ ' endo), 2.68 (dd, $J=6.1,14.4 \mathrm{~Hz}, 1 \mathrm{H}, \mathrm{H}-6$ endo), 2.42 (s, 3H, Ar-Me), 2.40 (dd, J = 7.0, $14.4 \mathrm{~Hz}, 1 \mathrm{H}, \mathrm{H}-6$ 'exo); $\delta_{\mathrm{C}}\left(100 \mathrm{MHz}, \mathrm{MeCN}_{-} \mathrm{d}_{3}\right) 198.95$ (C-8), 174.27 (C-9), 145.88, 139.54, 131.77, 130.78, 130.23, 129.98, 129.63, 127.23 (ArC), 88.38 (C-7), 88.12 (C-2), 72.72 (C-4), 58.02 (C-5), 39.08 (C-6), $21.77(\mathrm{Ar}-\underline{\mathrm{Me}}) ; \mathrm{m} / \mathrm{z}(\mathrm{ESI}+) 360.27\left([\mathrm{M}+\mathrm{Na}]^{+}, 65 \%\right)$; HRMS (ESI+) calculated for $\mathrm{C}_{20} \mathrm{H}_{19} \mathrm{NNaO}_{4}$ $\left([\mathrm{M}+\mathrm{Na}]^{+}\right) 360.1206$, found 360.1222 .

$(2 R, \quad 5 S, \quad 7 R)-1-A z a-7-h y d r o x y-7-(4$ 'methylphenylcarbonyl)-3-oxa-8-oxo-2-phenylbicyclo[3.3.0]octane, $(7 R)-7$ a

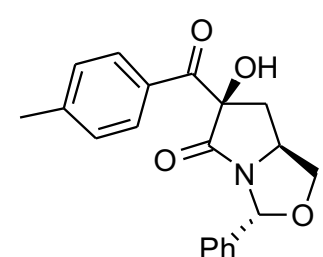

$\mathrm{Ph}^{\prime \prime}$

Product: $9 \mathrm{mg}, 9 \% ; \mathrm{R}_{f}=0.38\left(1: 1\right.$ EtOAc:PE); colourless oil; $[\alpha]_{\mathrm{D}}^{23}=+152.3$ $\left(c=1.03\right.$ in $\left.\mathrm{CH}_{2} \mathrm{Cl}_{2}\right) ; v_{\max } / \mathrm{cm}^{-1}$ (film) 3345 (br, $\left.-\mathrm{OH}\right), 1702(\mathrm{~s}, \mathrm{C}=\mathrm{O}), 1672$ (s, $\mathrm{C}=\mathrm{O}) ; \delta_{\mathrm{H}}\left(400 \mathrm{MHz}, \mathrm{MeCN}-\mathrm{d}_{3}\right) 8.10(\mathrm{~d}, J=8.3 \mathrm{~Hz}, 2 \mathrm{H}, \mathrm{ArH}), 7.35-7.42(\mathrm{~m}$, 
5H, PhH), 7.29 (d, J=8.1 Hz, 2H, ArH), 6.20 (s, 1H, H-2), 4.72 (s, 1H, -OH), 4.32 (dd, J= 6.1, 8.2 Hz, 1H, H-4), 4.14 (apparent dq, $J=6.5,8.3 \mathrm{~Hz}, 1 \mathrm{H}, \mathrm{H}-5$ ), 3.63 (t, $J=8.3 \mathrm{~Hz}, 1 \mathrm{H}, \mathrm{H}-4$ '), 3.13 (dd, $J=6.5,13.1 \mathrm{~Hz}, 1 \mathrm{H}, \mathrm{H}-6), 2.15$ (s, 3H, Ar-Me), 2.12 (dd, $J=7.1,13.2 \mathrm{~Hz}, 1 \mathrm{H}, \mathrm{H}-6$ ' partially obscured by Ar-Me); $\delta_{\mathrm{C}}\left(100 \mathrm{MHz}, \mathrm{MeCN}^{-d_{3}}\right) 197.86$ (C-9), 173.89 (C-8), 145.59, 139.56, 132.38, 131.24, 129.93, 129.92, 129.56, 127.23, 127.01 (ArC), 89.29 (C-7), 88.12 (C-2), 73.43 (C-4), 56.34 (C-5), 40.05 (C-6), $21.74($ Ar-Me $) ; m / z(E S I+) 360.26\left([\mathrm{M}+\mathrm{Na}]^{+}, 95 \%\right)$; HRMS (ESI+) calculated for $\mathrm{C}_{20} \mathrm{H}_{19} \mathrm{NNaO}_{4}\left([\mathrm{M}+\mathrm{Na}]^{+}\right) 360.1206$, found 360.1218 .

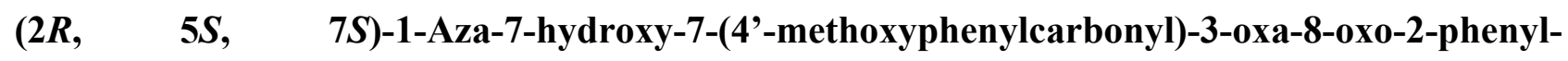
bicyclo[3.3.0]octane, $(7 S)-7 \mathrm{~b}$

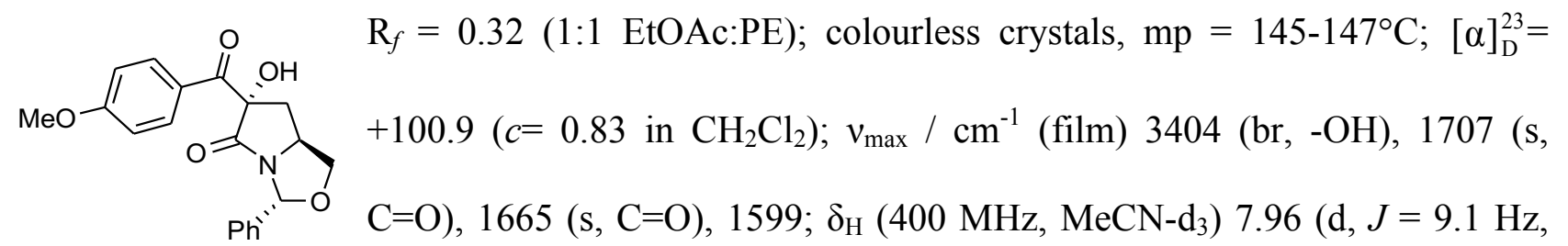
2H, ArH), 7.48-7.54 (m, 2H, PhH), 7.36-7.47 (m, 3H, PhH), 7.05 (d, J=9.1 Hz, 2H, ArH), 6.29 (s, 1H, H-2), 5.04 (s, 1H, -OH), 4.48 (ddt, $J=6.1,7.0,8.5 \mathrm{~Hz}, 1 \mathrm{H}, \mathrm{H}-5), 4.34$ (dd, $J=6.3,8.3 \mathrm{~Hz}, 1 \mathrm{H}$, H-4 exo), 3.88 (s, 3H, -OMe), 3.71 (t, $J=8.5 \mathrm{~Hz}, 1 \mathrm{H}, \mathrm{H}-4$ ' endo), 2.69 (dd, $J=6.1,14.4 \mathrm{~Hz}, 1 \mathrm{H}$, H-6 endo), 2.40 (dd, $J=7.0,14.4 \mathrm{~Hz}, 1 \mathrm{H}, \mathrm{H}-6$ 'exo); $\delta_{\mathrm{C}}\left(100 \mathrm{Mhz}, \mathrm{MeCN}-\mathrm{d}_{3}\right) 197.41$ (C-9), 174.36 (C-8), 165.04, 139.57, 133.28, 129.99, 129.65, 127.25, 126.80, 114.90 (ArC), 88.17 (C-7), 88.13 (C-2), 72.76 (C-4), $58.01(\mathrm{C}-5), 56.47(-\mathrm{OMe}), 39.30(\mathrm{C}-6) ; m / z(\mathrm{ESI}+) 376.27\left([\mathrm{M}+\mathrm{Na}]^{+}, 55 \%\right)$; HRMS (ESI+) calculated for $\mathrm{C}_{20} \mathrm{H}_{19} \mathrm{NNaO}_{5}\left([\mathrm{M}+\mathrm{Na}]^{+}\right) 376.1155$, found 376.1151 .

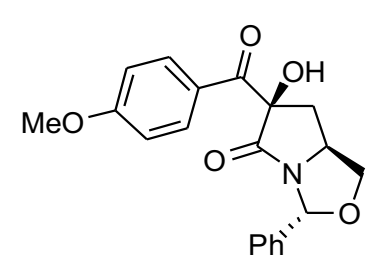

$(2 R, 5 S, 7 R)-1-A z a-7-h y d r o x y-7-(4 '-m e t h o x y p h e n y l c a r b o n y l)-3-0 x a-8-$ oxo-2-phenyl-bicyclo[3.3.0]octane, $(7 R)-7 b$

$\mathrm{R}_{f}=0.24\left(1: 1\right.$ EtOAc:PE); colourless crystals, $\mathrm{mp}=140-142^{\circ} \mathrm{C} ;[\alpha]_{\mathrm{D}}^{23}=$ +94.0 (c=0.51 in $\left.\mathrm{CH}_{2} \mathrm{Cl}_{2}\right) ; v_{\max } / \mathrm{cm}^{-1}$ (film) 3404 (br, -OH), 1707 (s, C=O), 1665 (s, C=O), 1599; $\delta_{\mathrm{H}}\left(400 \mathrm{MHz}, \mathrm{MeCN}_{-} \mathrm{d}_{3}\right) 8.23(\mathrm{~d}, J=9.1 \mathrm{~Hz}, 2 \mathrm{H}, \mathrm{ArH}), 7.29-7.46(\mathrm{~m}, 5 \mathrm{H}, \mathrm{PhH}), 6.98(\mathrm{~d}, J=9.1$ 
$\mathrm{Hz}, 2 \mathrm{H}, \mathrm{ArH}), 6.20$ (s, 1H, H-2), $4.71(\mathrm{~s}, 1 \mathrm{H},-\mathrm{OH}), 4.32$ (dd, $J=6.0,8.2 \mathrm{~Hz}, 1 \mathrm{H}, \mathrm{H}-4$ exo), 4.14 (ddt, $J=6.2,7.0,8.4 \mathrm{~Hz}, 1 \mathrm{H}, \mathrm{H}-5), 3.86$ (s, 3H, -OMe), 3.62 (t, $J=8.5 \mathrm{~Hz}, 1 \mathrm{H}, \mathrm{H}-4$ ' endo), 3.14 (dd, $J=6.5,13.0 \mathrm{~Hz}, 1 \mathrm{H}, \mathrm{H}-6$ exo), 2.11 (dd, $J=7.1,13.0 \mathrm{~Hz}, 1 \mathrm{H}, \mathrm{H}-6$ ' endo); $\delta_{\mathrm{C}}(100 \mathrm{MHz}$, MeCN-d $\left.{ }_{3}\right) 196.26$ (C-9), 174.04 (C-8), 164.90, 139.58, 133.76, 129.91, 129.56, 127.54, 127.23, 114.53 (ArC), 89.26 (C-7), 88.12 (C-2), 73.46 (C-4), 56.37 (C-5), 56.35 (-OMe), 40.15 (C-6); m/z (ESI+) $376.26\left([\mathrm{M}+\mathrm{Na}]^{+}, 80 \%\right)$; HRMS $(\mathrm{ESI}+)$ calculated for $\mathrm{C}_{20} \mathrm{H}_{19} \mathrm{NNaO}_{5}\left([\mathrm{M}+\mathrm{Na}]^{+}\right) 376.1155$, found 376.1165 .

$(2 R, \quad 5 S, \quad 7 S)-1-A z a-7-h y d r o x y-7-(o c t y l c a r b o n y l)-3-o x a-8-0 x 0-2-p h e n y l-b i c y c l o[3.3 .0] o c t a n e$, (7S)-7c

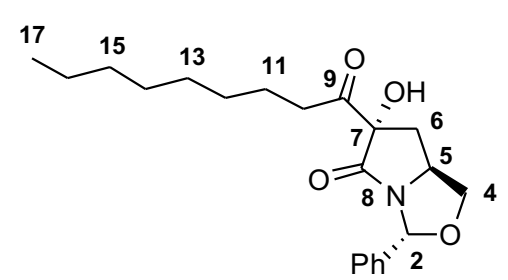

Scale of reaction: $90 \mathrm{mg}(0.294 \mathrm{mmol})$, product: $25 \mathrm{mg}, 24 \%$; $\mathrm{R}_{f}=$ 0.53 (1:1 EtOAc:PE); colourless oil; $[\alpha]_{\mathrm{D}}^{23}=+106.6(c=0.91$ in $\mathrm{CH}_{2} \mathrm{Cl}_{2}$ ); $v_{\max } / \mathrm{cm}^{-1}$ (film) 3403 (br, -OH), 1719 (s, C=O), 1703 (s, $\mathrm{C}=\mathrm{O}) ; \delta_{\mathrm{H}}\left(400 \mathrm{MHz}, \mathrm{MeCN}_{-} \mathrm{d}_{3}\right)$ 7.38-7.47 (m, 5H, PhH), $6.12(\mathrm{~s}, 1 \mathrm{H}, \mathrm{H}-2), 4.67$ (s, 1H, -OH), 4.304.39 (m, 2H, H-5, H-4), 3.63 (t, $J=8.1 \mathrm{~Hz}, 1 \mathrm{H}, \mathrm{H}-4$ '), 2.69 (dt, $J=7.2,18.1 \mathrm{~Hz}, 1 \mathrm{H}, \mathrm{H}-10), 2.67$ (dt, $J=7.2,18.1 \mathrm{~Hz}, 1 \mathrm{H}, \mathrm{H}-10$ '), $2.51(\mathrm{dd}, J=5.4,14.3 \mathrm{~Hz}, 1 \mathrm{H}, \mathrm{H}-6), 2.24$ (dd, $J=6.8,14.2 \mathrm{~Hz}$, 1H, H-6’ ), 1.54-1.59 (m, 2H, H-11), 1.26-1.35 (m, 10H, H-12, H-13, H-14, H-15, H-16), 0.89 (t, J $=7.0 \mathrm{~Hz}, 3 \mathrm{H}, \mathrm{H}-17) ; \delta_{\mathrm{C}}\left(100 \mathrm{MHz}, \mathrm{MeCN}_{-} \mathrm{d}_{3}\right) 210.13$ (C-9), 174.17 (C-8), 139.74, 129.90, 129.57, 127.19 (PhC), 88.65 (C-7), 87.82 (C-2), 72.71 (C-4), 57.67 (C-5), 38.47 (C-10), 36.75 (C-6), 32.61, 30.11, 29.61, 29.77, 24.10 (C-11), 23.39 (C-12, C-13, C-14, C-15, C-16), 14.38 (C-17); m/z (ESI+) $382.38\left([\mathrm{M}+\mathrm{Na}]^{+}, 70 \%\right)$; HRMS $(\mathrm{ESI}+)$ calculated for $\mathrm{C}_{21} \mathrm{H}_{29} \mathrm{NNaO}_{4}\left([\mathrm{M}+\mathrm{Na}]^{+}\right) 382.1989$, found 382.1996.

$(2 R, 5 S, 7 R)$-1-Aza-7-hydroxy-7-(octylcarbonyl)-3-oxa-8-oxo-2-phenyl-bicyclo[3.3.0]octane, (7R)-7c 


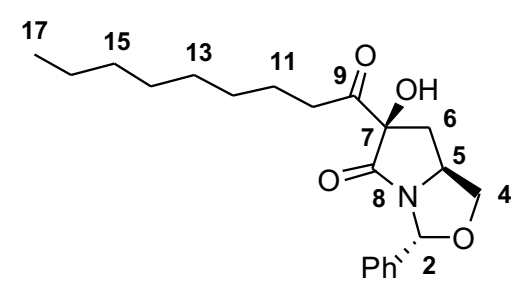

Product: $7 \mathrm{mg}, 7 \% ; \mathrm{R}_{f}=0.38\left(1: 1\right.$ EtOAc:PE); colourless oil; $[\alpha]_{\mathrm{D}}^{23}=$ $+190.1\left(c=0.73\right.$ in $\left.\mathrm{CH}_{2} \mathrm{Cl}_{2}\right) ; v_{\max } / \mathrm{cm}^{-1}$ (film) $3373(\mathrm{br},-\mathrm{OH}), 1694$

(s, br with shoulder towards larger wavenumber, $\mathrm{C}=\mathrm{O}) ; \delta_{\mathrm{H}}(400$ MHz, MeCN-d $\mathrm{d}_{3}$ ) 7.37-7.43 (m, 5H, PhH), 6.16 (s, 1H, H-2), 4.48 (s, 1H, -OH), 4.32 (dd, $J=6.0$, $8.2 \mathrm{~Hz}, 1 \mathrm{H}, \mathrm{H}-4), 4.13-4.20$ (m, 1H, H-5), 3.57 (t, $J=8.4 \mathrm{~Hz}, 1 \mathrm{H}, \mathrm{H}-4$ '), 2.85 (dd, $J=6.8,13.4 \mathrm{~Hz}$, 1H, H-6), 2.67 (t, $J=7.2 \mathrm{~Hz}, 2 \mathrm{H}, \mathrm{H}-10$ ), 1.97 (dd, 1H, H-6', partially obscured by MeCN-d signal), 1.48-1.55 (m, 2H, H-11), 1.19-1.34 (m, 10H, H-12, H-13, H-14, H-15, H-16), 0.87 (t, $J=$ $7.0 \mathrm{~Hz}, 3 \mathrm{H}, \mathrm{H}-17) ; \delta_{\mathrm{C}}\left(100 \mathrm{MHz}, \mathrm{MeCN}-\mathrm{d}_{3}\right) 209.29$ (C-9), 173.26 (C-8), 139.62, 129.93, 129.57, 127.19 (PhC), 88.96 (C-7), 88.01 (C-2), 73.61 (C-4), 56.40 (C-5), 37.91 (C-6), 37.33 (C-10), 32.55, 30.02, 29.88, 29.71 (C-13, C-14, C-15, C-16),24.02 (C-11), 23.36 (C-12), 14.38 (C-17); m/z (ESI+) $382.39\left([\mathrm{M}+\mathrm{Na}]^{+}, 70 \%\right)$; HRMS (ESI+) calculated for $\mathrm{C}_{21} \mathrm{H}_{29} \mathrm{NNaO}_{4}\left([\mathrm{M}+\mathrm{Na}]^{+}\right) 382.1989$, found 382.1989 .

$(2 R, 5 S, 7 S)-1-A z a-7-(b u t y l c a r b o n y l)-7-h y d r o x y-3-o x a-8-o x o-2-p h e n y l-b i c y c l o[3.3 .0]$ octane, (7S)-7d

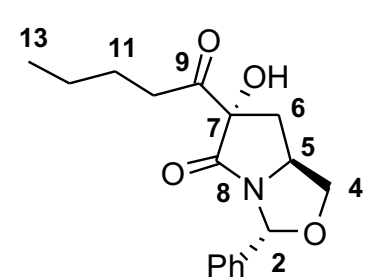

Scale of reaction: $108 \mathrm{mg}(0.353 \mathrm{mmol})$, product: $37 \mathrm{mg}, 35 \% \mathrm{R}_{f}=0.53(1: 1$ EtOAc:PE); colourless oil; $[\alpha]_{\mathrm{D}}^{23}=+137.0\left(c=1.06\right.$ in $\left.\mathrm{CH}_{2} \mathrm{Cl}_{2}\right) ; v_{\max } / \mathrm{cm}^{-1}$ (film) 3405 (br, -OH), 1719 (s, C=O), 1701 (s, C=O); $\delta_{\mathrm{H}}(400 \mathrm{MHz}, \mathrm{MeCN}$ d $\mathrm{d}_{3}$ 7.34-7.49 (m, 5H, PhH), 6.12 (s, 1H, H-2), 4.68 (s, 1H, -OH), 4.35-4.41 (m, 1H, H-5), 4.32 (dd, $J=6.1,7.6 \mathrm{~Hz}, 1 \mathrm{H}, \mathrm{H}-4), 3.63$ (t, $J=8.1 \mathrm{~Hz}, 1 \mathrm{H}, \mathrm{H}-4$ '), 2.71 (dt, $J=7.2,18.1 \mathrm{~Hz}, 1 \mathrm{H}, \mathrm{H}-10), 2.69$ (dt, $\left.J=7.2,18.1 \mathrm{~Hz}, 1 \mathrm{H}, \mathrm{H}-10^{\prime}\right), 2.51$ (dd, $\left.J=5.5,14.3 \mathrm{~Hz}, 1 \mathrm{H}, \mathrm{H}-6\right), 2.24$ (dd, $J=6.9,14.3 \mathrm{~Hz}$, 1H, H-6'), 1.52-1.60 (m, 2H, H-11), 1.28-1.39 (m, 2H, H-12), 0.92 (t, $J=7.3 \mathrm{~Hz}, 3 \mathrm{H}, \mathrm{H}-13) ; \delta_{\mathrm{C}}$ (100 MHz, MeCN-d 3 ) 210.15 (C-9), 174.18 (C-8), 139.75, 129.91, 129.58, 127.20 (PhC), 88.67 (C7), 87.82 (C-2), 72.71 (C-4), 57.67 (C-5), 38.20 (C-10), 36.76 (C-6), 26.11 (C-11), 22.88 (C-12), $14.16(\mathrm{C}-13) ; \mathrm{m} / \mathrm{z}(\mathrm{ESI}+) 326.30\left([\mathrm{M}+\mathrm{Na}]^{+}, 50 \%\right)$; HRMS (ESI+) calculated for $\mathrm{C}_{17} \mathrm{H}_{21} \mathrm{NNaO}_{4}$ $\left([\mathrm{M}+\mathrm{Na}]^{+}\right)$326.1363, found 326.1371 . 


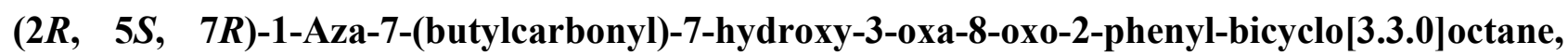
$(7 R)-7 d$

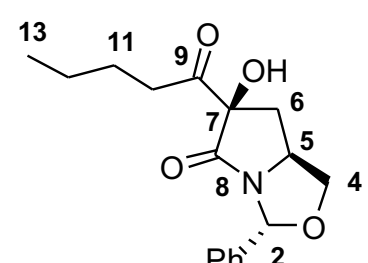

Product: $3 \mathrm{mg}, 3 \% ; \mathrm{R}_{f}=0.39$ (1:1 EtOAc:PE); colourless oil; $[\alpha]_{\mathrm{D}}^{23}=+156.2$ ( $c=0.66$ in $\left.\mathrm{CH}_{2} \mathrm{Cl}_{2}\right) ; v_{\max } / \mathrm{cm}^{-1}$ (film) $3383(\mathrm{br},-\mathrm{OH}), 1696$ (s, br shoulder towards larger wavenumber, $\mathrm{C}=\mathrm{O}) ; \delta_{\mathrm{H}}\left(400 \mathrm{MHz}, \mathrm{MeCN}-\mathrm{d}_{3}\right)$ 7.34-7.44 (m, 5H, PhH), 6.16 (s, 1H, H-2), 4.49 (s, 1H, -OH), 4.32 (dd, J=6.0, 8.2 Hz, 1H, H-4), 4.16 (apparent dq, $J=6.8,8.5 \mathrm{~Hz}, 1 \mathrm{H}, \mathrm{H}-5), 3.57$ (t, $J=8.4 \mathrm{~Hz}, 1 \mathrm{H}, \mathrm{H}-4$ '), 2.85 (dd, $J=6.8,13.4 \mathrm{~Hz}, 1 \mathrm{H}, \mathrm{H}-6$ ), $2.68(\mathrm{t}, J=7.2 \mathrm{~Hz}, 2 \mathrm{H}, \mathrm{H}-10), 1.95$ (dd, $J=6.9 \mathrm{~Hz}, 1 \mathrm{H}, \mathrm{H}-6$ ', partially obscured by MeCN-d 3 signal), 1.44-1.55 (m, 2H, H-11), 1.19-1.31 (m, 2H, H-12), 0.85 (t, J = 7.4 Hz, 3H, H-13); $\delta_{\mathrm{C}}(100$ MHz, MeCN-d 3 ) 209.36 (C-9), 173.27 (C-8), 139.61, 130.56, 129.93, 129.58, 127.20 (PhC), 88.96 (C-7), 88.01 (C-2), 73.60 (C-4), 56.40 (C-5), 37.95 (C-6), 37.08 (C-10), 26.13 (C-11), 22.83 (C-12), $14.08(\mathrm{C}-13) ; \mathrm{m} / \mathrm{z}(\mathrm{ESI}+) 326.26\left([\mathrm{M}+\mathrm{Na}]^{+}, 65 \%\right)$; HRMS (ESI+) calculated for $\mathrm{C}_{17} \mathrm{H}_{21} \mathrm{NNaO}_{4}$ $\left([\mathrm{M}+\mathrm{Na}]^{+}\right)$326.1363, found 326.1373 .

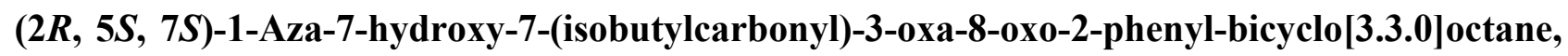
(7S)-7e

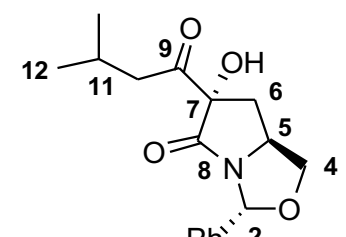

Scale of reaction: $86 \mathrm{mg}(0.281 \mathrm{mmol})$, product: $5 \mathrm{mg}, 6 \% ; \mathrm{R}_{f}=0.57(1: 1$ EtOAc/PE); colourless oil; $[\alpha]_{\mathrm{D}}^{23}=+180.3\left(c=0.57\right.$ in $\left.\mathrm{CH}_{2} \mathrm{Cl}_{2}\right) ; v_{\max } / \mathrm{cm}^{-1}$ (film) 3404 (br, -OH), $1718(\mathrm{~s}, \mathrm{C}=\mathrm{O}), 1700\left(\mathrm{~s}, \mathrm{C}=\mathrm{O} ; \delta_{\mathrm{H}}\left(400 \mathrm{MHz}, \mathrm{MeCN}-\mathrm{d}_{3}\right)\right.$ 7.35-7.49 (m, 5H, PhH), 6.12 (s, 1H, H-2), 4.66 (s, 1H, -OH), 4.29-4.40 (m, 2H, H-5, H-4), 3.63 (t, $J=8.0 \mathrm{~Hz}, 1 \mathrm{H}, \mathrm{H}-4$ '), 2.64 (dd, $J=6.7,17.9 \mathrm{~Hz}, 1 \mathrm{H}, \mathrm{H}-10), 2.56$ (dd, $J=6.7,17.9 \mathrm{~Hz}, 1 \mathrm{H}, \mathrm{H}-10$ '), $2.50(\mathrm{dd}, J=5.5,14.3 \mathrm{~Hz}, 1 \mathrm{H}, \mathrm{H}-6), 2.23(\mathrm{dd}, J=6.9,14.3 \mathrm{~Hz}, 1 \mathrm{H}, \mathrm{H}-6$ '), $2.13-2.18$ (m, 1H, H-11), $0.94(\mathrm{~d}, J=3.5 \mathrm{~Hz}, 3 \mathrm{H}, \mathrm{H}-12), 0.92$ (d, $J=3.5 \mathrm{~Hz}, 3 \mathrm{H}, \mathrm{H}-12$ '); $\delta_{\mathrm{C}}\left(100 \mathrm{MHz}, \mathrm{MeCN}-\mathrm{d}_{3}\right) 209.63$ (C-9), 174.08 (C-8), 139.77, 129.90, 129.58, 127.20 (PhC), 88.65 (C-7), 87.82 (C-2), 72.71 (C-4), 57.63 (C-5), 47.15 (C-10), 36.69 (C-6), 24.73 (C-11), 22.75, 22.68 (C-12, C-12'); m/z (ESI+) 
$326.31\left([\mathrm{M}+\mathrm{Na}]^{+}, 100 \%\right)$; HRMS $(\mathrm{ESI}+)$ calculated for $\mathrm{C}_{17} \mathrm{H}_{21} \mathrm{NNaO}_{4}\left([\mathrm{M}+\mathrm{Na}]^{+}\right) 327.1396$, found 327.1415.

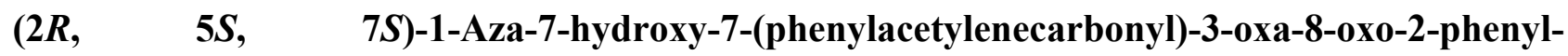
bicyclo[3.3.0]octane, $(7 S)-7 f$

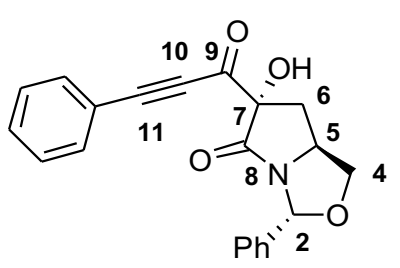

Scale of reaction: $99 \mathrm{mg}(0.323 \mathrm{mmol})$, product: $55 \mathrm{mg}, 49 \%$; $\mathrm{R}_{f}=0.54$ (1:1 EtOAc:PE); white solid, $\mathrm{mp}=154-156^{\circ} \mathrm{C} ;[\alpha]_{\mathrm{D}}^{23}=+151.1(c=1.12$ in

$\left.\mathrm{CH}_{2} \mathrm{Cl}_{2}\right) ; v_{\max } / \mathrm{cm}^{-1}$ (film) 3405 (br, -OH), 2195 (s, alkyne), 1710 (s, $\mathrm{C}=\mathrm{O}), 1675(\mathrm{~s}, \mathrm{C}=\mathrm{O}) ; \delta_{\mathrm{H}}\left(400 \mathrm{MHz}, \mathrm{MeCN}_{-} \mathrm{d}_{3}\right)$ 7.66-7.71 (m, 2H, PhH), 7.57-7.63 (m, 1H, PhH), 7.36-7.56 (m, 7H, PhH), 6.24 (s, 1H, H-2), 4.85 (s, 1H, -OH), 4.46 (ddt, J= 5.6, 6.7, 8.1 Hz, 1H, H5), 4.39 (dd, $J=6.1,7.9 \mathrm{~Hz}, 1 \mathrm{H}, \mathrm{H}-4), 3.73$ (t, $J=8.3 \mathrm{~Hz}, 1 \mathrm{H}, \mathrm{H}-4$ '), 2.83 (dd, $J=5.4,14.7 \mathrm{~Hz}$, 1H, H-6), 2.44 (dd, $J=7.0,14.6$ Hz, 1H, H-6'); $\delta_{\mathrm{C}}\left(100 \mathrm{MHz}, \mathrm{MeCN}-\mathrm{d}_{3}\right) 186.07$ (C-9), 173.34 (C8), 139.51, 134.26, 132.85, 130.09, 129.99, 129.62, 127.19, 119.78 (PhC), 97.87 (C-11), 89.92 (C7), 88.13 (C-2), 85.99 (C-10), 72.96 (C-4), 57.73 (C-5), $36.98(\mathrm{C}-6) ; m / z(\mathrm{ESI}+) 370.29\left([\mathrm{M}+\mathrm{Na}]^{+}\right.$, $100 \%$ ); HRMS (ESI+) calculated for $\mathrm{C}_{21} \mathrm{H}_{18} \mathrm{NO}_{4}\left([\mathrm{M}+\mathrm{H}]^{+}\right)$348.1230, found 348.1224.

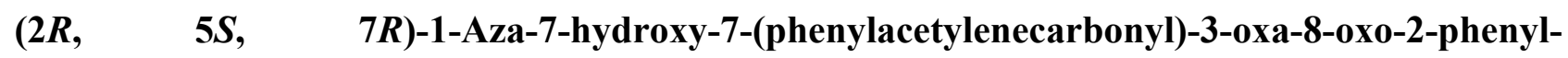
bicyclo[3.3.0]octane, $(7 R)-7 f$

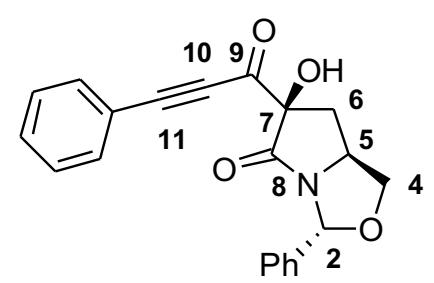

Product: $25 \mathrm{mg}, 22 \% ; \mathrm{R}_{f}=0.39\left(1: 1\right.$ EtOAc:PE); colourless oil; $[\alpha]_{\mathrm{D}}^{23}=$ $+42.9\left(c=1.08\right.$ in $\left.\mathrm{CH}_{2} \mathrm{Cl}_{2}\right) ; v_{\max } / \mathrm{cm}^{-1}$ (film) 3404 (br, $\left.-\mathrm{OH}\right), 2195$ (s, alkyne), $1715(\mathrm{~s}, \mathrm{C}=\mathrm{O}), 1666(\mathrm{~s}, \mathrm{C}=\mathrm{O}) ; \delta_{\mathrm{H}}\left(400 \mathrm{MHz}, \mathrm{MeCN}-\mathrm{d}_{3}\right) 7.52-$ $7.58(\mathrm{~m}, 1 \mathrm{H}, \mathrm{PhH})$, 7.39-7.48 (m, 6H, PhH), 7.28-7.34 (m, 3H, PhH), 6.25 (s, 1H, H-2), 4.74 (s, 1H, -OH), 4.30-4.46 (m, 2H, H-4, H-5), 3.66 (t, J = 7.8 Hz, 1H, H-4'), $3.07(\mathrm{dd}, J=7.0,14.0 \mathrm{~Hz}, 1 \mathrm{H}, \mathrm{H}-6), 2.17\left(\mathrm{dd}, J=6.6,14.0 \mathrm{~Hz}, 1 \mathrm{H}, \mathrm{H}-6\right.$ '); $\delta_{\mathrm{C}}\left(100 \mathrm{MHz}, \mathrm{MeCN}-\mathrm{d}_{3}\right)$ 185.02 (C-9), 171.58 (C-8), 139.60, 134.15, 132.76, 129.99, 129.96, 129.58, 127.10, 119.60 (PhC), 97.05 (C-11), 90.10 (C-7), 87.91 (C-2), 85.08 (C-10), 73.72 (C-4), 56.45 (C-5), 37.17 (C-6); m/z 
(ESI+) $370.31\left([\mathrm{M}+\mathrm{Na}]^{+}, 100 \%\right)$; HRMS (ESI+) calculated for $\mathrm{C}_{21} \mathrm{H}_{18} \mathrm{NO}_{4}\left([\mathrm{M}+\mathrm{H}]^{+}\right) 348.1230$, found 348.1232 .

$(2 R, \quad 5 S$ 7S)-1-Aza-7-hydroxy-7-(butylacetylenecarbonyl)-3-oxa-8-oxo-2-phenylbicyclo[3.3.0]octane, $(7 S)-7 \mathrm{~g}$

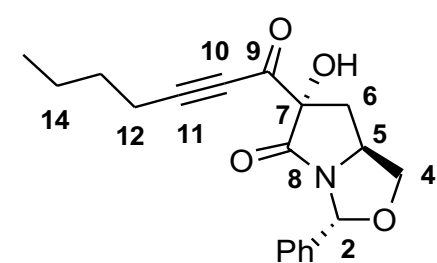

Scale of reaction: $100 \mathrm{mg}(0.326 \mathrm{mmol})$, product: $53 \mathrm{mg}, 50 \% ; \mathrm{R}_{f}=0.51$ $\left(1: 1\right.$ EtOAc:PE); colourless oil; $[\alpha]_{\mathrm{D}}^{23}=+176.9\left(c=1.28\right.$ in $\left.\mathrm{CH}_{2} \mathrm{Cl}_{2}\right) ; v_{\max }$

/ $\mathrm{cm}^{-1}$ (film) 3416 (br, -OH), 2211 (s, alkyne), 1712 (s, C=O), 1680 (s, $\mathrm{C}=\mathrm{O}) ; \delta_{\mathrm{H}}\left(400 \mathrm{MHz}, \mathrm{MeCN}-\mathrm{d}_{3}\right) 7.37-7.49(\mathrm{~m}, 5 \mathrm{H}, \mathrm{PhH}), 6.15(\mathrm{~s}, 1 \mathrm{H}, \mathrm{H}-2), 4.71(\mathrm{~d}, J=0.9 \mathrm{~Hz}, 1 \mathrm{H}$, -OH), 4.34-4.46 (m, 2H, H-5, H-4), 3.65 (t, $J=7.8 \mathrm{~Hz}, 1 \mathrm{H}, \mathrm{H}-4$ '), 2.74 (dd, $J=4.9,14.6 \mathrm{~Hz}, 1 \mathrm{H}$, H-6), 2.51 (t, $J=7.0 \mathrm{~Hz}, 2 \mathrm{H}, \mathrm{H}-12), 2.35$ (dd, $J=7.1,14.6 \mathrm{~Hz}, 1 \mathrm{H}, \mathrm{H}-6$ ') , 1.54-1.65 (m, 2H, H-13), 1.39-1.51 (m, 2H, H-14), 0.95 (t, $J=7.3 \mathrm{~Hz}, 3 \mathrm{H}, \mathrm{H}-15) ; \delta_{\mathrm{C}}\left(100 \mathrm{MHz}, \mathrm{MeCN}_{-} \mathrm{d}_{3}\right) 185.89$ (C-9), 173.23 (C-8), 139.55, 129.98, 129.61, 127.17 (PhC), 103.23 (C-11), 89.90 (C-7), 88.07 (C-2), 78.75 (C-10), 72.94 (C-4), 57.78 (C-5), 36.76 (C-6), 30.22 (C-13), 22.70 (C-14), 19.36 (C-12), 13.75 (C15); $m / z(\mathrm{ESI}+) 350.30\left([\mathrm{M}+\mathrm{Na}]^{+}, 100 \%\right)$; HRMS $(\mathrm{ESI}+)$ calculated for $\mathrm{C}_{19} \mathrm{H}_{21} \mathrm{NNaO}_{4}\left([\mathrm{M}+\mathrm{Na}]^{+}\right)$ 350.1363 , found 350.1356 .

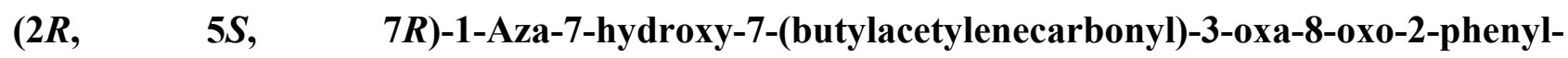
bicyclo[3.3.0]octane, $(7 R)-7 \mathrm{~g}$

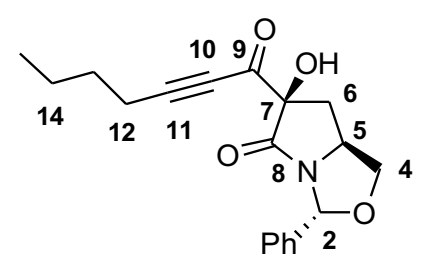

Product: $17 \mathrm{mg}, 16 \% ; \mathrm{R}_{f}=0.37\left(1: 1\right.$ EtOAc:PE); colourless oil; $[\alpha]_{\mathrm{D}}^{23}=$ $+122.0\left(c=0.95\right.$ in $\left.\mathrm{CH}_{2} \mathrm{Cl}_{2}\right) ; v_{\max } / \mathrm{cm}^{-1}$ (film) $3420(\mathrm{br},-\mathrm{OH}), 2210(\mathrm{~s}$, alkyne), $1718(\mathrm{~s}, \mathrm{C}=\mathrm{O}), 1670(\mathrm{~s}, \mathrm{C}=\mathrm{O}) ; \delta_{\mathrm{H}}\left(400 \mathrm{MHz}, \mathrm{MeCN}-\mathrm{d}_{3}\right)$ 7.34$7.48(\mathrm{~m}, 5 \mathrm{H}, \mathrm{PhH}), 6.21$ (s, 1H, H-2), $4.60(\mathrm{~s}, 1 \mathrm{H},-\mathrm{OH}), 4.37$ (dd, J = 6.0, 8.0 Hz, 1H, H-4), 4.22 (apparent dq, $J=6.7,8.7 \mathrm{~Hz}, 1 \mathrm{H}, \mathrm{H}-5), 3.62$ (t, $J=8.4 \mathrm{~Hz}, 1 \mathrm{H}, \mathrm{H}-4$ '), 2.96 (dd, $J=7.0,13.9 \mathrm{~Hz}$, 1H, H-6), 2.38 (t, $J=7.1 \mathrm{~Hz}, 2 \mathrm{H}, \mathrm{H}-12), 2.10$ (dd, $J=6.6,14.0 \mathrm{~Hz}, 1 \mathrm{H}, \mathrm{H}-6$ '), 1.41-1.50 (m, 2H, H-13), 1.26-1.39 (m, 2H, H-14), 0.85 (t, $J=7.3 \mathrm{~Hz}, 3 \mathrm{H}, \mathrm{H}-15)$; $\delta_{\mathrm{C}}\left(100 \mathrm{MHz}, \mathrm{MeCN}-\mathrm{d}_{3}\right) 185.08$ 
(C-9), 171.68 (C-8), 139.64, 129.96, 129.60, 127.11 (PhC), 102.49 (C-11), 89.90 (C-7), 87.96 (C-

2), 77.77 (C-10), 73.61 (C-4), 56.45 (C-5), 37.07 (C-6), 30.17 (C-13), 22.71 (C-14), 19.34 (C-12), $13.69(\mathrm{C}-15) ; \mathrm{m} / z(\mathrm{ESI}+) 350.31\left([\mathrm{M}+\mathrm{Na}]^{+}, 100 \%\right) ; \mathrm{HRMS}(\mathrm{ESI}+)$ calculated for $\mathrm{C}_{19} \mathrm{H}_{21} \mathrm{NNaO}_{4}$ $\left([\mathrm{M}+\mathrm{Na}]^{+}\right) 350.1363$, found 350.1372 .

\section{General procedure for acidic oxazolidine hydrolysis to 2-hydroxypyrrolidinones (2S)-8a-g}

To a stirred solution of (7S)-91/7a-g (1.0 eq.) in acetone (approx. concentration $0.06 \mathrm{M}$, with one small drop of water) at r.t was added Amberlyst 15 (3.0 eq.). After stirring at r.t. for 22-24 h, the reaction mixture was filtered and the resin rinsed with acetone. The solvent was evaporated to give the crude, which was purified via silica gel flash column chromatography (Eluent: EtOAc, then 5\% $\mathrm{MeOH} / \mathrm{EtOAc})$ to give the respective product.

(2S, 4R)-2-Hydroxy-2-(4'-methylphenylcarbonyl)-4-hydroxymethyl-1-oxo-pyrrolidinone, (2S)$8 \mathbf{a}$

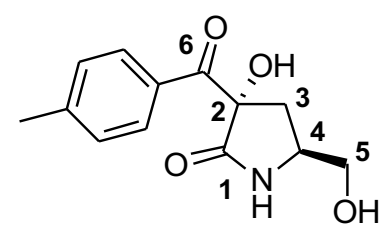

Scale of reaction: $37 \mathrm{mg}(0.110 \mathrm{mmol})$, product: $23 \mathrm{mg}, 83 \%$; $\mathrm{R}_{f}=0.19$ (EtOAc); white solid, $\mathrm{mp}=179-180^{\circ} \mathrm{C} ;[\alpha]_{\mathrm{D}}^{23}=+17.4(c=0.95$ in $\mathrm{MeOH})$; $v_{\max } / \mathrm{cm}^{-1}$ (film) 3496 (br, -NH-), $3276(\mathrm{br},-\mathrm{OH}), 1685$ (s, C=O), $1673(\mathrm{~s}$, $\mathrm{C}=\mathrm{O}) ; \delta_{\mathrm{H}}\left(400 \mathrm{MHz}\right.$, Acetone-d $\left.{ }_{6}\right) 8.02(\mathrm{~d}, J=8.3 \mathrm{~Hz}, 2 \mathrm{H}, \mathrm{ArH}), 7.41$ (br s, 1H, -NH-), $7.30(\mathrm{~d}, J=$ $7.9 \mathrm{~Hz}, 2 \mathrm{H}, \mathrm{ArH}), 5.30(\mathrm{~s}, 1 \mathrm{H},-\mathrm{OH}), 4.13\left(\mathrm{t}, J=5.4 \mathrm{~Hz}, 1 \mathrm{H},-\mathrm{CH}_{2} \underline{\mathrm{OH}}\right), 3.90-4.01(\mathrm{~m}, 1 \mathrm{H}, \mathrm{H}-4)$, $3.72(\mathrm{dd}, J=4.6,10.9 \mathrm{~Hz}, 1 \mathrm{H}, \mathrm{H}-5), 3.58$ (dt, $J=4.6,10.8 \mathrm{~Hz}, 1 \mathrm{H}, \mathrm{H}-5$ ') 2.64 (dd, $J=6.6,13.9$ Hz, 1H, H-3), 2.40 (s, 3H, Ar-Me), 2.23 (dd, $J=7.3,13.9 \mathrm{~Hz}, 1 \mathrm{H}, \mathrm{H}-3$ '); $\delta_{\mathrm{C}}(100 \mathrm{MHz}$, Acetoned $\left.{ }_{6}\right) 199.70$ (C-6), 174.96 (C-1), 144.83, 132.25, 130.88, 129.74 (ArC), 83.54 (C-2), 64.85 (C-5), 54.49 (C-4), 38.03 (C-3), 21.58 (Ar-Me); m/z (ESI+) $272.22\left([\mathrm{M}+\mathrm{Na}]^{+}, 65 \%\right)$; HRMS (ESI+) calculated for $\mathrm{C}_{13} \mathrm{H}_{16} \mathrm{NO}_{4}\left([\mathrm{M}+\mathrm{H}]^{+}\right)$250.1074, found 250.1086 . 
$(2 S)-8 b$

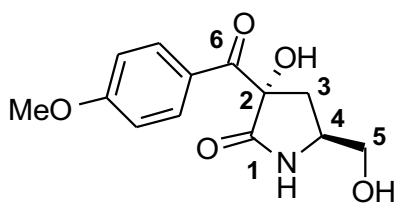

Scale of reaction: $15 \mathrm{mg}(0.0424 \mathrm{mmol})$, product: $10 \mathrm{mg}, 89 \%$; $\mathrm{R}_{f}=0.14$ (EtOAc); white solid, $\mathrm{mp}=153-154^{\circ} \mathrm{C} ;[\alpha]_{\mathrm{D}}^{23}=-23.5(c=0.56$ in $\mathrm{MeCN}) ;$

$v_{\max } / \mathrm{cm}^{-1}$ (film) 3481 (br, -NH-), 3285 (br, -OH), 1687 (s, C=O), 1667

$(\mathrm{s}, \mathrm{C}=\mathrm{O}) ; \delta_{\mathrm{H}}\left(400 \mathrm{MHz}, \mathrm{MeCN}-\mathrm{d}_{3}\right) 8.05(\mathrm{~d}, J=9.1 \mathrm{~Hz}, 2 \mathrm{H}, \operatorname{ArH}), 7.00(\mathrm{~d}, J=9.1 \mathrm{~Hz}, 2 \mathrm{H}, \operatorname{ArH})$, 6.74 (br s, 1H, -NH-), 4.86 (s, 1H, -OH), 3.89-3.97 (m, 1H, H-4), 3.87 (s, 3H, -OMe), 3.66 (ddd, $J=$ 3.9, 5.1, $11.4 \mathrm{~Hz}, 1 \mathrm{H}, \mathrm{H}-5), 3.46$ (dt, $J=6.0,11.6 \mathrm{~Hz}, 1 \mathrm{H}, \mathrm{H}-5$ '), 3.13 (dd, $J=5.1,6.4 \mathrm{~Hz}, 1 \mathrm{H},-$ $\mathrm{CH}_{2} \underline{\mathrm{OH}}$ ), $2.53(\mathrm{dd}, J=7.0,14.1 \mathrm{~Hz}, 1 \mathrm{H}, \mathrm{H}-3), 2.18$ (dd, $J=7.0,14.3 \mathrm{~Hz}, 1 \mathrm{H}, \mathrm{H}-3$ ', partially obscured by water signal); $\delta_{\mathrm{C}}\left(100 \mathrm{MHz}, \mathrm{MeCN}_{-} \mathrm{d}_{3}\right) 198.21$ (C-6), 175.51 (C-1), 165.00, 133.19, 126.74, 114.77 (ArC), 83.18 (C-2), 64.30 (C-5), 56.42 (-OMe), 54.64 (C-4), 38.00 (C-3); m/z $(\mathrm{ESI}+) 288.23\left([\mathrm{M}+\mathrm{Na}]^{+}, 100 \%\right)$; HRMS $(\mathrm{ESI}+)$ calculated for $\mathrm{C}_{13} \mathrm{H}_{15} \mathrm{NNaO} 5\left([\mathrm{M}+\mathrm{Na}]^{+}\right) 288.0842$, found 288.0857 .

(2S, 4R)-2-Hydroxy-4-hydroxymethyl-2-(octylcarbonyl)-1-oxo-pyrrolidinone, $(2 S)-8 \mathrm{c}$

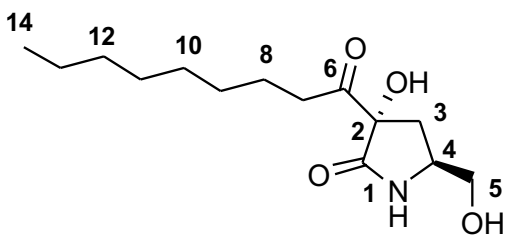

Scale of reaction: $15 \mathrm{mg}(0.417 \mathrm{mmol})$, product: $11 \mathrm{mg}, 97 \%$; $\mathrm{R}_{f}=$ $0.28\left(\right.$ EtOAc); colourless crystals, $\mathrm{mp}=101-103^{\circ} \mathrm{C} ;[\alpha]_{\mathrm{D}}^{23}=+31.0$ $(c=1.22$ in $\mathrm{MeOH}) ; v_{\max } / \mathrm{cm}^{-1}$ (film) 3298 (br), $1716(\mathrm{~s}, \mathrm{C}=\mathrm{O})$,

$1689(\mathrm{~s}, \mathrm{C}=\mathrm{O}) ; \delta_{\mathrm{H}}\left(400 \mathrm{MHz}, \mathrm{MeCN}-\mathrm{d}_{3}\right) 6.61($ br s, $1 \mathrm{H},-\mathrm{NH}-), 4.42(\mathrm{~s}, 1 \mathrm{H},-\mathrm{OH}), 3.77-3.83(\mathrm{~m}$, 1H, H-4), 3.61 (dt, $J=4.4,11.3 \mathrm{~Hz}, 1 \mathrm{H}, \mathrm{H}-5), 3.44$ (dt, $J=6.1,11.1 \mathrm{~Hz}, 1 \mathrm{H}, \mathrm{H}-5$ '), 3.02 (dd, $J=$ $\left.5.1,6.3 \mathrm{~Hz}, 1 \mathrm{H},-\mathrm{CH}_{2} \underline{\mathrm{OH}}\right), 2.68(\mathrm{dt}, J=7.6,17.9 \mathrm{~Hz}, 1 \mathrm{H}, \mathrm{H}-7), 2.56(\mathrm{dt}, J=7.2,17.9 \mathrm{~Hz}, 1 \mathrm{H}, \mathrm{H}-$ 7'), 2.32 (dd, $J=6.3,14.2 \mathrm{~Hz}, 1 \mathrm{H}, \mathrm{H}-3), 2.03$ (dd, $J=7.6,14.2 \mathrm{~Hz}, 1 \mathrm{H}, \mathrm{H}-3$ ') , 1.50-1.57 (m, 2H, H-8), 1.25-1.31 (m, 10H, H-9, H-10, H-11, H-12, H-13), 0.89 (t, $J=7.0 \mathrm{~Hz}, 3 \mathrm{H}, \mathrm{H}-14)$; $\delta_{\mathrm{C}}(100$ MHz, MeCN-d 3 ) 210.92 (C-6), 174.68 (C-1), 84.01 (C-2), 65.03 (C-5), 53.84 (C-4), 37.91 (C-7), 35.61 (C-3), 32.59, 30.09, 29.90, 29.79, 24.19 (C-8), 23.38 (C-9, C-10, C-11, C-12, C-13), 14.38 
$(\mathrm{C}-14) ; m / z(\mathrm{ESI}+) 294.30\left([\mathrm{M}+\mathrm{Na}]^{+}, 85 \%\right)$; HRMS $(\mathrm{ESI}+)$ calculated for $\mathrm{C}_{14} \mathrm{H}_{26} \mathrm{NO}_{4}\left([\mathrm{M}+\mathrm{H}]^{+}\right)$ 272.1856, found 272.1862 .

\section{(2S, 4R)-2-(Butylcarbonyl)-2-hydroxy-4-hydroxymethyl-1-oxo-pyrrolidinone, (2S)-8d}

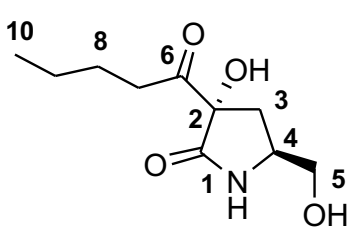

Scale of reaction: $37 \mathrm{mg}(0.122 \mathrm{mmol})$, product: $24 \mathrm{mg}, 91 \% ; \mathrm{R}_{f}=0.19$ (EtOAc); colourless oil; $[\alpha]_{\mathrm{D}}^{23}=+50.9(c=1.07$ in $\mathrm{MeOH}) ; v_{\max } / \mathrm{cm}^{-1}(\mathrm{film})$

3311 (br), $1716(\mathrm{~s}, \mathrm{C}=\mathrm{O}), 1690(\mathrm{~s}, \mathrm{C}=\mathrm{O}) ; \delta_{\mathrm{H}}\left(400 \mathrm{MHz}, \mathrm{MeCN}-\mathrm{d}_{3}\right) 6.81$ (br s, 1H, -NH-), 4.57 (s, 1H, -OH), 3.77-3.84 (m, 1H, H-4), 3.61 (ddd, J=4.1, 5.1, 11.2 Hz, 1H, H-5), $3.44\left(\mathrm{dt}, J=6.3,11.2 \mathrm{~Hz}, 1 \mathrm{H}, \mathrm{H}-5\right.$ ') $3.14\left(\mathrm{dd}, J=5.1,6.3 \mathrm{~Hz}, 1 \mathrm{H},-\mathrm{CH}_{2} \underline{\mathrm{OH}}\right), 2.69$ (dt, $J=7.3,17.9$ Hz, 1H, H-7), 2.58 (dt, $J=7.3,18.0 \mathrm{~Hz}, 1 \mathrm{H}, \mathrm{H}-7$ '), 2.31 (dd, $J=6.2,14.2 \mathrm{~Hz}, 1 \mathrm{H}, \mathrm{H}-3), 2.04$ (dd, $J$ $=7.6,14.2 \mathrm{~Hz}, 1 \mathrm{H}, \mathrm{H}-3$ ') $1.47-1.58(\mathrm{~m}, 2 \mathrm{H}, \mathrm{H}-8), 1.24-1.36(\mathrm{~m}, 2 \mathrm{H}, \mathrm{H}-9), 0.89$ (t, J = 7.4 Hz, 3H, H-10); $\delta_{\mathrm{C}}\left(100 \mathrm{MHz}, \mathrm{MeCN}-\mathrm{d}_{3}\right) 211.01$ (C-6), 174.98 (C-1), 84.07 (C-2), 65.04 (C-5), 53.92 (C-4), 37.67 (C-7), 35.64 (C-3), 26.28 (C-)8, 22.89 (C-9), 14.15 (C-10); m/z (ESI+) 238.23 ([M+Na] $]^{+}$ 40\%); HRMS (ESI+) calculated for $\mathrm{C}_{10} \mathrm{H}_{18} \mathrm{NO}_{4}\left([\mathrm{M}+\mathrm{H}]^{+}\right)$216.1230, found 216.1241.

\section{(2S, 4R)-2-Hydroxy-4-hydroxymethyl-2-(isobutylcarbonyl)-1-oxo-pyrrolidinone, (2S)-8e}

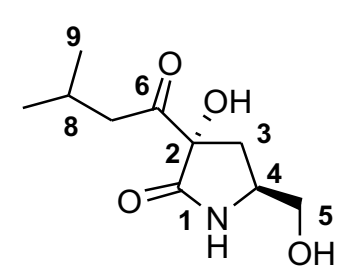

Scale of reaction: $5 \mathrm{mg}(0.0165 \mathrm{mmol})$, product: $3 \mathrm{mg}, 90 \% ; \mathrm{R}_{f}=0.16$ (EtOAc); colourless oil; $[\alpha]_{\mathrm{D}}^{23}=+50.9(c=0.70$ in $\mathrm{MeOH}) ; v_{\max } / \mathrm{cm}^{-1}$ (film) 3295 (br), $1716(\mathrm{~s}, \mathrm{C}=\mathrm{O}), 1690(\mathrm{~s}, \mathrm{C}=\mathrm{O}) ; \delta_{\mathrm{H}}\left(400 \mathrm{MHz}, \mathrm{MeCN}-\mathrm{d}_{3}\right) 6.66(\mathrm{br} \mathrm{s}$, 1H, -NH-), 4.46 (br s, 1H, -OH), 3.80-3.86 (m, 1H, H-4), 3.64 (dd, J=4.2, $11.2 \mathrm{~Hz}, 1 \mathrm{H}, \mathrm{H}-5), 3.47$ (dd, $J=6.2,11.2 \mathrm{~Hz}, 1 \mathrm{H}, \mathrm{H}-5$ '), 3.12 (br s, 1H, -OH), 2.62 (dd, $J=6.7,17.7 \mathrm{~Hz}, 1 \mathrm{H}, \mathrm{H}-7$ ), 2.49 (dd, $J=6.8,17.7 \mathrm{~Hz}, 1 \mathrm{H}, \mathrm{H}_{-1}$ '), 2.34 (dd, $\left.J=6.3,14.2 \mathrm{~Hz}, 1 \mathrm{H}, \mathrm{H}-3\right), 2.11-2.17$ (m, 1H, H-8, partially obscured by water signal), 2.06 (dd, $J=6.3,14.2 \mathrm{~Hz}, 1 \mathrm{H}, \mathrm{H}-3$ '), 0.93 (d, $J=6.7 \mathrm{~Hz}, 3 \mathrm{H}$, H-9), 0.92 (d, J=6.7 Hz, 3H, H-9); $\delta_{\mathrm{C}}\left(100 \mathrm{MHz},{\left.\mathrm{MeCN}-\mathrm{d}_{3}\right)}^{2} 10.39\right.$ (C-6), 174.61 (C-1), 84.04 (C2), 65.05 (C-5), 53.83 (C-4), 46.66 (C-7), 35.52 (C-3), 24.76 (C-8), 22.74, 22.67 (C-9); m/z (ESI-) 
$214.22\left([\mathrm{M}-\mathrm{H}]^{-}, 55 \%\right)$; HRMS (ESI+) calculated for $\mathrm{C}_{10} \mathrm{H}_{17} \mathrm{NNaO}_{4}\left([\mathrm{M}+\mathrm{Na}]^{+}\right) 238.1050$, found 238.1057.

(2S, 4R)-2-Hydroxy-4-hydroxymethyl-2-(phenylacetylenecarbonyl)-1-oxo-pyrrolidinone, $(2 S)$ 8f

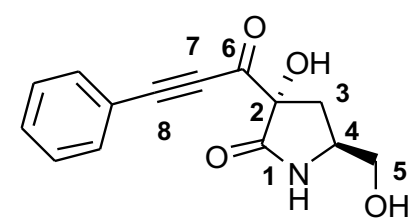

Scale of reaction: $82 \mathrm{mg}(0.236 \mathrm{mmol})$, product: $52 \mathrm{mg}, 85 \%$; $\mathrm{R}_{f}=0.27$ $\left(\right.$ EtOAc); white solid, $\mathrm{mp}=158-160^{\circ} \mathrm{C} ;[\alpha]_{\mathrm{D}}^{23}=-25.0(c=1.13$ in $\mathrm{MeOH}) ;$ $v_{\max } / \mathrm{cm}^{-1}$ (film) 3259 (br), 2195 (s, alkyne), 1697 (s, C=O), 1678 (s, $\mathrm{C}=\mathrm{O}) ; \delta_{\mathrm{H}}\left(400 \mathrm{MHz}, \mathrm{MeCN}_{-} \mathrm{d}_{3}\right)$ 7.63-7.70 (m, 2H, PhH), 7.53-7.60 (m, 1H, PhH), 7.44-7.51 (m, 2H, PhH), 6.78 (br s, 1H, -NH-), 4.61 (br s, 1H, -OH), 3.85-3.91 (m, 1H, H-4), 3.70 (ddd, J=4.1, 5.0, 11.3 Hz, 1H, H-5), 3.52 (dt, $J=6.3,11.3 \mathrm{~Hz}, 1 \mathrm{H}, \mathrm{H}-5$ '), 3.13 (dd, $J=5.1,6.4 \mathrm{~Hz}, 1 \mathrm{H},-$ $\mathrm{CH}_{2} \mathrm{OH}$ ), 2.69 (ddd, $\left.J=1.0,6.7,14.3 \mathrm{~Hz}, 1 \mathrm{H}, \mathrm{H}-3\right), 2.19$ (ddd, $J=0.8,7.3,14.3 \mathrm{~Hz}, 1 \mathrm{H}, \mathrm{H}-3$ '); $\delta_{\mathrm{C}}$ (100 MHz, MeCN-d 3 ) 187.33 (C-6), 173.62 (C-1), 134.24, 132.66, 130.00, 120.00 (PhC), 97.35 (C8), 85.92 (C-7), 85.51 (C-2), 65.03 (C-5), 54.06 (C-4), 35.74 (C-3); m/z (ESI-) 258.20 ([M-H]', 70\%); HRMS (ESI+) calculated for $\mathrm{C}_{14} \mathrm{H}_{14} \mathrm{NO}_{4}\left([\mathrm{M}+\mathrm{H}]^{+}\right)$260.0917, found 260.0911.

(2S, 4R)-2-Hydroxy-4-hydroxymethyl-2-(butylacetylenecarbonyl)-1-oxo-pyrrolidinone, (2S)-

$8 g$

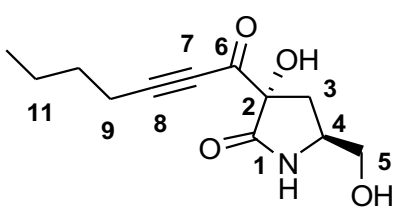

Scale of reaction: $87 \mathrm{mg}(0.266 \mathrm{mmol})$, product: $59 \mathrm{mg}, 93 \%$; $\mathrm{R}_{f}=0.29$ $($ EtOAc $)$; colourless crystals, $\mathrm{mp}=120-122^{\circ} \mathrm{C} ;[\alpha]_{\mathrm{D}}^{23}=+18.5(c=1.14$ in

$\mathrm{MeOH}) ; v_{\max } / \mathrm{cm}^{-1}$ (film) 3328 (peak with broad base), 2209 (s, alkyne), 1698 (s, C=O), 1686 (s, $\mathrm{C}=\mathrm{O}) ; \delta_{\mathrm{H}}\left(400 \mathrm{MHz}, \mathrm{MeCN}-\mathrm{d}_{3}\right) 6.71$ (br s, 1H, -NH-), 4.45 (br s, 1H, -OH), 3.76-3.88 (m, 1H, H4), $3.63(\mathrm{dt}, J=4.3,11.0 \mathrm{~Hz}, 1 \mathrm{H}, \mathrm{H}-5), 3.47$ (dt, $J=6.1,10.9 \mathrm{~Hz}, 1 \mathrm{H}, \mathrm{H}-5$ ') 3.05 (t, $J=5.6 \mathrm{~Hz}$, $\left.1 \mathrm{H},-\mathrm{CH}_{2} \underline{\mathrm{OH}}\right), 2.53(\mathrm{dd}, J=6.8,14.3 \mathrm{~Hz}, 1 \mathrm{H}, \mathrm{H}-3), 2.47$ (t, $\left.J=7.0 \mathrm{~Hz}, 2 \mathrm{H}, \mathrm{H}-9\right), 2.12(\mathrm{dd}, J=7.3$, $14.3 \mathrm{~Hz}, 1 \mathrm{H}, \mathrm{H}-3$ ') , 1.52-1.62 (m, 2H, H-10), 1.37-1.46 (m, 2H, H-11), 0.92 (t, J= 7.3 Hz, 3H, H12); $\delta_{\mathrm{C}}\left(100 \mathrm{MHz}, \mathrm{MeCN}_{-} \mathrm{d}_{3}\right) 187.27$ (C-6), 173.54 (C-1), 102.59 (C-8), 85.30 (C-2), 78.59 (C-7), 
65.48 (C-5), 54.04 (C-4), 35.88 (C-3), 30.26 (C-10), 22.67 (C-11), 19.34 (C-9), 13.74 (C-12); m/z $(\mathrm{ESI}+) 262.22\left([\mathrm{M}+\mathrm{Na}]^{+}, 100 \%\right)$; HRMS (ESI+) calculated for $\mathrm{C}_{12} \mathrm{H}_{18} \mathrm{NO}_{4}\left([\mathrm{M}+\mathrm{H}]^{+}\right) 240.1230$, found 240.1241 .

\section{Ru-catalysed dihydroxylation}

To a vigorously stirred solution of 9 (662 mg, $2.297 \mathrm{mmol}, 1.0$ eq.) in EtOAc/MeCN (1:1) (10 mL) at $0^{\circ} \mathrm{C}$ was added a solution of $\mathrm{RuCl}_{3} \cdot \mathrm{xH}_{2} \mathrm{O}(33 \mathrm{mg}, 0.161 \mathrm{mmol}, 0.07$ eq. $)$ and $\mathrm{NaIO}_{4}(737 \mathrm{mg}$, $3.45 \mathrm{mmol}, 1.5$ eq.) in distilled water $(5 \mathrm{~mL})$. The biphasic mixture was stirred vigorously for 20 min and quenched with sat. aq. $\mathrm{Na}_{2} \mathrm{~S}_{2} \mathrm{O}_{3}$ and extracted with EtOAc. The combined organic layers was dried with anhydrous $\mathrm{MgSO}_{4}$, filtered and the solvents removed in vacuo to give the crude. Purification via silica gel flash column chromatography (Eluent: 10\% to 80\% EtOAc/PE) gave diol $10(513 \mathrm{mg}, 69 \%)$ as a mixture diastereomers, and tetramic acid $\mathbf{1 1}(51 \mathrm{mg}, 7 \%)$ as a single diastereomer.

\section{$(2 R, \quad 5 S, \quad 6 R, \quad 7 R)-1-A z a-6,7-d i h y d r o x y-7-(N, O$-dimethylcarbamoyl)-3-oxa-8-oxo-2-phenyl-} bicyclo[3.3.0]octane, 10

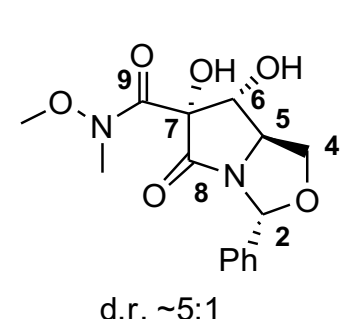

$\mathrm{R}_{f}=0.49$ (EtOAc); colourless oil; $v_{\max } / \mathrm{cm}^{-1}$ (film) $3386(\mathrm{br},-\mathrm{OH}), 1716(\mathrm{~s}$, $\mathrm{C}=\mathrm{O}), 1659(\mathrm{~s}, \mathrm{C}=\mathrm{O})$; Major diastereomer (cis-diol on exo face) $\delta_{\mathrm{H}}(400$ $\left.\mathrm{MHz}, \mathrm{MeCN}-\mathrm{d}_{3}\right)$ 7.34-7.51 (m, 5H, PhH), 6.21 (s, 1H, H-2), 4.81 (br s, 1H, d.r. $\sim 5: 1$ OH C-7), 4.45 (d, $J=5.1 \mathrm{~Hz}, 1 \mathrm{H}, \mathrm{H}-6), 4.38(\mathrm{ddd}, J=0.6,6.5,8.5 \mathrm{~Hz}, 1 \mathrm{H}, \mathrm{H}-$ 4exo), 4.00 (ddd, $J=5.1,6.5,7.8 \mathrm{~Hz}, 1 \mathrm{H}, \mathrm{H}-5), 3.75$ (t, $J=8.0 \mathrm{~Hz}, 1 \mathrm{H}, \mathrm{H}-4$ 'endo), 3.68 (s, 3H, OMe), 3.62 (br s, 1H, -OH C-6) 3.29 (s, 3H, -NMe); $\delta_{\mathrm{C}}\left(100 \mathrm{Mhz}, \mathrm{MeCN}-\mathrm{d}_{3}\right) 171.55$ (C-8), 169.86 (C-9), 139.34, 129.95, 129.63, 127.15 (PhC), 88.00 (C-2), 83.12 (C-7), 73.71 (C-6), 71.22 (C-4), 66.29 (C-5), 61.17 (-OMe), $33.61(-\mathrm{NMe}) ; \mathrm{m} / z(\mathrm{ESI}+) 345.27\left([\mathrm{M}+\mathrm{Na}]^{+}, 100 \%\right)$; HRMS (ESI+) calculated for $\mathrm{C}_{15} \mathrm{H}_{18} \mathrm{~N}_{2} \mathrm{NaO}_{6}\left([\mathrm{M}+\mathrm{Na}]^{+}\right)$345.1057, found 345.1069. 
$(2 R$,

oxabicyclo[3.3.0]-octane, 11

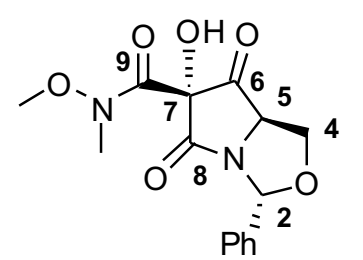

$\mathrm{R}_{f}=0.71($ EtOAc $) ;$ colourless crystals, $\mathrm{mp}=141-143^{\circ} \mathrm{C} ;[\alpha]_{\mathrm{D}}^{23}=+134.3(c=$

1.12 in $\mathrm{CH}_{2} \mathrm{Cl}_{2}$ ); $v_{\max } / \mathrm{cm}^{-1}$ (film) 3350 (br, $\left.-\mathrm{OH}\right), 1786$ (s, C=O), 1718 (s, $\mathrm{C}=\mathrm{O}), 1677(\mathrm{~s}, \mathrm{C}=\mathrm{O}) ; \delta_{\mathrm{H}}\left(400 \mathrm{MHz}, \mathrm{MeCN}^{-} \mathrm{d}_{3}\right)$ 7.36-7.53 (m, 5H, Ph), 6.69

(s, 1H, H-2), 5.34 (s, 1H, -OH), 4.74 (dd, $J=6.4,8.8 \mathrm{~Hz}, 1 \mathrm{H}, \mathrm{H}-5$ ), 4.22 (apparent t, $J=8.7 \mathrm{~Hz}$, 1H, H-4), 4.01 (dd, $J=6.4,8.5 \mathrm{~Hz}, 1 \mathrm{H}, \mathrm{H}-4$ '), 3.65 (s, 3H, -OMe), 3.25 (s, 3H, -NMe); $\delta_{\mathrm{C}}(100$ MHz, MeCN-d $\left.\mathrm{d}_{3}\right) 201.32$ (C-6), 171.46 (C-8), 166.54 (C-9), 138.11, 130.10, 129.77, 126.95 (PhC), 89.24 (C-2), 79.18 (C-7), 66.93 (C-4), 65.23 (C-5), 61.68 (-OMe), 33.58 (-NMe); m/z (ESI-) 319.57 ([M-H] $]^{-}, 100 \%$ ); HRMS (ESI-) calculated for $\mathrm{C}_{15} \mathrm{H}_{15} \mathrm{~N}_{2} \mathrm{O}_{6}\left([\mathrm{M}-\mathrm{H}]^{-}\right)$319.0936, found 319.0937.

\section{Grignard reaction of unprotected diol 10}

Tolyl magnesium bromide (3.4 eq.) was reacted with the Weinreb lactam 10 according to the General Procedure and the reaction mixture was stirred at $0^{\circ} \mathrm{C}$ for $30 \mathrm{~min}$, then r.t. for $1.5 \mathrm{~h}$. Each of the exo- and endo-diastereomers of $\mathbf{9 7 a / 1 2 a}$ was isolated (7\% yield each).

$(2 R, \quad 5 S, \quad 6 R, \quad 7 S)-1-A z a-6,7-d i h y d r o x y-7-(4$ 'methylphenylcarbonyl)-3-oxa-8-oxo-2-phenylbicyclo[3.3.0]octane, exo $-12 \mathrm{a}$

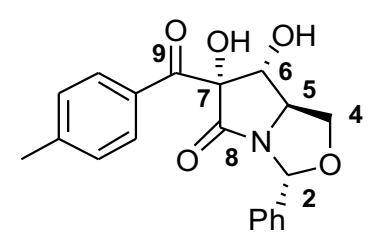

Scale of reaction: $52 \mathrm{mg}(0.161 \mathrm{mmol})$, product: $4 \mathrm{mg}, 7 \% ; \mathrm{R}_{f}=0.50(1: 1$ EtOAc:PE); white waxy oil; $[\alpha]_{\mathrm{D}}^{23}=+120.0\left(c=0.70\right.$ in $\left.\mathrm{CH}_{2} \mathrm{Cl}_{2}\right) ; v_{\max } / \mathrm{cm}^{-1}$ (film) 3359 (br, -OH), 1728 (s, C=O), 1669 (s, C=O); $\delta_{\mathrm{H}}(400 \mathrm{MHz}, \mathrm{MeCN}-$ d $\mathrm{d}_{3} 7.80(\mathrm{~d}, J=8.0 \mathrm{~Hz}, 2 \mathrm{H}, \mathrm{ArH}), 7.39-7.53(\mathrm{~m}, 5 \mathrm{H}, \mathrm{PhH}), 7.36(\mathrm{~d}, J=8.0 \mathrm{~Hz}, 2 \mathrm{H}, \mathrm{ArH}), 6.31(\mathrm{~s}$, 1H, H-2), 4.96 (br s, 1H, -OH), 4.72 (d, $J=5.9 \mathrm{~Hz}, 1 \mathrm{H}, \mathrm{H}-6), 4.39$ (ddd, $J=0.6,6.4,8.7 \mathrm{~Hz}, 1 \mathrm{H}$, H-4exo), 4.17 (dt, $J=6.1,7.2 \mathrm{~Hz}, 1 \mathrm{H}, \mathrm{H}-5), 3.95$ (dd, $J=7.3,8.7 \mathrm{~Hz}, 1 \mathrm{H}, \mathrm{H}-4$ endo), 3.88 (br s, 1H, -OH), 2.43 (s, 3H, Ar- $\underline{\mathrm{Me}}) ; \delta_{\mathrm{C}}\left(100 \mathrm{MHz}, \mathrm{MeCN}_{-} \mathrm{d}_{3}\right) 198.03$ (C-9), 172.51 (C-8), 146.04, 139.03, 132.19, 130.97, 130.20, 130.07, 129.66, 127.29 (ArC), 88.96 (C-7), 88.11 (C-2), 76.31 (C-6), 70.93 
(C-4), 65.07 (C-5), 21.77 (Ar-Me); m/z (ESI+) $376.27\left([\mathrm{M}+\mathrm{Na}]^{+}, 35 \%\right)$; HRMS (ESI+) calculated for $\mathrm{C}_{20} \mathrm{H}_{19} \mathrm{NNaO}_{5}\left([\mathrm{M}+\mathrm{Na}]^{+}\right)$376.1155, found 376.1140 .

$(2 R, \quad 5 S, \quad 6 S, \quad 7 S)-1-A z a-6,7-d i h y d r o x y-7-(4$ 'methylphenylcarbonyl)-3-oxa-8-oxo-2-phenylbicyclo[3.3.0]octane, endo -12 a

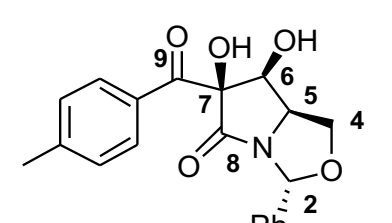

Product: $4 \mathrm{mg}, 7 \%$; $\mathrm{R}_{f}=0.38\left(1: 1\right.$ EtOAc:PE); white solid, $\mathrm{mp}=163^{\circ} \mathrm{C}$ decomposes to brown liquid; $[\alpha]_{\mathrm{D}}^{23}=+138.9\left(c=0.70\right.$ in $\left.\mathrm{CH}_{2} \mathrm{Cl}_{2}\right) ; v_{\max } / \mathrm{cm}^{-1}$ (film) 3362 (br, -OH), 1709 (s, C=O), 1669 (s, C=O); $\delta_{\mathrm{H}}(400 \mathrm{MHz}, \mathrm{MeCN}$ $\left.\mathrm{d}_{3}\right) 8.15(\mathrm{~d}, J=8.0 \mathrm{~Hz}, 2 \mathrm{H}, \mathrm{ArH}), 7.33-7.39(\mathrm{~m}, 5 \mathrm{H}, \mathrm{PhH}), 7.31(\mathrm{~d}, J=8.0 \mathrm{~Hz}, 2 \mathrm{H}, \operatorname{ArH}), 6.22(\mathrm{~s}$, 1H, H-2), 4.69 (d, $J=4.0 \mathrm{~Hz}, 1 \mathrm{H}, \mathrm{H}-6), 4.37$ (dt, $J=4.0,7.0 \mathrm{~Hz}, 1 \mathrm{H}, \mathrm{H}-5), 4.17$ (dd, $J=6.8,8.5$ Hz, 1H, H-4endo), 4.07 (dd, $J=7.2,8.4 \mathrm{~Hz}, 1 \mathrm{H}, \mathrm{H}-4$ exo), 2.40 (s, 3H, Ar-Me); $\delta_{\mathrm{C}}(100 \mathrm{MHz}$, MeCN-d 3 ) 196.26 (C-9), 173.30 (C-8), 145.86, 139.27, 133.06, 131.45, 129.88, 129.84, 129.55, 127.14 (ArC), 90.35 (C-7), 88.08 (C-2), 72.79 (C-6), 66.02 (C-4), 61.42 (C-5), 21.77 (Ar-Me); m/z $(\mathrm{ESI}+) 376.35\left([\mathrm{M}+\mathrm{Na}]^{+}, 50 \%\right)$; HRMS $(\mathrm{ESI}+)$ calculated for $\mathrm{C}_{20} \mathrm{H}_{19} \mathrm{NNaO}_{5}\left([\mathrm{M}+\mathrm{Na}]^{+}\right) 376.1155$, found 376.1141 .

$(2 R, 5 S, 6 R, 7 R)-1-A z a-6,7-(i s o p r o p y l i d e n e) d i o x y-7-(N, O$-dimethylcarbamoyl)-3-oxa-8-oxo-2phenyl-bicyclo[3.3.0]octane, 13

To a solution of $\mathbf{1 0}(176 \mathrm{mg}, 0.546 \mathrm{mmol}, 1.0$ eq.) in acetone ( $8 \mathrm{ml})$, were added 2,2dimethoxypropane (0.34 mL, $2.730 \mathrm{mmol}, 5$ eq.), and $p$-Ts $\mathrm{OH} \cdot \mathrm{H}_{2} \mathrm{O}$ (4 mg, $0.0218 \mathrm{mmol}, 0.04$ eq.) at r.t.. The mixture was stirred at $55^{\circ} \mathrm{C}$ for $6 \mathrm{~h}$. The reaction solvent was evaporated give the crude. Purification via silica gel flash column chromatography (Eluent: 10\% to 50\% EtOAc/PE) gave the

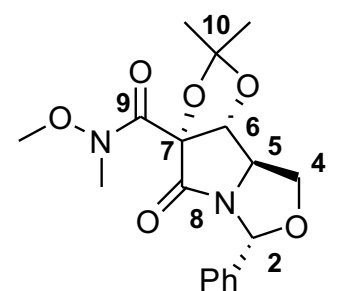

d.r. $\sim 1: 0.5$ acetonide 13 as a colourless oil (86 mg, 44\%), and unreacted 10 was recovered $(33 \mathrm{mg}$, d.r. $=1: 0.5) . \mathrm{R}_{f}=0.35(1: 1 \mathrm{EtOAc} / \mathrm{PE})$; colourless oil; $v_{\max } / \mathrm{cm}^{-1}$ (film) $1721(\mathrm{~s}, \mathrm{C}=\mathrm{O}), 1687(\mathrm{~s}, \mathrm{C}=\mathrm{O}) ; \delta_{\mathrm{H}}\left(400 \mathrm{MHz}, \mathrm{MeCN}-\mathrm{d}_{3}, 303 \mathrm{~K}\right)$ 7.347.50 (m, 5H, PhH), 6.33 (br s, 0.7H, H-2), 6.16 (s, 0.3H, H-2), 5.03 (br s, 0.7H, 
H-6), 4.95 (d, $J=4.9 \mathrm{~Hz}, 0.3 \mathrm{H}, \mathrm{H}-6), 4.33$ (t, $J=7.8 \mathrm{~Hz}, 1 \mathrm{H}, \mathrm{H}-4), 4.20$ (dd, $J=6.7,8.1 \mathrm{~Hz}, 0.3 \mathrm{H}$, H-4'), 4.05-4.15 (m, 1H, H-5), 3.69 (t, J=8.3 Hz, 0.7H, H-4'), 3.64 (s, 2H, -OMe), 3.43 (br s, 1H, OMe), 3.24 (br s, 3H, -NMe), 1.51 (s, 3H, -CMe), 1.44 (s, 2H, -CMe), 1.37 (s, 1H, -CMe); $\delta_{\mathrm{C}}(100$ MHz, MeCN-d 3 , 303K) 172.26 (v br, C-8), 168.39 (v br, C-9), 139.66, 139.43, 129.97, 129.82, 129.71, 129.67, 129.56, 127.28, 126.78 (PhC), 115.43 (C-10), 92.00 (C-7), 88.42, 88.34 (C-2), 82.76, 78.92 (br, C-6), 69.50, 66.58 (C-4), 66.10 (br, C-5), 60.73, 60.00 (-OMe), 33.66 (v br, $\mathrm{NMe}$ ), 28.35, 27.88, 27.30, 25.79 (Me of acetonide); $\delta_{\mathrm{H}}\left(400 \mathrm{MHz}, \mathrm{MeCN}-\mathrm{d}_{3}, 338 \mathrm{~K}\right)$ 7.34-7.51 (m, 5H, PhH), 6.33 (s, 0.7H, H-2), 6.17 (s, 0.3H, H-2), 5.03 (d, J=1.6 Hz, 0.7H, H-6), 4.95 (d, J= 4.8 Hz, 0.3H, H-6), 4.33 (t, $J=7.7$ Hz, 1H, H-4), 4.21 (dd, $J=6.8,8.3$ Hz, 0.3H, H-4'), 4.05-4.14 (m, 1H, H-5), 3.72 (t, $J=8.3 \mathrm{~Hz}, 0.7 \mathrm{H}, \mathrm{H}-4$ '), 3.66 (s, 2H, -OMe), 3.50 (s, 1H, -OMe), 3.28 (s, 3H, $\mathrm{NMe}), 1.52(\mathrm{~s}, 3 \mathrm{H},-\mathrm{CMe}), 1.46(\mathrm{~s}, 2 \mathrm{H},-\mathrm{CMe}), 1.41(\mathrm{~s}, 1 \mathrm{H},-\mathrm{CMe}) ; \delta_{\mathrm{C}}\left(100 \mathrm{MHz}, \mathrm{MeCN}-\mathrm{d}_{3}, 338 \mathrm{~K}\right)$ 173.07, 170.97 (C-8), 168.63 (C-9), 139.98, 139.74, 130.10, 129.97, 129.88, 129.83, 129.73, 127.44, 126.99 (PhC), 117.43, 115.75 (C-10), 93.79, 92.34 (C-7), 88.83, 88.67 (C-2), 82.89, 79.47 (C-6), 69.65, 66.74 (C-4), 66.23, 61.83 (C-5), 61.01, 60.16 (-OMe), 34.31(v br, -NMe), 28.46, 27.82, 27.61, $26.15\left(\mathrm{Me}\right.$ of acetonide); $\mathrm{m} / \mathrm{z}(\mathrm{ESI}+) 385.23\left([\mathrm{M}+\mathrm{Na}]^{+}, 60 \%\right)$; HRMS (ESI+) calculated for $\mathrm{C}_{18} \mathrm{H}_{22} \mathrm{~N}_{2} \mathrm{NaO}_{6}\left([\mathrm{M}+\mathrm{Na}]^{+}\right)$385.1370, found 385.1387 .

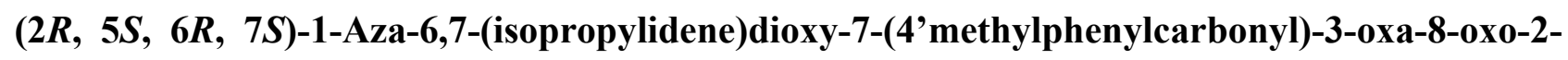
phenyl-bicyclo[3.3.0]octane, 14

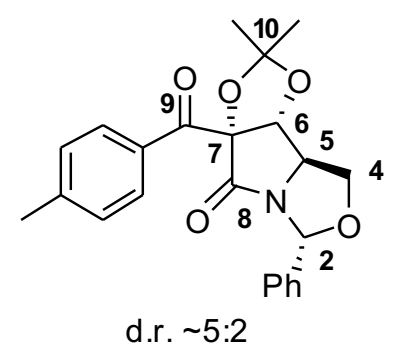

Tolyl magnesium bromide and the Grignard reaction were synthesised and carried out as described on p. 165. 1.3 eq. of the Grignard reagent was used and the reaction mixture was stirred at $0^{\circ} \mathrm{C}$ to r.t. over $1 \mathrm{~h}$. Scale of reaction: $70 \mathrm{mg}(0.198 \mathrm{mmol})$, product: $48 \mathrm{mg}, 63 \%$; $\mathrm{R}_{f}=0.45(30 \%$ EtOAc/PE); colourless oil; $v_{\max } / \mathrm{cm}^{-1}\left(\right.$ film) $1719(\mathrm{~s}, \mathrm{C}=\mathrm{O}), 1666(\mathrm{~s}, \mathrm{C}=\mathrm{O})$; Major diastereomer: $\delta_{\mathrm{H}}$ $\left(400 \mathrm{MHz}, \mathrm{MeCN}-\mathrm{d}_{3}\right) 8.13(\mathrm{~d}, J=8.3 \mathrm{~Hz}, 2 \mathrm{H}, \mathrm{ArH}), 7.31-7.48(\mathrm{~m}, 7 \mathrm{H}, \mathrm{PhH}$ and ArH), 6.15 (s, 1H, H-2), 5.14 (s, 1H, H-6), 4.42 (dd, $J=6.6,8.1 \mathrm{~Hz}, 1 \mathrm{H}, \mathrm{H}-4), 4.07-4.25$ (m, 1H, H-5), 3.80 (dd, $J=$ 
8.1, 10.0 Hz, 1H, H-4'), 2.43 (s, 3H, Ar- $\underline{\mathrm{Me}}$ ), 1.53 (s, 3H, C-Me), 1.13 (s, 3H, C-Me); $\delta_{\mathrm{C}}(100$ MHz, MeCN-d 3 ) 199.15 (C-9), 174.44 (C-8), 146.21, 139.64, 133.33, 131.90, 129.91, 129.64, 126.94 (ArC), 115.84 (C-10), 96.24 (C-7), 89.19 (C-2), 80.14 (C-6), 68.51 (C-4), 64.52 (C-5), 26.88, $25.73(2 \mathrm{x}$ C-Me $), 21.84(\mathrm{Ar}-\underline{\mathrm{Me}}) ; \mathrm{m} / \mathrm{z}(\mathrm{ESI}+) 416.39\left([\mathrm{M}+\mathrm{Na}]^{+}, 65 \%\right) ; \mathrm{HRMS}(\mathrm{ESI}+)$ calculated for $\mathrm{C}_{23} \mathrm{H}_{23} \mathrm{NNaO}_{5}\left([\mathrm{M}+\mathrm{Na}]^{+}\right)$416.1468, found 416.1453 .

\section{(2S, 3R, 4R)-2,3-(isopropylidene)dioxy-2-(4'-methylphenylcarbonyl)-4-hydroxymethyl-1-oxo-} pyrrolidinone, 15

Condition A: TFA ( $5 \mu \mathrm{L}, 0.635 \mathrm{mmol}, 10$ eq.) was added to a solution of 14 (25 mg, $0.0635 \mathrm{mmol}$, 1.0 eq.) in THF (1.5 mL with a drop of water) at r.t. and the reaction mixture stirred for $20 \mathrm{~h}$. Solvent was evaporated to give the crude, followed by purification via silica gel flash column chromatography(eluent: EtOAc/PE, 10\% to 50\%) gave $15(6.5 \mathrm{mg}, 34 \%$, d.r. 1:0.3 about the diol).

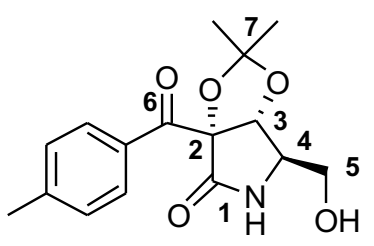

Condition B: A solution of 14 (17.5 mg, $0.0445 \mathrm{mmol}, 1.0$ eq.), $2 \mathrm{M}$ aq. $\mathrm{HCl}(88 \mu \mathrm{L}, 0.089 \mathrm{mmol}, 4$ eq. $)$ and $\mathrm{MeOH}(1 \mathrm{~mL})$ was stirred at r.t. for 6

h. Solvents were evaporated to give the crude, followed by purification via silica gel flash column chromatography (Eluent: EtOAc/PE, 10\% to 50\%) gave 15 (3 mg, 25\%, one diastereomer). $\mathrm{R}_{f}=0.21$ (1:1 EtOAc:PE); colourless oil; $[\alpha]_{\mathrm{D}}^{23}=-91.0\left(c=0.50\right.$ in $\left.\mathrm{CH}_{2} \mathrm{Cl}_{2}\right) ; v_{\max } /$ $\mathrm{cm}^{-1}$ (film) 3319 (br, $\left.-\mathrm{OH}\right), 1708(\mathrm{~s}, \mathrm{C}=\mathrm{O}), 1666(\mathrm{~s}, \mathrm{C}=\mathrm{O}) ; \delta_{\mathrm{H}}\left(400 \mathrm{MHz}, \mathrm{MeCN}-\mathrm{d}_{3}\right) 8.14(\mathrm{~d}, J=8.3$ Hz, 2H, ArH), 7.33 (d, J=8.0 Hz, 2H, ArH), 6.65 (br s, 1H, -NH-), 4.85 (s, 1H, H-3), 3.58-3.74 (m, 3H, H-4 and H-5), 2.42 (s, 3H, Ar-Me), 1.49 (s, 3H, CMe), 1.13 (s, 3H, ClMe); $\delta_{\mathrm{C}}(100 \mathrm{MHz}$, MeCN-d $\left.{ }_{3}\right) 199.03$ (C-6), 171.59 (C-1), 145.87, 133.57, 131.96, 129.74 (ArC), 114.45 (C-7), 92.89 (C-2), 82.19 (C-3), 63.51 (C-5), 60.45 (C-4), 26.92, 25.38 (2x -CMe), 21.81 (ArMe); m/z (ESI+) $328.26\left([\mathrm{M}+\mathrm{Na}]^{+}, 70 \%\right)$; HRMS $(\mathrm{ESI}+)$ calculated for $\mathrm{C}_{16} \mathrm{H}_{19} \mathrm{NNaO}_{5}\left([\mathrm{M}+\mathrm{Na}]^{+}\right) 328.1155$, found 328.1164. 
bicyclo[3.3.0]octane, 16

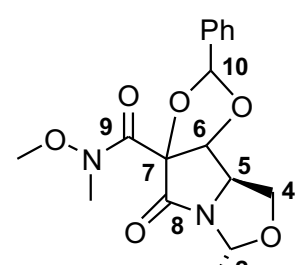

$\mathrm{Ph}^{\circ}$

To a solution of $\mathbf{1 0}(136 \mathrm{mg}, 0.422 \mathrm{mmol}, 1.0 \mathrm{eq}$.$) in toluene (6 \mathrm{~mL})$, were added benzaldehyde dimethyl acetal (158 $\mu \mathrm{L}, 1.055 \mathrm{mmol}, 2.5$ eq. $)$, and $p-\mathrm{TsOH}^{\circ} \mathrm{H}_{2} \mathrm{O}$

(3.2 mg, $0.0169 \mathrm{mmol}, 0.04$ eq.) at r.t.. The mixture was stirred at $65^{\circ} \mathrm{C}$ for 5.5 $\mathrm{h}$, and the solvents evaporated to give crude. Purification via silica gel flash column chromatography (Eluent: $10 \%$ to $45 \%$ EtOAc/PE) gave $16(96.1 \mathrm{mg}, 56 \%) . \mathrm{R}_{f}=0.47$ and 0.54 (2:1 EtOAc:PE); colourless oil; $v_{\max } / \mathrm{cm}^{-1}$ (film) $1718(\mathrm{~s}, \mathrm{C}=\mathrm{O}), 1683(\mathrm{~s}, \mathrm{C}=\mathrm{O}) ; \mathrm{m} / z(\mathrm{ESI}+)$ $411.37\left([\mathrm{M}+\mathrm{H}]^{+}, 100 \%\right)$; HRMS (ESI+) calculated for $\mathrm{C}_{22} \mathrm{H}_{22} \mathrm{~N}_{2} \mathrm{NaO}_{6}\left([\mathrm{M}+\mathrm{Na}]^{+}\right) 433.1370$, found 433.1384 .

(2R, 5S)-1-Aza-6,7-(phenylidene)dioxy-7-(4'methylphenylcarbonyl)-3-oxa-8-oxo-2-phenylbicyclo[3.3.0]octane, 17

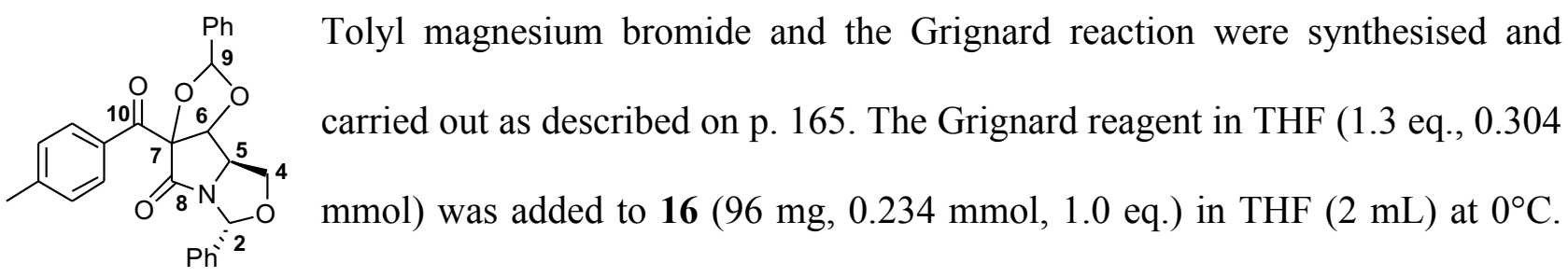

The reaction mixture was stirred at $0^{\circ} \mathrm{C}$ for 45 min. It was quenched with sat. aq. $\mathrm{NH}_{4} \mathrm{Cl}$ and extracted with EtOAc. The combined organic layers were dried with anhydrous $\mathrm{MgSO}_{4}$, filtered and the solvents removed in vacuo to give the crude product. Purification via silica gel flash column chromatography (Eluent: 5\% to 45\%, EtOAc/PE) gave $17(50 \mathrm{mg}, 48 \%) \cdot \mathrm{R}_{f}=0.46(2: 1 \mathrm{EtOAc:PE})$; colourless oil; $v_{\max } / \mathrm{cm}^{-1}\left(\right.$ film) $1718(\mathrm{~s}, \mathrm{C}=\mathrm{O}), 1666(\mathrm{~s}, \mathrm{C}=\mathrm{O}) ; m / z(\mathrm{ESI}+) 442.66\left([\mathrm{M}+\mathrm{H}]^{+}, 100 \%\right)$; HRMS (ESI+) calculated for $\mathrm{C}_{27} \mathrm{H}_{23} \mathrm{NNaO}_{5}\left([\mathrm{M}+\mathrm{Na}]^{+}\right) 464.1468$, found 464.1475.

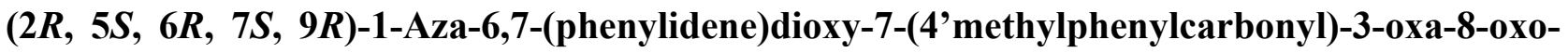
2-phenyl-bicyclo[3.3.0]octane, 17 


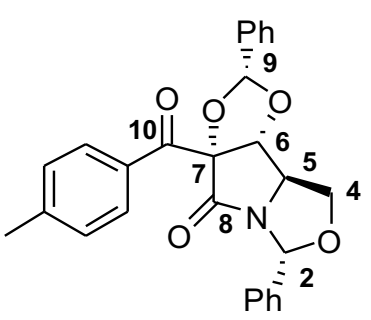

$\mathrm{R}_{f}=0.46\left(2: 1\right.$ EtOAc:PE); colourless oil; $[\alpha]_{\mathrm{D}}^{23}=+320.7\left(c=1.0\right.$ in $\left.\mathrm{CH}_{2} \mathrm{Cl}_{2}\right)$; $v_{\max } / \mathrm{cm}^{-1}($ film $) 1720(\mathrm{~s}, \mathrm{C}=\mathrm{O}), 1666(\mathrm{~s}, \mathrm{C}=\mathrm{O}) ; \delta_{\mathrm{H}}\left(400 \mathrm{MHz}, \mathrm{MeCN}-\mathrm{d}_{3}\right)$ $8.17(\mathrm{~d}, J=8.3 \mathrm{~Hz}, 2 \mathrm{H}, \mathrm{ArH}), 7.46-7.57$ (m, 5H, ArH), 7.29-7.45 (m, 7H, ArH), $6.31(\mathrm{~s}, 1 \mathrm{H}, \mathrm{H}-2), 6.13$ (s, 1H, H-9), 5.20 (d, $J=4.4 \mathrm{~Hz}, 1 \mathrm{H}, \mathrm{H}-6)$, $4.58(\mathrm{dt}, J=4.4,7.2 \mathrm{~Hz}, 1 \mathrm{H}, \mathrm{H}-5), 4.23(\mathrm{dd}, J=7.1,8.3 \mathrm{~Hz}, 1 \mathrm{H}, \mathrm{H}-4), 4.16(\mathrm{dd}, J=7.4,8.6 \mathrm{~Hz}$, 1H, H-4'), 2.40 (s, 3H, Ar-Me); $\delta_{\mathrm{C}}\left(100 \mathrm{MHz}, \mathrm{MeCN}_{-} \mathrm{d}_{3}\right)$ 195.14 (C-10), 171.38 (C-8), 146.63, 138.80, 136.79, 133.06, 131.70, 131.36, 130.09, 130.04, 139.66, 128.27, 127.18 (ArC), 108.25 (C9), 98.15 (C-7), 88.36 (C-2), 81.52 (C-6), 66.21 (C-4), 59.52 (C-5), 21.87 (Ar-Me); m/z (ESI+) $442.64\left([\mathrm{M}+\mathrm{H}]^{+}, 100 \%\right)$; HRMS (ESI+) calculated for $\mathrm{C}_{27} \mathrm{H}_{23} \mathrm{NNaO}_{5}\left([\mathrm{M}+\mathrm{Na}]^{+}\right) 464.1468$, found 464.1475.

(2S, 3R, 4R, 6S)-2,3-(phenylidene)dioxy-2-(4'-methylphenylcarbonyl)-4-hydroxymethyl-1-oxopyrrolidinone, 18

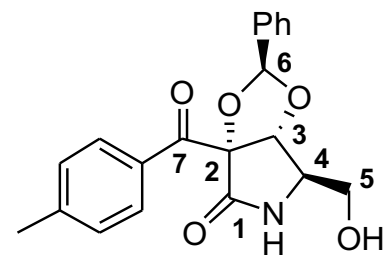

TFA (18 $\mu \mathrm{L}, 2.265 \mathrm{mmol}, 20$ eq.) was added to 17 (50 mg, $0.113 \mathrm{mmol}, 1$ eq.) in $\mathrm{CH}_{2} \mathrm{Cl}_{2}(1.5 \mathrm{~mL})$ and the resulting pale yellow solution was stirred at r.t. for $5.5 \mathrm{~h}$. The solvents were removed in vacuo to give the crude which was purified via silica gel column chromatography (Eluent: $10 \%$ to $60 \% \mathrm{EtOAc} / \mathrm{PE}$ ) to give a one fraction containing a diastereomeric mixture of $\mathbf{1 8}(13 \mathrm{mg})$ and one pure diastereomer of $\mathbf{1 8}$ $(5 \mathrm{mg}, 13 \%)$. One diastereomer of 17 was recovered $(16 \%) \cdot \mathrm{R}_{f}=0.13(1: 1 \mathrm{EtOAc}: \mathrm{PE})$; colourless oil; $[\alpha]_{\mathrm{D}}^{23}=+23.4\left(c=0.63\right.$ in $\left.\mathrm{CH}_{2} \mathrm{Cl}_{2}\right) ; v_{\max } / \mathrm{cm}^{-1}$ (film) $1714(\mathrm{~s}, \mathrm{C}=\mathrm{O}), 1669(\mathrm{~s}, \mathrm{C}=\mathrm{O}) ; \delta_{\mathrm{H}}(400$ MHz, MeCN-d $\left.{ }_{3}\right) 8.09$ (d, $\left.J=8.3 \mathrm{~Hz}, 2 \mathrm{H}, \mathrm{ArH}\right), 7.32-7.48(\mathrm{~m}, 5 \mathrm{H}, \mathrm{ArH}), 7.30(\mathrm{~d}, J=8.3 \mathrm{~Hz}, 2 \mathrm{H}$, ArH), 6.97 (br s, 1H, -NH-), 6.15 (s, 1H, H-6), 4.97 (s, 1H, H-3), 3.81 (tt, $J=1.2,6.4$ Hz, 1H, H-4), 3.60-3.72 (m, 2H, H-5 and H-5') $3.26(\mathrm{dt}, J=1.3,5.7 \mathrm{~Hz}, 1 \mathrm{H},-\mathrm{OH}), 2.39$ (s, 3H, Ar-Me); $\delta_{\mathrm{C}}(100$ MHz, MeCN-d ${ }_{3}$ ) 196.51 (C-7), 170.62 (C-1), 145.96, 136.52, 133.46, 131.60, 130.98, 129.81, 129.47, 127.84 (ArC), 106.05 (C-6), 92.92 (C-2), 82.05 (C-3), 63.61 (C-4), 61.26 (C-5), 21.79 (Ar- 
$\underline{\mathrm{Me}}) ; m / z(\mathrm{ESI}+) 376.56\left([\mathrm{M}+\mathrm{Na}]^{+}, 100 \%\right)$; HRMS $(\mathrm{ESI}+)$ calculated for $\mathrm{C}_{22} \mathrm{H}_{19} \mathrm{NNaO}_{5}\left([\mathrm{M}+\mathrm{Na}]^{+}\right)$ 376.1155 , found 376.1172 .

$(2 S, 3 R, 4 R, 6 R, 7 S)-2,3-($ phenylidene)dioxy-2-(hydroxymethyl-4'-methylphenylcarbonyl)-4hydroxymethyl-1-oxo-pyrrolidinone, 19

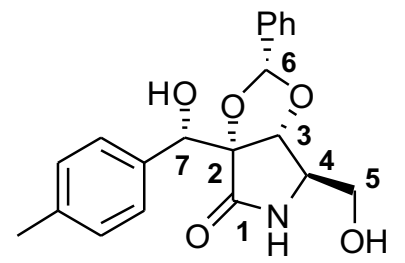

$10 \% \mathrm{Pd} / \mathrm{C}(1.5 \mathrm{mg})$ was added to a diastereomeric mixture of $18(13 \mathrm{mg}$, $0.0368 \mathrm{mmol})$ in EtOH $(3 \mathrm{~mL})$. The suspension was stirred at r.t. with bubbling of $\mathrm{H}_{2}$ (balloon) for $6 \mathrm{~h}$, afterwhich it was filtered over Celite and the residue rinsed with EtOAc. The solvent was evaporated to give the crude which was purified via silica gel flash column chromatography (Eluent: 10\% to 60\% EtOAc/PE) to give 19 (4 mg, 31\%). $\mathrm{R}_{f}=0.63\left(\right.$ EtOAc); fine white crystals, $\mathrm{mp}=156-158^{\circ} \mathrm{C} ;[\alpha]_{\mathrm{D}}^{23}=+26.0(c=0.68$ in $\mathrm{MeOH}) ; v_{\max } /$ $\mathrm{cm}^{-1}$ (film) 3329 (br), 1695 (s, C=O,); $\delta_{\mathrm{H}}\left(400 \mathrm{MHz}, \mathrm{MeCN}-\mathrm{d}_{3}\right)$ 7.39-7.45 (m, 5H, PhH), 7.33 (d, J $=8.1 \mathrm{~Hz}, 2 \mathrm{H}, \mathrm{ArH}), 7.19(\mathrm{~d}, J=7.9 \mathrm{~Hz}, 2 \mathrm{H}, \mathrm{ArH}), 6.37$ (br s, 1H, -NH-), $5.98(\mathrm{~s}, 1 \mathrm{H}, \mathrm{H}-6), 5.25$ (d, $J=3.3 \mathrm{~Hz}, 1 \mathrm{H}, \mathrm{H}-7), 4.55$ (s, 1H, H-3), 4.25 (d, $J=3.3 \mathrm{~Hz}, 1 \mathrm{H}, \mathrm{C} 7-\mathrm{OH}), 3.42-3.46$ (m, 1H, H-4), $2.86(\mathrm{t}, J=5.6 \mathrm{~Hz}, 1 \mathrm{H}, \mathrm{C} 5-\mathrm{OH}), 2.49$ (ddd, $J=5.5,6.9,10.9 \mathrm{~Hz}, 1 \mathrm{H}, \mathrm{H}-5), 2.43$ (ddd, $J=5.5,6.9$, $10.9 \mathrm{~Hz}, 1 \mathrm{H}, \mathrm{H}-5$ ') 2.34 (s, 3H, Ar-Me); $\delta_{\mathrm{C}}\left(100 \mathrm{MHz}, \mathrm{MeCN}-\mathrm{d}_{3}\right) 173.46$ (C-1), 138.96, 137.82, 136.23, 130.71, 129.78, 129.40, 128.17, 127.81 (ArC), 107.28 (C-6), 90.00 (C-2), 79.27 (C-3), 73.22 (C-7), 62.64 (C-5), 60.27 (C-4), 21.22 (Ar-Me); m/z (ESI+) 354.65 ([M-H]', 100\%); HRMS (ESI+) calculated for $\mathrm{C}_{20} \mathrm{H}_{21} \mathrm{NNaO}_{5}\left([\mathrm{M}+\mathrm{Na}]^{+}\right)$378.1312, found 378.1323.

\section{(2R, 5S)-1-Aza-6,7-(4'-methoxyphenylidene)dioxy-7-(N,O-dimethylcarbamoyl)-3-oxa-8-oxo-2-}

\section{phenyl-bicyclo[3.3.0]octane, 20}

To a solution of $\mathbf{1 0}$ (513 mg, $1.592 \mathrm{mmol} 1.0$ eq.) in toluene (12 mL), were added anisaldehyde dimethyl acetal ( $680 \mu \mathrm{L}, 3.98 \mathrm{mmol}, 2.5$ eq. $)$, and $p$ - $\mathrm{TsOH}^{\cdot} \mathrm{H}_{2} \mathrm{O}$ (12.2 mg, $0.064 \mathrm{mmol}, 0.04$ eq. $)$ at r.t. The mixture was stirred at $65^{\circ} \mathrm{C}$ for $5.5 \mathrm{~h}$, and the solvents evaporated to give crude. Purification via silica gel flash column chromatography (Eluent: 5\% to 40\% EtOAc/PE) gave 20 


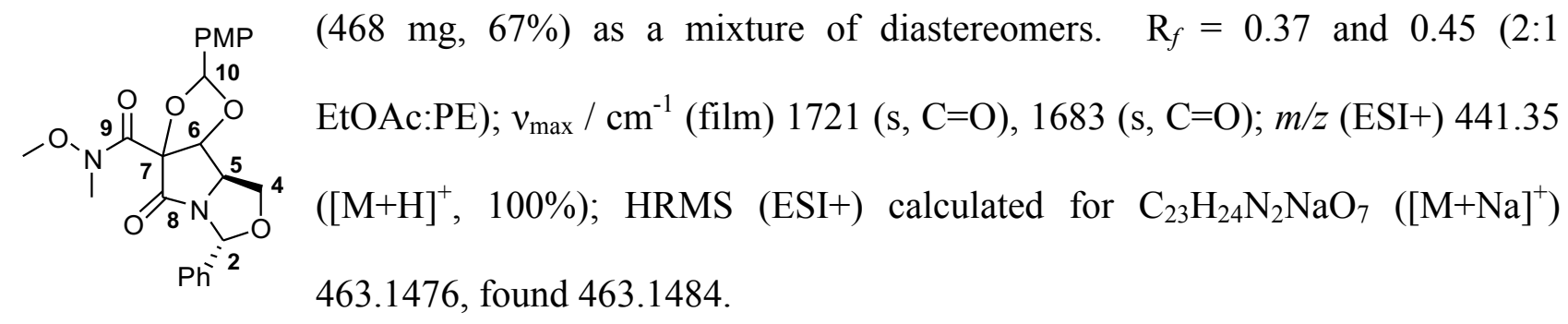

\section{General procedure for Grignard reaction of Weinreb amide 20}

The Grignard reagent in THF (1.3 eq. based on amount of 20 used) was added to 20 (1.0 eq.) in THF at $0^{\circ} \mathrm{C}$. The reaction was stirred at $0^{\circ} \mathrm{C}$ to r.t. over a period of $45 \mathrm{~min}$ to $1 \mathrm{~h}$, afterwhich it was quenched with sat. aq. $\mathrm{NH}_{4} \mathrm{Cl}$ and extracted with EtOAc. The combined organic layers were dried with anhydrous $\mathrm{MgSO}_{4}$, filtered and the solvents removed in vacuo to give the crude product. Purification via silica gel flash column chromatography (Eluent: EtOAc/PE) gave the respective product as mixture of diastereomers about the protected diol.

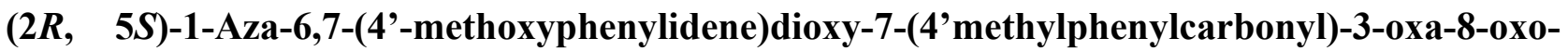
2-phenyl-bicyclo[3.3.0]octane, 21a

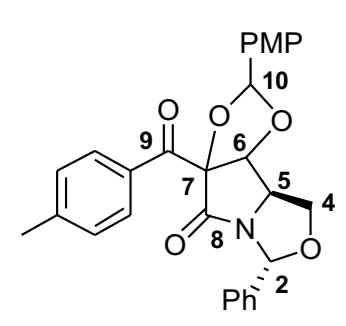

Scale of reaction: $77 \mathrm{mg}(0.175 \mathrm{mmol})$, product: $37 \mathrm{mg}, 45 \%$; $\mathrm{R}_{f}=0.53(30 \%$ EtOAc/PE); colourless oil; $v_{\max } / \mathrm{cm}^{-1}$ (film) $1718(\mathrm{~s}, \mathrm{C}=\mathrm{O}), 1667(\mathrm{~s}, \mathrm{C}=\mathrm{O}) ; \delta_{\mathrm{H}}$ $\left(400 \mathrm{MHz}, \mathrm{MeCN}_{-} \mathrm{d}_{3}\right) 8.16(\mathrm{~d}, J=8.2 \mathrm{~Hz}, \mathrm{Ar}-\mathrm{H}), 8.14(\mathrm{~d}, J=8.2 \mathrm{~Hz}, \mathrm{Ar}-\mathrm{H})$, $8.06(\mathrm{~d}, J=8.3 \mathrm{~Hz}, \operatorname{Ar}-\mathrm{H}), 7.51-7.56(\mathrm{~m}, \mathrm{ArH}), 7.00(\mathrm{~d}, J=8.8 \mathrm{~Hz}, \operatorname{Ar}-\mathrm{H})$,

$6.93(\mathrm{~d}, J=8.8 \mathrm{~Hz}, \mathrm{Ar}-\mathrm{H}), 6.88$ (d, $J=8.9 \mathrm{~Hz}, \mathrm{Ar}-\mathrm{H}), 6.32,6.23,6.17$ (3x s, H-2), 6.20, 6.08, 5.95 (3x s, H-10), 5.30 (d, $J=1.2$ Hz, H-6), 5.20 (s, H-6), 5.17 (d, $J=4.4$ Hz, H-6), 4.56 (dt, $J=4.4,7.2$ Hz, H-5), 4.50 (dd, $J=6.5,8.3$ Hz, H-4), 4.43 (dd, $J=6.6,8.1 \mathrm{~Hz}, \mathrm{H}-4), 4.31$ (ddd, $J=1.2,6.5,9.8$ Hz, H-5), 4.19-4.27 (m, H-4), 4.14 (dd, $J=7.4,8.6$ Hz, H-4), 3.83, 3.79, 3.76 (3x s, 3H, Ar-OMe), 2.42, 2.40, 2.39 (3x s, 3H, Ar-Me); $\delta_{\mathrm{C}}\left(100 \mathrm{MHz}, \mathrm{MeCN}_{-} \mathrm{d}_{3}\right)$ 198.47, 196.69, 195.22 (C-9), 173.60, 173.36, 171.52 (C-8), 162.31, 162.20, 162.17, 146.57, 146.43, 146.18, 139.50, 139.45, 138.79, $133.31,133.05,131.78,131.67,131.65,130.05,130.01,129.91,129.89,129.83,129.80,129.63$, $129.31,128.72,128.36,127.73,127.22,127.16,127.14,126.94,118.29,114.96,114.91,114.80$ 
(ArC), 108.22, 107.89, 106.57 (C-10), 97.98, 96.06, 95.75 (C-7), 89.45, 89.29, 88.32 (C-2), 81.33, 80.65, 80.20 (C-6), 69.21, 68.39, 66.17 (C-4), 64.77, 64.00, 59.48 (C-5), 56.11, 56.06, 56.03 (Ar-

OMe $), 21.85,21.81(\mathrm{Ar}-\underline{\mathrm{Me}}) ; \mathrm{m} / z(\mathrm{ESI}+) 494.33\left([\mathrm{M}+\mathrm{Na}]^{+}, 100 \%\right)$; HRMS (ESI+) calculated for $\mathrm{C}_{28} \mathrm{H}_{25} \mathrm{NNaO}_{6}\left([\mathrm{M}+\mathrm{Na}]^{+}\right)$495.1607, found 495.1588.

(2R, 5S)-1-Aza-6,7-(4'-methoxyphenylidene)dioxy-7-(4'methoxyphenylcarbonyl)-3-oxa-8-oxo2-phenyl-bicyclo[3.3.0]octane, 21b

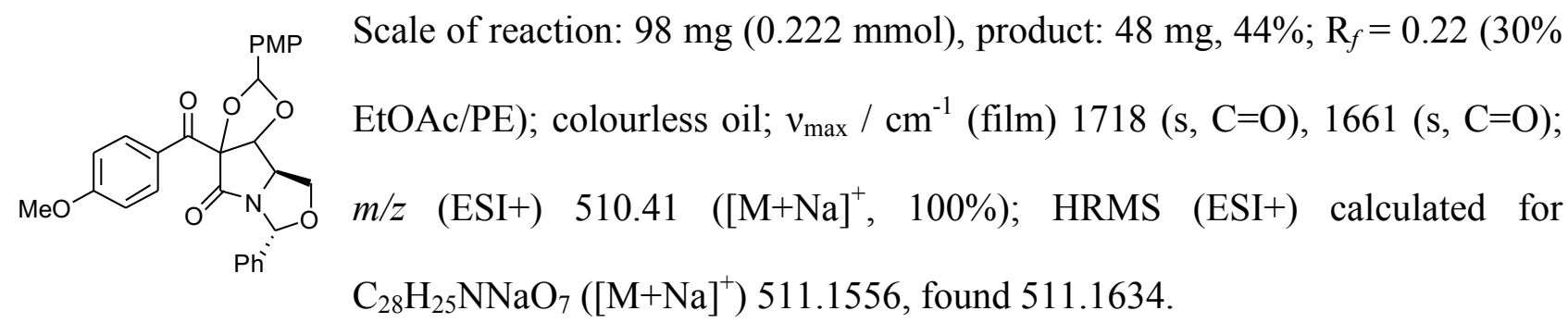

(2R, 5S)-1-Aza-6,7-(4'-methoxyphenylidene)dioxy-7-(octylcarbonyl)-3-oxa-8-oxo-2-phenylbicyclo[3.3.0]octane, 21c

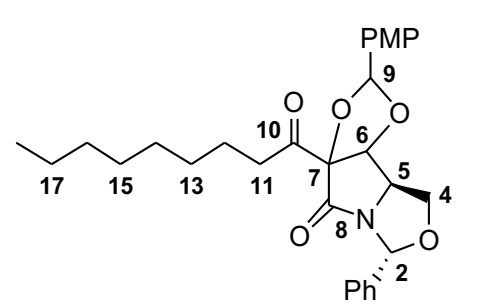

Scale of reaction: $128 \mathrm{mg}(0.291 \mathrm{mmol})$, product: $43 \mathrm{mg}, 30 \%$; $\mathrm{R}_{f}=$ $0.42(30 \% \mathrm{EtOAc} / \mathrm{PE})$; colourless oil; $v_{\max } / \mathrm{cm}^{-1}$ (film) $1726(\mathrm{~s}, \mathrm{C}=\mathrm{O})$, $1709(\mathrm{~s}, \mathrm{C}=\mathrm{O}) ; \mathrm{m} / \mathrm{z}(\mathrm{ESI}+) 494.48\left([\mathrm{M}+\mathrm{H}]^{+}, 100 \%\right) ; \operatorname{HRMS}(\mathrm{ESI}+)$ calculated for $\mathrm{C}_{29} \mathrm{H}_{36} \mathrm{NO}_{6}\left([\mathrm{M}+\mathrm{H}]^{+}\right)$494.2537, found 494.2543.

(2R, 5S)-1-Aza-7-(butylcarbonyl)-6,7-(4'-methoxyphenylidene)dioxy-3-oxa-8-oxo-2-phenylbicyclo[3.3.0]octane, 21d

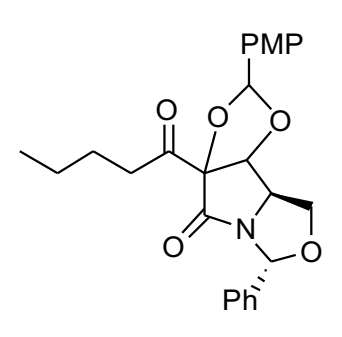

Scale of reaction: $135 \mathrm{mg}(0.307 \mathrm{mmol})$, product: $33 \mathrm{mg}, 19 \% ; \mathrm{R}_{f}=0.28(30 \%$ EtOAc/PE); colourless oil; $v_{\max } / \mathrm{cm}^{-1}$ (film) $1724(\mathrm{~s}, \mathrm{C}=\mathrm{O}), 1708$ (s, C=O); $m / z \quad(\mathrm{ESI}+) \quad 438.42\left([\mathrm{M}+\mathrm{H}]^{+}, \quad 100 \%\right) ; \quad \mathrm{HRMS} \quad(\mathrm{ESI}+)$ calculated for $\mathrm{C}_{25} \mathrm{H}_{27} \mathrm{NNaO}_{6}\left([\mathrm{M}+\mathrm{Na}]^{+}\right)$460.1731, found 460.1734 . 


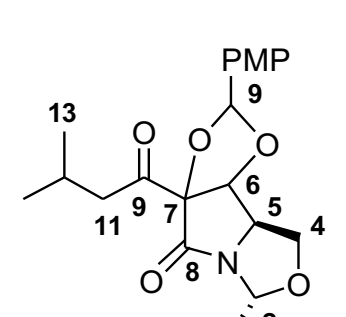

$\mathrm{Ph}^{2}$

Scale of reaction: $140 \mathrm{mg}(0.318 \mathrm{mmol})$, product: $37 \mathrm{mg}, 27 \% ; \mathrm{R}_{f}=0.33(30 \%$

EtOAc/PE); colourless oil; $v_{\max } / \mathrm{cm}^{-1}$ (film) 1707 (s, C=O, with shoulder towards longer wave number); $m / z(\mathrm{ESI}+) 438.39\left([\mathrm{M}+\mathrm{H}]^{+}, 25 \%\right)$; HRMS (ESI+) calculated for $\mathrm{C}_{25} \mathrm{H}_{27} \mathrm{NO}_{6}\left([\mathrm{M}+\mathrm{H}]^{+}\right)$438.1911, found 438.1927.

(2R, 5S)-1-Aza-6,7-(4'-methoxyphenylidene)dioxy-7-(phenylacetylenecarbonyl)-3-oxa-8-oxo-

2-phenyl-bicyclo[3.3.0]octane, $21 \mathrm{f}$

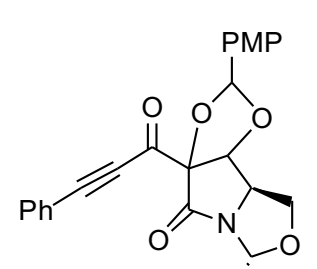

$\mathrm{Ph}^{\circ}$

Scale of reaction: $130 \mathrm{mg}(0.295 \mathrm{mmol})$, product: $72 \mathrm{mg}, 51 \% ; \mathrm{R}_{f}=0.68(1: 1$

EtOAc/PE); pale yellow oil; $v_{\max } / \mathrm{cm}^{-1}$ (film) 2192 (s, alkyne), 1722 (s, C=O),

$1661(\mathrm{~s}, \mathrm{C}=\mathrm{O}) ; \mathrm{m} / z(\mathrm{ESI}+) 482.48\left([\mathrm{M}+\mathrm{H}]^{+}, 55 \%\right)$; HRMS (ESI+) calculated for $\mathrm{C}_{29} \mathrm{H}_{23} \mathrm{NNaO}_{6}\left([\mathrm{M}+\mathrm{Na}]^{+}\right)$504.1418, found 504.1431.

\section{$(2 R, 5 S, 6 R, 7 S)$-1-Aza-7-(butylacetylenecarbonyl)-6,7-(4'-methoxyphenylidene)dioxy-3-oxa-}

\section{8-oxo-2-phenyl-bicyclo[3.3.0]octane, 21g}

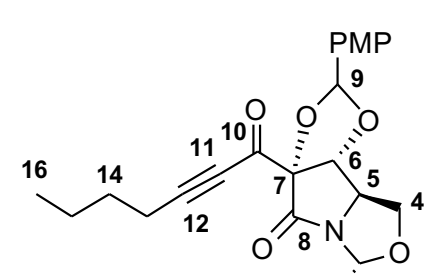

$\mathrm{Ph}^{\circ}$

d.r. $\sim 1: 1$ about C-9

Scale of reaction: $135 \mathrm{mg}(0.307 \mathrm{mmol})$, product: $48 \mathrm{mg}, 34 \% ; \mathrm{R}_{f}=0.22$ (30\% EtOAc/PE); colourless oil; $v_{\max } / \mathrm{cm}^{-1}$ (film) 2212 (s, alkyne), 1724 $(\mathrm{s}, \mathrm{C}=\mathrm{O}), 1665(\mathrm{~s}, \mathrm{C}=\mathrm{O}) ; \delta_{\mathrm{H}}\left(400 \mathrm{MHz}, \mathrm{MeCN}_{-} \mathrm{d}_{3}\right)$ 7.49-7.54 (m, 2H, ArH), 7.37-7.48 (m, 5H, PhH), 6.92-7.01 (m, 2H, ArH), 6.28 (s, 0.5H, H2), 6.25 (s, $0.5 \mathrm{H}, \mathrm{H}-2), 6.15$ (s, $0.5 \mathrm{H}, \mathrm{H}-9), 6.11$ (s, $0.5 \mathrm{H}, \mathrm{H}-9), 5.10$ (d, $J=1.4 \mathrm{~Hz}, 0.5 \mathrm{H}, \mathrm{H}-6), 4.97$ (s, 0.5H, H-6), 4.48 (dd, $J=6.5,8.4 \mathrm{~Hz}, 0.5 \mathrm{H}, \mathrm{H}-4), 4.40$ (dd, $J=6.7$, 8.2 Hz, 0.5H, H-4), 4.19-4.31 (m, 1H, H-5), 3.81 and 3.80 (s, 3H, Ar-OMe), 3.68 (ddd, J=4.6, 8.4, $9.7 \mathrm{~Hz}, 1 \mathrm{H}, \mathrm{H}-4), 2.53$ (dt, $J=7.0,15.1 \mathrm{~Hz}, 2 \mathrm{H}, \mathrm{H}-13), 1.52-1.66$ (m, 2H, H-14), 1.35-1.50 (m, 2H, H-15), 0.86-0.96 (m, 3H, H-16); $\delta_{\mathrm{C}}\left(100 \mathrm{MHz}, \mathrm{MeCN}_{-} \mathrm{d}_{3}\right)$ 184.23, 182.61 (C-10), 172.53, 172.40 (C-8), 162.29, 162.25 (ArC quart), 139.31, 139.26, 130.07, 129.98, 129.92, 129.65, 129.39, 127.94, 127.67, 127.21, 126.95, 114.92, 114.83 (ArC), 108.87, 106.87 (C-9), 104.32, 103.84 (C-12), 95.21, 94.91 (C-7), 89.35, 89.28 (C-2), 80.60, 80.46 (C-11), 79.64, 79.43 (C-6), 69.55, 68.60 (C-4), 64.83, 
64.17 (C-5), 56.08, 55.33 (Ar-OMe), 30.22, 30.19 (C-14), 22.68 (C-15), 19.48, 19.47 (C-13), 13.76, $13.75(\mathrm{C}-16) ; m / z(\mathrm{ESI}+) 462.38\left([\mathrm{M}+\mathrm{H}]^{+}, 15 \%\right)$; HRMS (ESI+) calculated for $\mathrm{C}_{27} \mathrm{H}_{28} \mathrm{NO}_{6}$ $\left([\mathrm{M}+\mathrm{H}]^{+}\right)$462.1911, found 462.1917 .

\section{General procedure for acidic oxazolidine hydrolysis to 2,3-dihydroxypyrrolidinones 22a-g}

TFA (20 eq.) was added to a solution of $21 \mathbf{a - g}$ in $\mathrm{CH}_{2} \mathrm{Cl}_{2}$ at r.t.. The reaction mixture was stirred at r.t. for 5-6.5 h and the solvents evaporated to give the crude. Purification via silica gel flash column chromatography (Eluent: $0 \%$ to $10 \% \quad \mathrm{MeOH} / \mathrm{EtOAc}$ ) gave the respective 2,3dihydroxypyrrolidinones.

\section{(2S, 3R, 4R)-2,3-Dihydroxy-2-(4'-methylphenylcarbonyl)-4-hydroxymethyl-1-oxo-}

pyrrolidinone, 22a

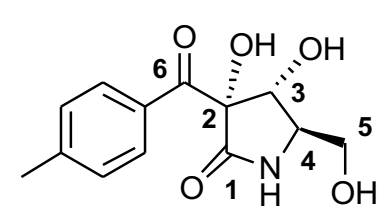

Scale of reaction: $35 \mathrm{mg}(0.0742 \mathrm{mmol})$, product: $9 \mathrm{mg}, 45 \% ; \mathrm{R}_{f}=0.23$

(EtOAc); white solid, $\mathrm{mp}=154-156^{\circ} \mathrm{C} ;[\alpha]_{\mathrm{D}}^{23}=-33.4(c=1.35$ in $\mathrm{MeOH}) ;$ $v_{\max } / \mathrm{cm}^{-1}$ (film) 3452 (br, -NH-), $1692(\mathrm{~s}, \mathrm{C}=\mathrm{O}), 1663(\mathrm{~s}, \mathrm{C}=\mathrm{O}) ; \delta_{\mathrm{H}}(400$ MHz, MeCN-d 3 ) 7.92 (d, J=8.3 Hz, 2H, ArH), 7.32 (d, J=8.3 Hz, 2H, ArH), 6.76 (br s, 1H, -NH), 4.85 (br s, $1 \mathrm{H},-\mathrm{OH}), 4.55(\mathrm{~d}, J=6.4 \mathrm{~Hz}, 1 \mathrm{H}, \mathrm{H}-3), 3.96$ (br s, $1 \mathrm{H},-\mathrm{OH}), 3.80(\mathrm{dd}, J=3.1,11.8$ Hz, 1H, H-5), 3.74 (br s, 1H, -OH), 3.61 (dd, $J=4.6,11.8$ Hz, 1H, H-5'), 3.53 (ddd, $J=2.2,5.2$, $7.3 \mathrm{~Hz}, 1 \mathrm{H}, \mathrm{H}-4), 2.41$ (s, 3H, Ar-Me); $\delta_{\mathrm{C}}\left(100 \mathrm{MHz}, \mathrm{MeCN}-\mathrm{d}_{3}\right) 198.62$ (C-6), 173.05 (C-1), 146.21, 131.57, 130.75, 130.24 (ArC), 83.75 (C-2), 72.94 (C-3), 62.59 (C-4), 61.13 (C-5), 21.76 $(\mathrm{Ar}-\underline{\mathrm{Me}}) ; m / z(\mathrm{ESI}+) 288.18\left([\mathrm{M}+\mathrm{Na}]^{+}, 85 \%\right) ;$ HRMS (ESI+) calculated for $\mathrm{C}_{13} \mathrm{H}_{15} \mathrm{NNaO}_{5}$ $\left([\mathrm{M}+\mathrm{Na}]^{+}\right)$288.0842, found 288.0843.

$(2 S, \quad 3 R, \quad 4 R)$-2,3-Dihydroxy-4-hydroxymethyl-2-(4'-methoxyphenylcarbonyl)-1-oxopyrrolidinone, 22b

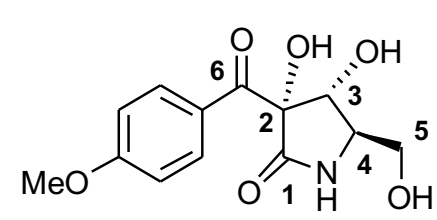

Scale of reaction: $48 \mathrm{mg}(0.0985 \mathrm{mmol})$, product: $15 \mathrm{mg}, 52 \%$; $\mathrm{R}_{f}=$ $0.14($ EtOAc $) ;$ white solid, $\mathrm{mp}=145-147^{\circ} \mathrm{C} ;[\alpha]_{\mathrm{D}}^{23}=-27.3(c=1.21 \mathrm{in}$ 
$\mathrm{MeOH}) ; v_{\max } / \mathrm{cm}^{-1}$ (film) 3304 (br, -NH-), 1688 (s, C=O), 1677 (s, C=O); $\delta_{\mathrm{H}}\left(400 \mathrm{MHz}, \mathrm{MeCN}-\mathrm{d}_{3}\right)$ $8.03(\mathrm{~d}, J=9.0 \mathrm{~Hz}, 2 \mathrm{H}, \mathrm{ArH}), 7.01$ (d, $J=9.0 \mathrm{~Hz}, 2 \mathrm{H}, \mathrm{ArH}), 6.81$ (br s, 1H, -NH-), 4.55 (d, $J=6.4$ Hz, 1H, H-3), 3.87 (s, 3H, -OMe), 3.81 (dd, J=3.1, $11.8 \mathrm{~Hz}, 1 \mathrm{H}, \mathrm{H}-5), 3.62$ (dd, $J=4.6,11.8 \mathrm{~Hz}$, 1H, H-5'), 3.51-3.57 (m, 1H, H-4); $\delta_{\mathrm{C}}\left(100 \mathrm{MHz}, \mathrm{MeCN}^{-d_{3}}\right) 197.10$ (C-6), 173.28 (C-1), 165.27, 133.23, 126.67, 114.89 (ArC), 83.47 (C-2), 73.06 (C-3), 62.67 (C-4), 61.14 (C-5), 56.47 (-OMe); $m / z(\mathrm{ESI}+) 304.21\left([\mathrm{M}+\mathrm{Na}]^{+}, 100 \%\right)$; HRMS $(\mathrm{ESI}+)$ calculated for $\mathrm{C}_{13} \mathrm{H}_{15} \mathrm{NNaO}_{6}\left([\mathrm{M}+\mathrm{Na}]^{+}\right)$ 304.0792, found 304.0800.

(2S, 3R, 4R)-2,3-Dihydroxy-4-hydroxymethyl-2-(octylcarbonyl)-1-oxo-pyrrolidinone, 22c

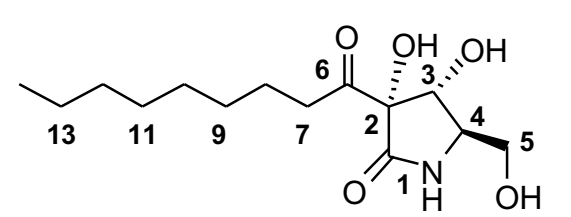

d.r. $\sim 1: 0.2$ about diol

Scale of reaction: $42 \mathrm{mg}(0.851 \mathrm{mmol})$, product: $10 \mathrm{mg}, 41 \%$; $\mathrm{R}_{f}$ $=0.18$ (EtOAc); colourless oil; $v_{\max } / \mathrm{cm}^{-1}$ (film) 3317 (br, $\left.-\mathrm{OH}\right)$, $1691(\mathrm{~s}, \mathrm{C}=\mathrm{O})$; Major diastereomer $\delta_{\mathrm{H}}\left(400 \mathrm{MHz}, \mathrm{MeCN}_{-} \mathrm{d}_{3}\right) 6.61$ (br s, 1H, -NH-), 4.29 (d, $J=6.1 \mathrm{~Hz}, 1 \mathrm{H}, \mathrm{H}-3), 3.76$ (dd, $J=3.5,11.6 \mathrm{~Hz}, 1 \mathrm{H}, \mathrm{H}-5), 3.56$ (dd, $J=$ 5.5, 11.6 Hz, 1H, H-5'), 3.43 (dt, $J=3.5,5.8 \mathrm{~Hz}, 1 \mathrm{H}, \mathrm{H}-4), 2.53-2.72$ (m, 2H, H-7), 1.48-1.60 (m, 2H, H-8), 1.21-1.36 (m, 10H, H-9, H-10, H-11, H-12, H-13), 0.88 (t, J= 7.3 Hz, 3H, H-14); $\delta_{\mathrm{C}}(100$ MHz, MeCN-d 3 ) 210.78 (C-6), 172.44 (C-1), 84.06 (C-2), 71.64 (C-3), 61.96 (C-5), 61.93 (C-4), 38.72 (C-7), 32.59, 30.11, 29.91, 29.80, 23.92 (C-8), 23.38 (C-9, C-10, C-11, C-12, C-13), 14.39 $(\mathrm{C}-14) ; m / z(\mathrm{ESI}+) 288.37\left([\mathrm{M}+\mathrm{H}]^{+}, 35 \%\right)$; HRMS $(\mathrm{ESI}+)$ calculated for $\mathrm{C}_{14} \mathrm{H}_{25} \mathrm{NNaO}_{5}\left([\mathrm{M}+\mathrm{Na}]^{+}\right)$ 310.1625 , found 310.1635 .

(2S, 3R, 4R)-2-(Butylcarbonyl)-2,3-dihydroxy-4-hydroxymethyl-1-oxo-pyrrolidinone, 22d

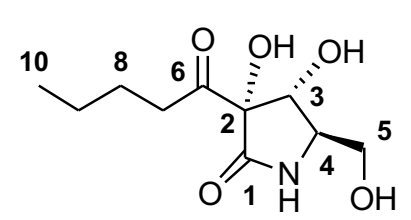

Scale of reaction: $30 \mathrm{mg}(0.0686 \mathrm{mmol})$, product: $9 \mathrm{mg}, 58 \% ; \mathrm{R}_{f}=0.20$

(EtOAc); colourless oil; $[\alpha]_{\mathrm{D}}^{23}=+40.1(c=0.67$ in $\mathrm{MeOH}) ; v_{\max } / \mathrm{cm}^{-1}$

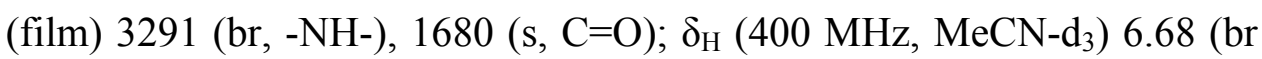
s, 1H, -NH-), 4.30 (d, $J=6.1 \mathrm{~Hz}, 1 \mathrm{H}, \mathrm{H}-3), 3.76$ (dd, $J=3.5,11.6 \mathrm{~Hz}, 1 \mathrm{H}, \mathrm{H}-5), 3.57$ (dd, $J=5.6$, 11.6 Hz, 1H, H-5'), 3.45 (dt, $J=3.5,5.8 \mathrm{~Hz}, 1 \mathrm{H}, \mathrm{H}-4), 2.54-2.76$ (m, 2H, H-7, partially obscured by broad water signal), 1.48-1.59 (m, 2H, H-8), 1.26-1.37 (m, 2H, H-9), 0.90 (t, J= 7.3 Hz, 3H, H- 
10); $\delta_{\mathrm{C}}\left(100 \mathrm{MHz}, \mathrm{MeCN}-\mathrm{d}_{3}\right) 210.76$ (C-6), 172.56 (C-1), 84.10 (C-2), 71.64 (C-3), 62.01 (C-4), 61.96 (C-5), 38.44 (C-7), 26.03 (C-8), 22.90 (C-9), $14.15(\mathrm{C}-10) ; \quad m / z(\mathrm{ESI}+) 254.21\left([\mathrm{M}+\mathrm{Na}]^{+}\right.$, 90\%); HRMS (ESI+) calculated for $\mathrm{C}_{10} \mathrm{H}_{17} \mathrm{NNaO}_{5}\left([\mathrm{M}+\mathrm{Na}]^{+}\right)$254.0999, found 254.1010.

\section{(2S, 3R, 4R)-2,3-Dihydroxy-4-hydroxymethyl-2-(isobutylcarbonyl)-1-oxo-pyrrolidinone, 22e}

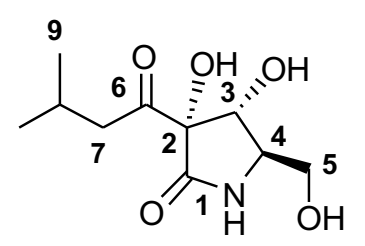

Scale of reaction: $37 \mathrm{mg}(0.0846 \mathrm{mmol})$, product: $6 \mathrm{mg}, 31 \% ; \mathrm{R}_{f}=0.18$

(EtOAc); colourless oil; $[\alpha]_{\mathrm{D}}^{23}=+45.5(c=0.8$ in $\mathrm{MeOH}) ; v_{\max } / \mathrm{cm}^{-1}($ film $)$ $3355(\mathrm{br},-\mathrm{OH}), 1680(\mathrm{~s}, \mathrm{C}=\mathrm{O})$; $\delta_{\mathrm{H}}\left(400 \mathrm{MHz}, \mathrm{MeCN}_{-} \mathrm{d}_{3}\right) 6.68$ (br s, $1 \mathrm{H},-$ NH-), 4.29 (d, $J=6.0$ Hz, 1H, H-3), 3.77 (dd, $J=3.6,11.6$ Hz, 1H, H-5), 3.57 (dd, $J=5.6,11.7$ Hz, $1 \mathrm{H}, \mathrm{H}-5$ '), 3.44 (dt, $J=3.5,5.8 \mathrm{~Hz}, 1 \mathrm{H}, \mathrm{H}-4), 2.59$ (dd, $J=6.8,17.8 \mathrm{~Hz}, 1 \mathrm{H}, \mathrm{H}-7), 2.48(\mathrm{dd}, J=$ 6.7, $\left.17.8 \mathrm{~Hz}, 1 \mathrm{H}, \mathrm{H}-7^{\prime}\right), 2.09-2.19(\mathrm{~m}, 1 \mathrm{H}, \mathrm{H}-8), 0.91$ (d, J=6.7 Hz, 6H, H-9); $\delta_{\mathrm{C}}(100 \mathrm{MHz}$, MeCN-d 3 ) 210.23 (C-6), 172.49 (C-1), 84.09 (C-2), 71.52 (C-3), 62.02 (C-4), 61.97 (C-5), 47.39 (C-7), $24.61(\mathrm{C}-8), 22.72(\mathrm{C}-9) ; m / z(\mathrm{ESI}+) 232.23\left([\mathrm{M}+\mathrm{H}]^{+}, 20 \%\right)$; HRMS (ESI+) calculated for $\mathrm{C}_{10} \mathrm{H}_{17} \mathrm{NNaO}_{5}\left([\mathrm{M}+\mathrm{Na}]^{+}\right)$254.0999, found 254.1003.

\section{(2S, 3R, 4R)-2,3-Dihydroxy-4-hydroxymethyl-2-(phenylacetylenecarbonyl)-1-oxo-} pyrrolidinone, $22 \mathrm{f}$

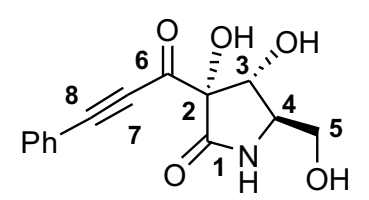

Scale of reaction: $70 \mathrm{mg}(0.145 \mathrm{mmol})$, product: $19 \mathrm{mg}, 48 \% ; \mathrm{R}_{f}=0.17$ (EtOAc); white solid, $\mathrm{mp}=149-150^{\circ} \mathrm{C} ; v_{\max } / \mathrm{cm}^{-1}$ (film) 3299 (br, -OH), d.r. $\sim$ 1:0.3 about diol 2193 (s, alkyne), 1699 (s, C=O), $1674(\mathrm{~s}, \mathrm{C}=\mathrm{O})$; Major diastereomer: $\delta_{\mathrm{H}}(400$ MHz, MeCN-d $\mathrm{d}_{3}$ 7.63-7.71 (m, 2H, PhH), 7.54-7.62 (m, 1H, PhH), 7.44-7.52 (m, 2H, PhH), 6.69 (br s, 1H, -NH-), 4.74 (d, $J=6.7 \mathrm{~Hz}, 1 \mathrm{H}, \mathrm{H}-3), 3.84$ (dd, $J=3.0,11.9 \mathrm{~Hz}, 1 \mathrm{H}, \mathrm{H}-5), 3.64$ (dd, $J=$ 4.8, $11.9 \mathrm{~Hz}, 1 \mathrm{H}, \mathrm{H}-5$ ') $3.50(\mathrm{ddd}, J=3.0,4.8,6.7 \mathrm{~Hz}, 1 \mathrm{H}, \mathrm{H}-4) ; \delta_{\mathrm{C}}\left(100 \mathrm{MHz}, \mathrm{MeCN}-\mathrm{d}_{3}\right) 186.86$ (C-6), 171.34 (C-1), 134.34, 132.73, 130.00, 119.95 (PhC), 97.73 (C-8), 86.09 (C-2), 85.87 (C-7), $72.16(\mathrm{C}-3), 61.71(\mathrm{C}-4), 61.30(\mathrm{C}-5) ; \mathrm{m} / z(\mathrm{ESI}+) 276.48\left([\mathrm{M}+\mathrm{H}]^{+}, 35 \%\right)$; HRMS (ESI+) calculated for $\mathrm{C}_{14} \mathrm{H}_{13} \mathrm{NNaO}_{5}\left([\mathrm{M}+\mathrm{Na}]^{+}\right)$298.0686, found 298.0698. 
(2S, 3R, 4R)-2,3-Dihydroxy-4-hydroxymethyl-2-(butylacetylenecarbonyl)-1-oxo-pyrrolidinone, $22 \mathrm{~g}$

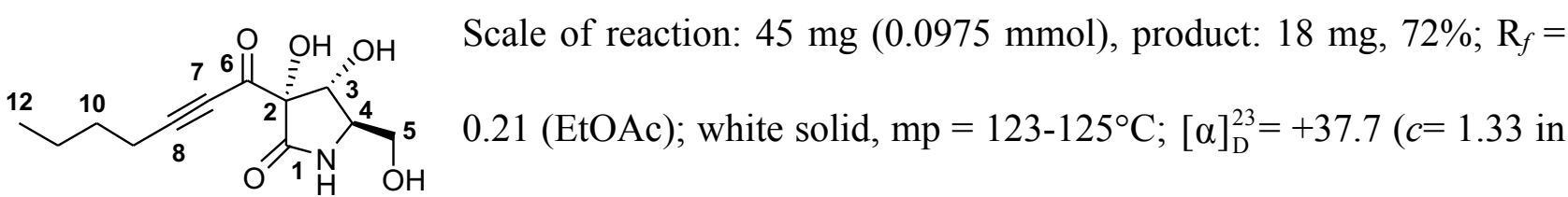
$\mathrm{MeOH}) ; v_{\max } / \mathrm{cm}^{-1}$ (film) 3356 (br, -NH-), 2211 (s, alkyne), 1701 (s, C=O), 1682 (s, C=O); $\delta_{\mathrm{H}}(400$ MHz, MeCN-d $\left.{ }_{3}\right) 6.67$ (br s, $\left.1 \mathrm{H},-\mathrm{NH}-\right), 4.57(\mathrm{~d}, J=6.8 \mathrm{~Hz}, 1 \mathrm{H}, \mathrm{H}-3), 3.81(\mathrm{dd}, J=3.2,11.7 \mathrm{~Hz}$, 1H, H-5), 3.57 (dd, $J=5.5,11.7 \mathrm{~Hz}, 1 \mathrm{H}, \mathrm{H}-5$ '), 3.46 (ddd, $J=3.1,5.5,6.8 \mathrm{~Hz}, 1 \mathrm{H}, \mathrm{H}-4), 2.47$ (t, $J$ $=7.0 \mathrm{~Hz}, 2 \mathrm{H}, \mathrm{H}-9)$ (overlapping broad water signal at baseline), 1.51-1.63 (m, 2H, H-10), 1.36-1.49 (m, 2H, H-11), 0.92 (t, $J=7.3 \mathrm{~Hz}, 3 \mathrm{H}, \mathrm{H}-12) ; \delta_{\mathrm{C}}\left(100 \mathrm{MHz}, \mathrm{MeCN}-\mathrm{d}_{3}\right) 186.67$ (C-6), $171.36(\mathrm{C}-1)$, 103.00 (C-8), 85.69 (C-2), 78.77 (C-7), 72.39 (C-3), 61.85 (C-5), 61.79 (C-4), 30.27 (C-10), 22.66 (C-11), $19.38(\mathrm{C}-9), 13.77(\mathrm{C}-12) ; \mathrm{m} / z(\mathrm{ESI}+) 256.26\left([\mathrm{M}+\mathrm{H}]^{+}, 15 \%\right)$; HRMS (ESI+) calculated for $\mathrm{C}_{12} \mathrm{H}_{18} \mathrm{NO}_{5}\left([\mathrm{M}+\mathrm{H}]^{+}\right)$256.1179, found 256.1175.

\section{General procedure for partial reductive hydrogenation-isomerisation of ynones $8 \mathrm{f}, \mathrm{g}$ and $22 \mathrm{f}, \mathrm{g}$}

To an EtOAc solution (approx. 0.02-0.05 M) of the ynone (between 0.07-0.1 mmol scale) was added Lindlar's catalyst $\left(5 \% \mathrm{Pd} / \mathrm{CaCO}_{3}\right.$ (poisoned with $\left.\mathrm{Pb}\right), 10-15 \%$ wt. of ynone). Quinoline (1 $\mu \mathrm{L}$ ) was added for the reduction of all butylethynyl derivatives. The suspension was stirred at r.t. with $\mathrm{H}_{2}$ gas (balloon) bubbled through the mixture and the progress of the reaction was monitored using LRMS analysis. The suspension was then filtered over Celite, rinsed with EtOAc and the solvent removed in vacuo to give the crude product. The crude from the above reduction was dissolved in EtOAc (approx. $0.02 \mathrm{M}$ ), with one drop of dilute $\mathrm{I}_{2} /$ EtOAc solution added. The solution irradiated using a 500W halogen lamp with stirring for 5-6 h. The reaction was quenched with aq. $\mathrm{Na}_{2} \mathrm{~S}_{2} \mathrm{O}_{3}$ solution and extracted with EtOAc. The combined organic layers was dried with anhydrous $\mathrm{MgSO}_{4}$, filtered and the solvents removed in vacuo to give the crude. Purification of the crude was done via reverse-phase preparative HPLC on an Agilent 1200 series system with 
Phenomenex ${ }^{\circledR} \quad$ Luna $\mathrm{C} 18(2)$ column $(10.0 \times 100 \mathrm{~mm}, 5 \mu \mathrm{m})$ using the elution gradient with a $\mathrm{MeCN}-\mathrm{H}_{2} \mathrm{O}$ mobile phase at a flow rate of $5 \mathrm{~mL} / \mathrm{min}$ and detection at $\lambda 210$ and $254 \mathrm{~nm}$.

$(2 S, \quad 4 R)-2-H y d r o x y-4-h y d r o x y m e t h y l-2-[(E)-p h e n y l v i n y l e n e c a r b o n y l]-1-o x o-p y r r o l i d i n o n e$, $(E)-23 a$

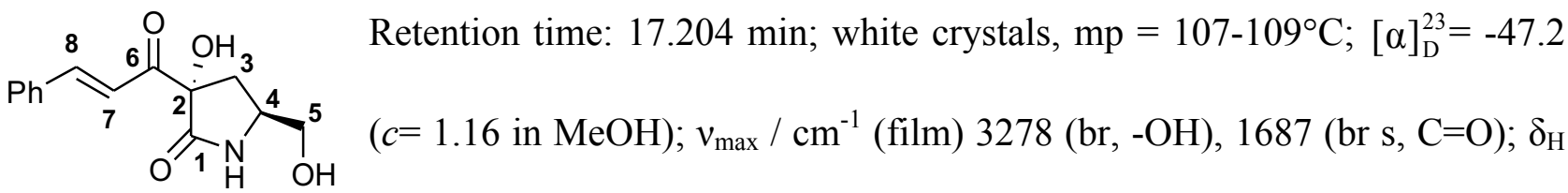
$\left(400 \mathrm{MHz}, \mathrm{MeCN}_{-} \mathrm{d}_{3}\right) 7.77$ (d, $\left.J=15.9 \mathrm{~Hz}, 1 \mathrm{H}, \mathrm{H}-8\right)$, 7.64-7.71 (m, 2H, PhH), 7.39-7.51 (m, 3H, PhH), 7.32 (d, $J=15.9$ Hz, 1H, H-7), 6.84 (br s , 1H, -NH-), 4.75 (br s , 1H, -OH), 3.86-3.91 (m, 1H, H-4), 3.69 (dd, $J=3.8,11.4 \mathrm{~Hz}, 1 \mathrm{H}, \mathrm{H}-5), 3.51(\mathrm{dd}, J=5.5,11.4 \mathrm{~Hz}, 1 \mathrm{H}, \mathrm{H}-5$ '), 2.50 (dd, $J=$ 6.1, 14.4 Hz, 1H, H-3), 2.14 (dd, $J=7.8,14.4 \mathrm{~Hz}, 1 \mathrm{H}, \mathrm{H}-3$ '); $\delta_{\mathrm{C}}\left(100 \mathrm{MHz}, \mathrm{MeCN}_{-} \mathrm{d}_{3}\right) 198.60(\mathrm{C}-$ 6), 174.84 (C-1), 145.48 (C-8), 135.46, 132.02, 130.06, 129.78 (PhC), 121.15 (C-7), 83.70 (C-2), 64.68 (C-5), 53.96 (C-4), 35.59 (C-3); m/z (ESI+) $262.53\left([\mathrm{M}+\mathrm{H}]^{+}, 98 \%\right)$; HRMS (ESI+) calculated for $\mathrm{C}_{14} \mathrm{H}_{16} \mathrm{NO}_{4}\left([\mathrm{M}+\mathrm{H}]^{+}\right)$262.1074, found 262.1076.

$(2 S, \quad 4 R)-2-H y d r o x y-4-h y d r o x y m e t h y l-2-[(E)$-butylvinylenecarbonyl]-1-oxo-pyrrolidinone, $(E)-23 b$

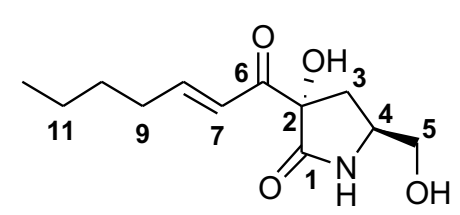

Retention time: $17.98 \mathrm{~min}$; colourless oil; $[\alpha]_{\mathrm{D}}^{23}=+45.3(c=1.07$ in $\mathrm{CH}_{2} \mathrm{Cl}_{2}$ ); $v_{\max } / \mathrm{cm}^{-1}$ (film) 3303 (br, -OH), 1702 (br s, C=O; with shoulder towards smaller wavenumber); $\delta_{\mathrm{H}}\left(400 \mathrm{MHz}, \mathrm{MeCN}-\mathrm{d}_{3}\right) 7.07$ (dt, $J=7.0,15.5 \mathrm{~Hz}, 1 \mathrm{H}, \mathrm{H}-8), 6.76$ (br s , 1H, -NH-), 6.56 (dt, $J=1.6,15.5 \mathrm{~Hz}, 1 \mathrm{H}, \mathrm{H}-7$ ), 4.60 (br s, 1H, -OH), 3.79-3.90 (m, 1H, H-4), 3.64 (dd, $J=3.9,11.3 \mathrm{~Hz}, 1 \mathrm{H}, \mathrm{H}-5), 3.45$ (dd, $J=5.9,11.3$ Hz, 1H, H-5'), 2.35 (dd, $J=6.4,14.3 \mathrm{~Hz}, 1 \mathrm{H}, \mathrm{H}-3$ ), 2.24-2.29 (m, 2H, H-9) (partially obscured by water signal), 2.06 (dd, $J=7.6,14.3 \mathrm{~Hz}, 1 \mathrm{H}, \mathrm{H}-3$ '), $1.41-1.51$ (m, 2H, H-10), 1.28-1.40 (m, 2H, H11), $0.91(\mathrm{t}, J=7.3 \mathrm{~Hz}, 3 \mathrm{H}, \mathrm{H}-12) ; \delta_{\mathrm{C}}\left(100 \mathrm{MHz}, \mathrm{MeCN}_{-} \mathrm{d}_{3}\right) 198.28$ (C-6), 174.80 (C-1), 151.75 (C-8), 124.49 (C-7), 83.30 (C-2), 64.82 (C-5), 54.03 (C-4), 35.73 (C-3), 32.98 (C-9), 30.78 (C-10), 
$23.01(\mathrm{C}-11), 14.12(\mathrm{C}-12) ; \mathrm{m} / z(\mathrm{ESI}+) 264.58\left([\mathrm{M}+\mathrm{Na}]^{+}, 100 \%\right)$; HRMS (ESI+) calculated for $\mathrm{C}_{12} \mathrm{H}_{19} \mathrm{NNaO}_{4}\left([\mathrm{M}+\mathrm{Na}]^{+}\right)$264.1206, found 264.1206.

(2S, 4R)-2-(Hexylcarbonyl)-2-hydroxy-4-hydroxymethyl-1-oxo-pyrrolidinone, 23c

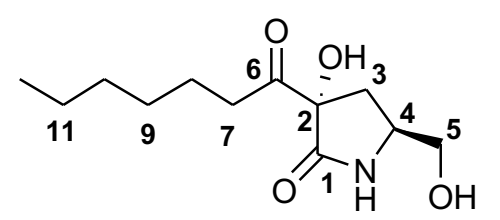

Retention time: $22.20 \mathrm{~min}$; colourless oil; $[\alpha]_{\mathrm{D}}^{23}=+75.0(c=0.84$ in $\mathrm{CH}_{2} \mathrm{Cl}_{2}$ ); $v_{\max } / \mathrm{cm}^{-1}$ (film) 3289 (br, -OH), 1715 (br s, $\left.\mathrm{C}=\mathrm{O}\right), 1690$ (br s, C=O); $\delta_{\mathrm{H}}\left(400 \mathrm{MHz}, \mathrm{MeCN}_{-} \mathrm{d}_{3}\right) 6.67$ (br s, 1H, -NH-), 4.47 (br s, 1H, -OH), 3.74-3.87 (m, 1H, H-4), 3.61 (dd, $J=4.2,11.2 \mathrm{~Hz}, 1 \mathrm{H}, \mathrm{H}-5), 3.44$ (dd, $J=6.3,11.2$ Hz, 1H, H-5'), 2.68 (dd, J=7.3, 17.9 Hz, 1H, H-7), 2.57 (dd, J= 7.3, 17.9 Hz, 1H, H-7'), 2.32 (dd, $J=6.3,14.2 \mathrm{~Hz}, 1 \mathrm{H}, \mathrm{H}-3), 2.04$ (dd, $J=7.6,14.2 \mathrm{~Hz}, 1 \mathrm{H}, \mathrm{H}-3$ '), 1.47-1.60 (m, 2H), 1.26-1.32 (m, 6H) (H-8, H-9, H-10, H-11), 0.88 (t, $J=7.3 \mathrm{~Hz}, 3 \mathrm{H}, \mathrm{H}-12)$; $\delta_{\mathrm{C}}\left(100 \mathrm{MHz}, \mathrm{MeCN}-\mathrm{d}_{3}\right) 211.01$ (C-6), 174.76 (C-1), 84.05 (C-2), 65.09 (C-5), 53.90 (C-4), 37.96 (C-7), 35.67 (C-3), 32.36, 29.48, 24.17, 23.25 (C-8, C-9, C-10, C-11), 14.35 (C-12); m/z (ESI+) $244.58\left([\mathrm{M}+\mathrm{H}]^{+}, 85 \%\right)$; HRMS (ESI+) calculated for $\mathrm{C}_{12} \mathrm{H}_{22} \mathrm{NO}_{4}\left([\mathrm{M}+\mathrm{H}]^{+}\right)$244.1543, found 244.1544 .

$(2 S, \quad 3 R, \quad 4 R)-2,3-D i h y d r o x y-4-h y d r o x y m e t h y l-2-[(E)$-phenylvinylenecarbonyl]-1-oxopyrrolidinone, $(E)-23 d$

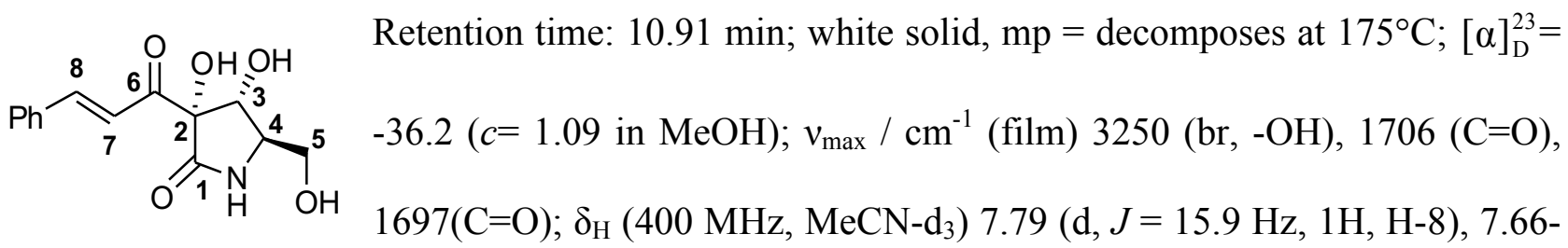
7.73 (m, 2H, PhH), 7.40-7.50 (m, 3H, PhH), 7.23 (d, J=15.9 Hz, 1H, H-7), 6.63 (br s, 1H, -NH-), $4.41(\mathrm{~d}, J=6.2 \mathrm{~Hz}, 1 \mathrm{H}, \mathrm{H}-3), 3.81(\mathrm{dd}, J=3.4,11.7 \mathrm{~Hz}, 1 \mathrm{H}, \mathrm{H}-5), 3.6281(\mathrm{dd}, J=5.4,11.6 \mathrm{~Hz}$, $1 \mathrm{H}, \mathrm{H}-5$ '), 3.50 (ddd, $J=3.4,5.4,6.2 \mathrm{~Hz}, 1 \mathrm{H}, \mathrm{H}-4) ; \delta_{\mathrm{C}}\left(100 \mathrm{MHz}, \mathrm{MeCN}-\mathrm{d}_{3}\right) 198.28(\mathrm{C}-6), 172.3$ (C-1), 145.58 (C-8), 135.41, 132.10, 130.07, 129.82 (PhC), 121.17 (C-7), 83.73 (C-2), 71.89 (C-3), $62.22(\mathrm{C}-4), 62.11(\mathrm{C}-5) ; \mathrm{m} / \mathrm{z}(\mathrm{ESI}+) 278.65\left([\mathrm{M}+\mathrm{H}]^{+}, 100 \%\right) ;$ HRMS (ESI+) calculated for $\mathrm{C}_{14} \mathrm{H}_{16} \mathrm{NO}_{5}\left([\mathrm{M}+\mathrm{H}]^{+}\right)$278.1023, found 278.1024. 
$(2 S$, $3 R, \quad 4 R)-2,3-D i h y d r o x y-4-h y d r o x y m e t h y l-2-[(E)$-butylvinylenecarbonyl]-1-oxo-

pyrrolidinone, $(E)-23 e$

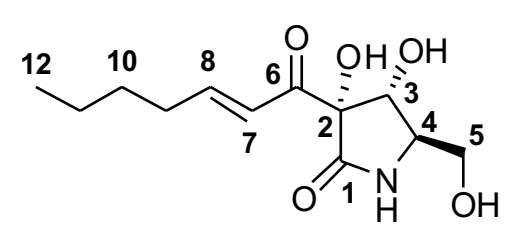

Retention time: $15.31 \mathrm{~min}$; colourless oil; $[\alpha]_{\mathrm{D}}^{23}=+37.2(c=0.68$ in $\mathrm{MeOH}) ; v_{\max } / \mathrm{cm}^{-1}$ (film) 3346 (br, -OH), 1701 (br s, C=O with shoulder towards smaller wavenumber); $\delta_{\mathrm{H}}\left(400 \mathrm{MHz}, \mathrm{MeCN}-\mathrm{d}_{3}\right)$ 7.09 (dt, $J=7.0,15.5 \mathrm{~Hz}, 1 \mathrm{H}, \mathrm{H}-8), 6.54$ (br s, 1H, -NH-), 6.50 (dt, $J=1.6,15.6 \mathrm{~Hz}, 1 \mathrm{H}, \mathrm{H}-7$ ), 4.54 (s, 1H, -OH C2), 4.31 (dd, $J=6.3,8.6 \mathrm{~Hz}, 1 \mathrm{H}, \mathrm{H}-3), 3.73-3.83(\mathrm{~m}, 1 \mathrm{H}, \mathrm{H}-5), 3.58$ (ddd, $J=5.1$, 6.6, 11.7 Hz, 1H, H-5'), 3.51 (d, $J=8.6 \mathrm{~Hz}, 1 \mathrm{H},-\mathrm{OH} \mathrm{C} 3), 3.42-3.48$ (m, 1H, H-4), 3.05 (dd, $J=$ 5.1, 6.6 Hz, 1H, -OH C5), 2.27 (apparent dq, $J=1.6,7.1 \mathrm{~Hz}, 2 \mathrm{H}, \mathrm{H}-9$ ), 1.41-1.55 (m, 2H, H-10), 1.28-1.41 (m, 2H, H-11), $0.91(\mathrm{t}, J=7.3 \mathrm{~Hz}, 3 \mathrm{H}, \mathrm{H}-12) ; \delta_{\mathrm{C}}\left(100 \mathrm{MHz}, \mathrm{MeCN}_{-} \mathrm{d}_{3}\right) 197.71(\mathrm{C}-6)$, 172.24 (C-1), 152.07 (C-8), 124.62 (C-7), 83.33 (C-2), 71.77 (C-3), 62.20 (C-4), 61.87 (C-5), 33.04 (C-9), 30.79 (C-10), 23.02 (C-11), $14.11(\mathrm{C}-12) ; \mathrm{m} / z$ (ESI+) $258.86\left([\mathrm{M}+\mathrm{H}]^{+}, 75 \%\right)$; HRMS (ESI+) calculated for $\mathrm{C}_{12} \mathrm{H}_{20} \mathrm{NO}_{5}\left([\mathrm{M}+\mathrm{H}]^{+}\right)$258.1336, found 258.1334.

\section{Acknowledgements}

S.W.B.T. gratefully acknowledges the award of an A*STAR Graduate Scholarship. We thank Wendy Sobey for preparation of the agar plates for the bioassays, Tharindi Panduwawala for assistance with the biological testing of some of the compounds, and Srinivasulu Aitipamula (ICES) for the X-ray crystallographic analysis of compounds $\mathbf{1 1}$ and 22b.

Electronic Supplementary Supporting Information. ${ }^{1} \mathrm{H}$ and ${ }^{13} \mathrm{C}$ NMR spectra of all compounds.

\section{References}

1. D. J. Newman and G. M. Cragg, J. Nat. Prod., 2012, 75, 311-335.

2. D. J. Newman, J. Med. Chem., 2008, 51, 2589-2599.

3. D. J. Newman and G. M. Cragg, J. Nat. Prod., 2007, 70, 461-477.

4. D. J. Newman, G. M. Cragg and K. M. Snader, J. Nat. Prod., 2003, 66, 1022 -1037. 
5. H. W. Boucher, G. H. Talbot, D. K. Benjamin, J. Bradley, R. J. Guidos, R. N. Jones, B. E. Murray, R. A. Bonomo and D. Gilbert, Clin. Infect. Dis., 2013, 56, 1685-1694.

6. M. S. Butler and M. A. Cooper, J. Antibiot., 2011, 64, 413-425.

7. M. Saleem, M. Nazir, M. S. Ali, H. Hussain, Y. S. Lee, N. Riaza and A. Jabbara, Nat. Prod. Rep., 2010, 27, 238-254.

8. S. J. Projan and P. A. Bradford, Current Opinion in Microbiology, 2007, 10, 441-446.

9. UK Five Year Antimicrobial Resistance Strategy 2013-2018, Department for Health and Department for Environment, Food and Rural Affairs, 2013.

10. Antimicrobial Resistance. Global Report on Surveillance, World Health Organization, 2014.

11. R. Wise and L. J. V. Piddock, The Urgent Need Regenerating antibacterial drug discovery development, British Society for Antimicrobial Chemotherapy, 2010.

12. B. Spellberg, R. Guidos, D. Gilbert, J. Bradley, H. W. Boucher, W. M. Scheld, J. G. Bartlett and J. J. Edwards, Clinical Infectious Diseases, 2008, 46, 155-164.

13. G. H. Talbot, J. Bradley, J. J.E. Edwards, D. Gilbert, M. Scheld and J. G. Bartlett, Clinical Infectious Diseases, 2006, 42, 657-668.

14. F. Fasehun, Bulletin of the World Health Organization, 1999, 77(3), 211.

15. M. S. Kinch, E. Patridge, M. Plummer and D. Hoyer, Drug Discovery Today, 2014, 19, 1283-1287.

16. J. O'Neill, Antimicrobial Resistance:Tackling a crisis for the health and wealth of nations, 2014.

17. S. B. Singh, Biorg. Med. Chem. Lett., 2014, 24, 3683-3689.

18. L. L. Silver, Clin. Microbiol. Rev., 2011, 24, 71-109.

19. T. Dick and D. Young, Future Microbiol., 2011, 6, 603-604.

20. M. N. Gwynn, A. Portnoy, S. F. Rittenhouse and D. J. Payne, Ann. N.Y. Acad. Sci. , 2010, 1213, 5-19.

21. K. J. Williams and R. P. Bax, Curr. Opin. Invest. Drugs, 2009, 10, 157-163.

22. D. J. Payne, M. N. Gwynn, D. J. Holmes and D. L. Pompliano, Nat. Rev. Drug Discovery, 2007, 6, 29-40.

23. P. Fernandes, Nat Biotech, 2006, 24, 1497-1503.

24. S. J. Projan, Current Opinion in Pharmacology, 2002, 2, 513-522.

25. S. J. Projan, Current Opinion in Pharmacology, 2003, 3, 457-458.

26. S. Tuske, S. G. Sarafianos, X. Wang, B. Hudson, E. Sineva, J. Mukhopadhyay, J. J. Birktoft, O. Leroy, S. Ismail, A. D. Clark, C. Dharia, A. Napoli, O. Laptenko, J. Lee, S. Borukhov, R. H. Ebright and E. Arnold, Cell, 2005, 122, 541-552.

27. J. Phillips, M. Goetz, S. Smith, D. Zink, J. Polishook, R. Onishi, S. S, J. Wiltsie, J. Allocco, J. Sigmund, K. Dorso, S. Lee, S. Skwish, M. de la Cruz, J. Martín, F. Vicente, O. Genilloud, J. Lu, R. Painter, K. Young, K. Overbye, R. Donald and S. Singh, Chem Biol., 2011, 18, 955965.

28. Y.-C. Jeong and M. G. Moloney, Molecules, 2015, 20, 3582-3627

29. Y.-C. Jeong, M. Anwar, M. G. Moloney, Z. Bikadi and E. Hazai, Chem. Sci., 2013, 4, 10081015.

30. Y.-C. Jeong and M. G. Moloney, Beilstein J. Org. Chem., 2013, 9, 1899-1906.

31. R. E. Schwartz, G. L. Helms, E. A. Bolessa, K. E. Wilson, R. A. Giacobbe, J. S. Tkacz, G. F. Bills, J. M. Liesch, D. L. Zink, J. E. Curotto, B. Pramanik and J. C. Onishi, Tetrahedron, 1994, 50, 1675-1686.

32. C. Bodur, O. Kutuk, G. Karsli-Uzunbas, T. T. Isimjan, P. Harrison and H. Basaga, PLOS One, 2013, 8, e56369.

33. B. S. W. Tan, C. L. L. Chai, M. G. Moloney and A. L. Thompson, J. Org. Chem., 2015, 80, 2661-2675. 
34. M. Bergeron-Brlek, J. Goodwin-Tindall, N. Cekic, C. Roth, W. F. Zandberg, X. Shan, V. Varghese, S. Chan, G. J. Davies, D. J. Vocadlo and R. Britton, Angew. Chem. Int. Ed. Engl., 2015, 54, 15429-15433.

35. J. Christoffers, T. Werner, S. Unger and W. Frey, Eur. J. Org. Chem., 2003, 425-431.

36. M. J. Beard, J. H. Bailey, D. T. Cherry, M. G. Moloney, S. B. Shim, K. Statham, M. Bamford and R. B. Lamont, Tetrahedron, 1996, 52, 3719-3740 and corrigenda 1997, 3753, 1177.

37. Y.-C. Jeong and M. G. Moloney, J. Org. Chem., 2011, 76, 1342-1354.

38. I. F. Cottrell, P. J. Davis and M. G. Moloney, Tetrahedron: Asymmetry, 2004, 15, 12391242.

39. M. J. Houghton, N. A. Biok, C. J. Huck, R. F. Algera, I. Keresztes, S. W. Wright and D. B. Collum, J. Org. Chem., 2016, 81, 4149-4157.

40. J. H. Bailey, A. T. J. Byfield, P. J. Davis, A. C. Foster, M. Leech, M. G. Moloney, M. Muller and C. K. Prout, J. Chem. Soc., Perkin Trans 1, 2000, 1977-1982.

41. M. J. Houghton, C. J. Huck, S. W. Wright and D. B. Collum, J. Am. Chem. Soc., 2016, 138, 10276-10283.

42. S.-J. Han and B. M. Stoltz, Tetrahedron Letters, 2016, 57, 2233-2235.

43. J. H. Bailey, D. Cherry, J. Dyer, M. G. Moloney, M. J. Bamford, S. Keeling and R. B. Lamont, J. Chem. Soc., Perkin Trans. 1, 2000, 2783-2792.

44. P. W. H. Chan, I. F. Cottrell and M. G. Moloney, Tetrahedron Lett., 1997, 38, 5891-5894.

45. T. K. M. Shing, E. K. W. Tam, V. W.-F. Tai, I. H. F. Chung and Q. Jiang, Chem. Eur. J. , 1996, 2, 50-57.

46. Crystallographic data (excluding structure factors) for $\mathbf{1 1}$ and $\mathbf{2 2 b}$ have been deposited with the Cambridge Crystallographic Data Centre (CCDC 1509400 and 1509399 respectively) and copies of these data can be obtained free of charge via www.ccdc.cam.ac.uk/data_request/cif.

47. J. H. Bailey, D. T. Cherry, K. M. Crapnell, M. G. Moloney, S. B. Shim, M. Bamford and R. B. Lamont, Tetrahedron, 1997, 53, 11731-11744.

48. S. W. Wright, C. Choi, S. Chung, B. P. Boscoe, S. E. Drozda, J. J. Mousseau and J. D. Trzupek, Org. Lett., 2015, 17, 5204-5207.

49. M. Smith, D. H. Rammler, I. H. Goldberg and H. G. Khorana, J. Am. Chem. Soc., 1962, 84, 430-440.

50. F. A. Davis, L. C. Vishwakarma, J. M. Billmers and J. Finn, J. Org. Chem., 1984, 49, 32413243.

51. Bioassay of products:65 Microbiological assays were performed by the hole- plate method with the test organism Staphylococcus aureus DS267 or E. coli X580. Solutions $(100 \mu \mathrm{L})$ of the compounds to be tested $(4,2,1,0.5 \mathrm{mg} / \mathrm{mL})$ were loaded into wells in bioassay plates, and incubated overnight at $37^{\circ} \mathrm{C}$. The diameters of the resultant inhibition zones were measured $( \pm 0.5 \mathrm{~mm})$, and relative potency estimated by reference to standards prepared with the positive control, Cephalosporin $\mathrm{C}$; this is expressed as zone diameter per $\mathrm{M}$, of the analyte relative to cephalosporin $\mathrm{C}$ standard.

52. Calculated using Marvin 5.3.8, 2010, ChemAxon (http://www.chemaxon.com).

53. R. O'Shea and H. E. Moser, J. Med. Chem., 2008, 51, 2871-2878.

54. C. Weidenmaier and A. Peschel, Nat. Rev. Microbiol., 2008, 6, 276-287.

55. W. W. Wilson, M. M. Wade, S. C. Holman and F. R. Champlin, J. Microbiol. Methods, 2001, 43, 153-164.

56. C. A. Lipinski, F. Lombardo, B. W. Dominy and P. J. Feeney, Adv. Drug Delivery Rev., 2001, 46, 3-26.

57. C. A. Holloway, C. J. Matthews, M. G. Moloney, C. F. Roberts and M. Yaqoob, Chem. Biol. Drug. Des., 2011, 78, 229-235.

58. Y.-C. Jeong and M. G. Moloney, Synlett., 2009, 2487-2491

59. F. Lovering, MedChemComm, 2014, 4, 515-519. 
60. F. Lovering, J. Bikker and C. Humblet, J. Med. Chem., 2009, 52, 6752-6756.

61. A. Stefanucci, E. Novellino, R. Costante and A. Mollica, Heterocycles, 2014, 89, 1801 1825.

62. S. K. Panday, J. Prasad and D. K. Dikshit, Tetrahedron: Asymmetry, 2009, 20, 1581-1632.

63. C. Najera and M. Yus, Tetrahedron:Asymmetry, 1999, 10, 2245-2303.

64. C. J. Andreas, N. Spetseris, J. R. Norton and A. I. Meyers, Tetrahedron Lett., 1995, 36, 1613-1616.

65. B. Smith, S. C. Warren, G. G. F. Newton and E. P. Abraham, Biochem. J., 1967, 103, 877890. 